\title{
HUMAN KNEE FEA MODEL FOR TRANSTIBIAL AMPUTEE TIBIAL CARTILAGE PRESSURE IN GAIT AND CYCLING
}

\author{
A Thesis \\ presented to \\ the Faculty of California Polytechnic State University, \\ San Luis Obispo
}

\author{
In Partial Fulfillment \\ of the Requirements for the Degree \\ Master of Science in Mechanical Engineering
}

\author{
by \\ Gregory Lane \\ June 2018
}


(C) 2018

Gregory Lane

ALL RIGHTS RESERVED 


\section{COMMITTEE MEMBERSHIP}

TITLE: Human Knee FEA Model for Transtibial Amputee Tibial Cartilage Pressure In Gait and Cycling

AUTHOR: Gregory Lane

DATE SUBMITTED: June 2018

COMMITTEE CHAIR: Scott Hazelwood, Ph.D.

Professor of Biomedical Engineering

COMMITTEE MEMBER: Stephen Klisch, Ph.D.

Professor of Mechanical Engineering

COMMITTEE MEMBER: Joseph Mello, Ph.D.

Professor of Mechanical Engineering 


\section{ABSTRACT}

Human Knee FEA Model for Transtibial Amputee Tibial Cartilage Pressure In Gait and Cycling

$$
\text { Gregory Lane }
$$

Osteoarthritis $(\mathrm{OA})$ is a debilitating disease affecting roughly 31 million Americans. The incidence of $\mathrm{OA}$ is significantly higher for persons who have suffered a transtibial amputation. Abnormal cartilage stress can cause higher OA risk, however it is unknown if there is a connection between exercise type and cartilage stress. To help answer this, a tibiofemoral FEA model was created. Utilizing linear elastic isotropic materials and non-linear springs, the model was validated to experimental cadaveric data. In a previous study, 6 control and 6 amputee subjects underwent gait and cycling experiments. The resultant knee loads were analyzed to find the maximum compressive load and the respective shear forces and rotation moments for each trial, which were then applied to the model. Maximum tibial contact stress values were extracted for both the medial and lateral compartments. Only exercise choice in the lateral compartment was found to be a significant interaction $(p<0.0001)$. No other interactions in either compartment were significant. This suggests that cycling reduces the risk for lateral OA regardless of amputation status and medial OA risk is unaffected. This study also developed a process for creating subject-specific FEA models.

Keywords: Osteoarthritis, human knee, transtibial amputee, finite element analysis, gait, cycling, articular cartilage 


\section{ACKNOWLEDGMENTS}

Thank you to Dr. Scott Hazelwood and Dr. Stephen Klisch for their immeasurable support and guidance over the past few years. Working in the Cal Poly Human Motion Biomechanics Lab has been both immensely enriching and satisfying.

Thank you to Dr. Joseph Mello for all of his instruction throughout my undergraduate career and his review of this thesis.

Thank you to Dr. David Tuttle and Paul Flaherty of French Hospital Medical Center for their help in determination of the correct MRI sequences.

A special thanks to all of the HMB lab personnel for their friendship and assistance.

Thank you to all of my friends and family for their love and support.

Finally, thank you a thousand times to Michael Rumery for his friendship and partnership on this project. Without his tireless efforts, this project would still be in its infancy. Shesh besh.

This work was supported by the Defense Health Program, through the Department of Defense Broad Agency Announcement for Extramural Medical Research Program Number W81XWH-BAA-14-1under Award No. W81XWH-161-0051. Opinions, interpretations, conclusions and recommendations are those

of the author and are not necessarily endorsed by the Department of Defense. 


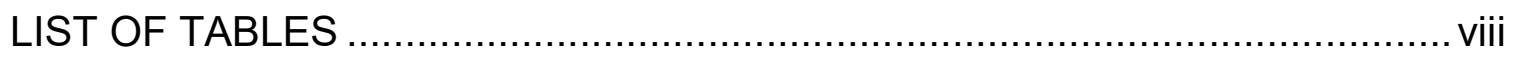

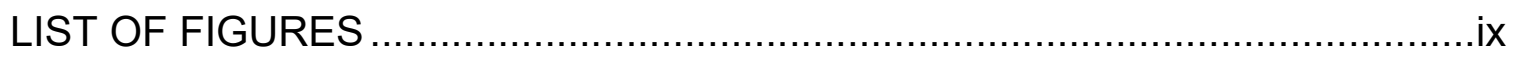

\section{CHAPTER}

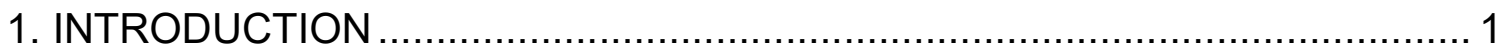

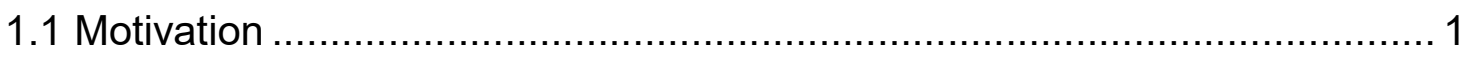

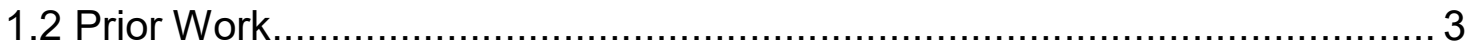

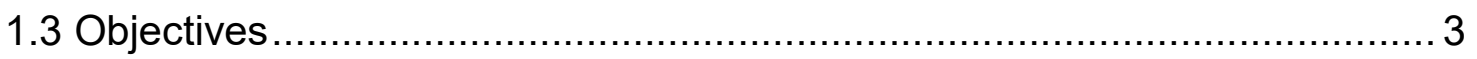

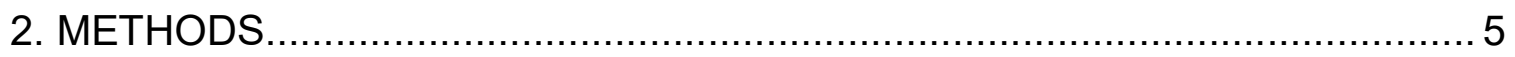

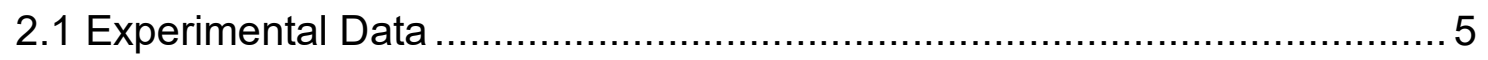

2.1.1 Subject Information ................................................................ 5

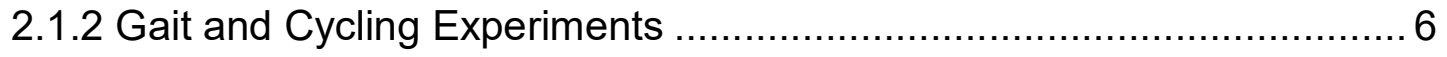

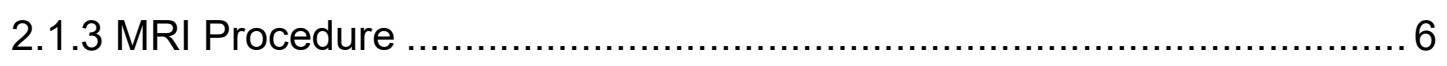

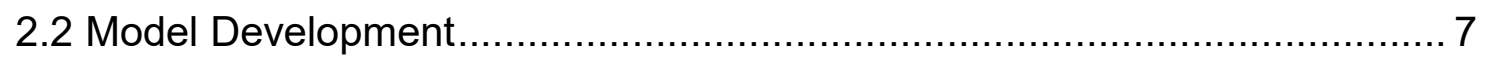

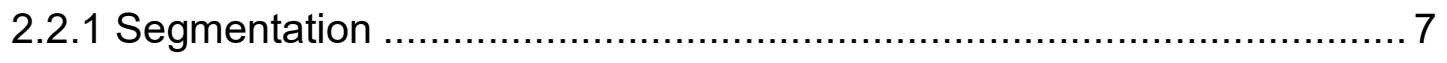

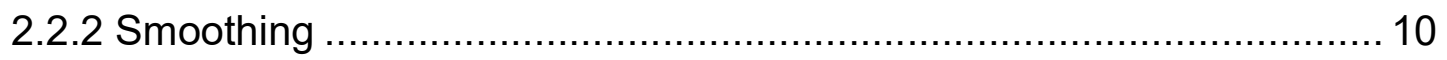

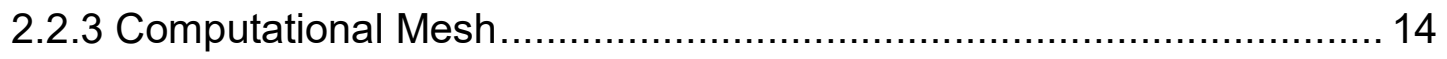

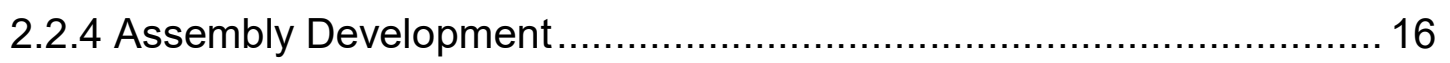

2.2.5 Ligament and Meniscal Horn Definition ........................................ 17

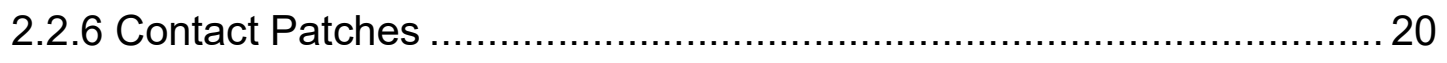

2.2.7 Knee Joint Center and Flexion-Extension Axis ............................... 22 


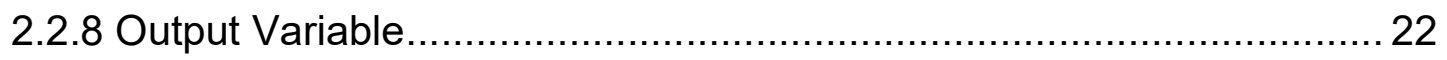

2.2.9 Solution Steps and Boundary Conditions ...................................... 24

2.3 Model Convergence and Validation .................................................. 26

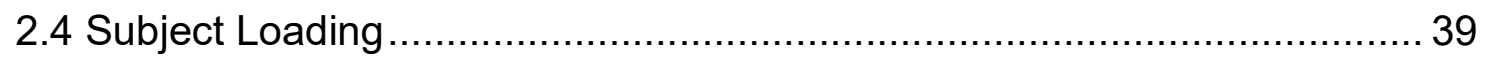

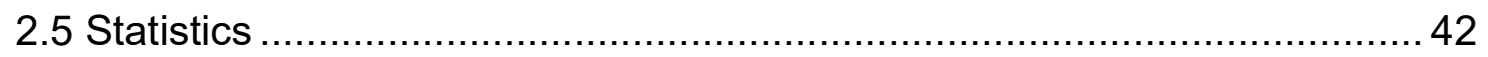

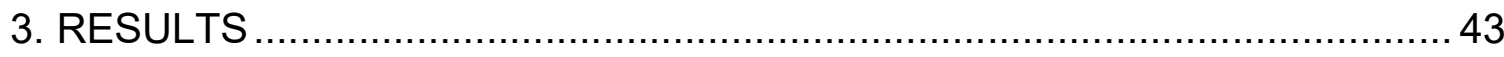

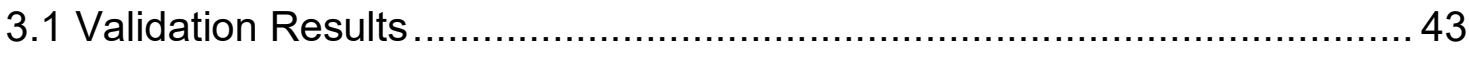

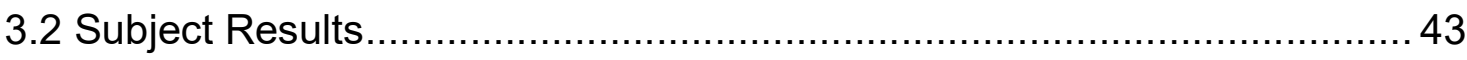

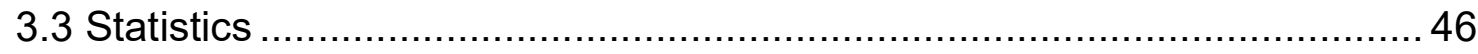

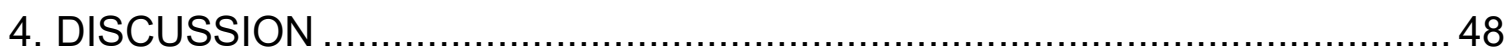

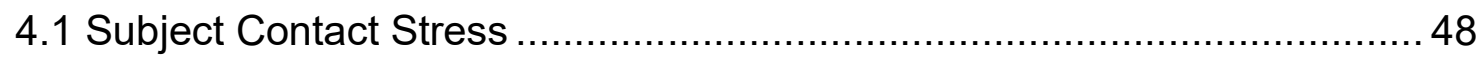

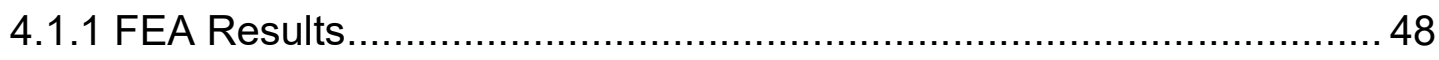

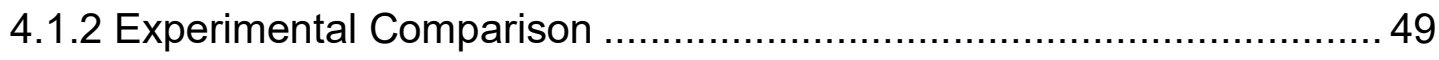

4.1.3 Development Procedure ............................................................... 51

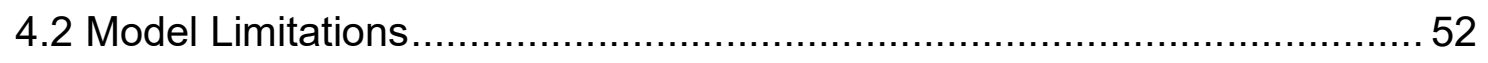

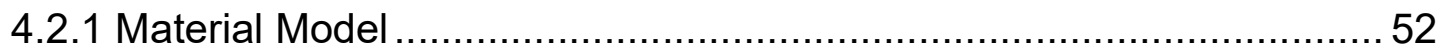

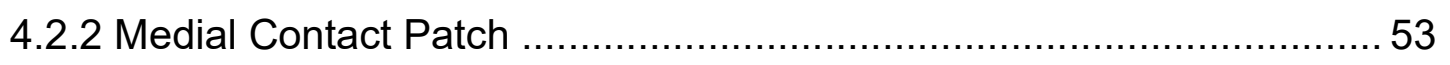

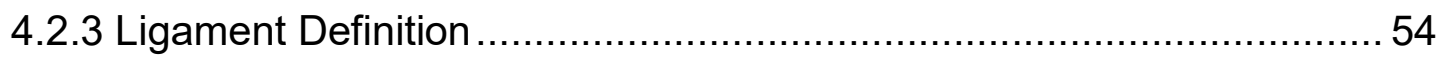

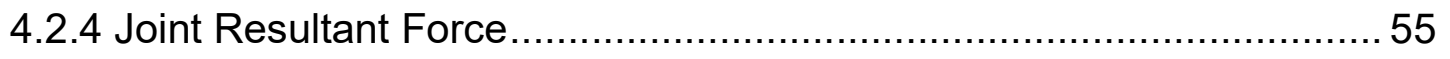

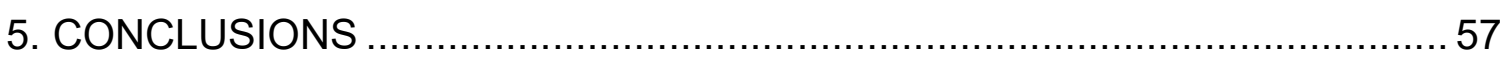

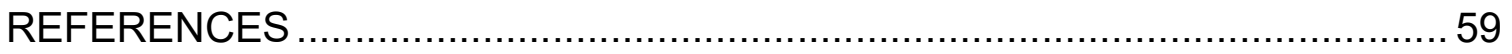

\section{APPENDICES}

A: GAIT AND CYCLING CONTOUR PLOTS ...................................... 70 


\section{LIST OF TABLES}

Table

Page

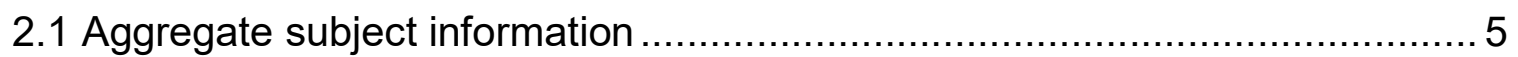

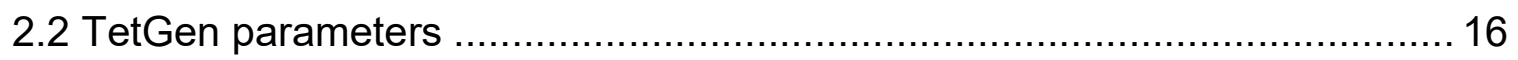

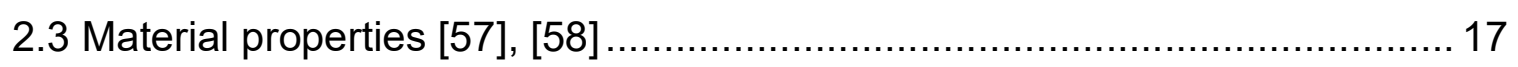

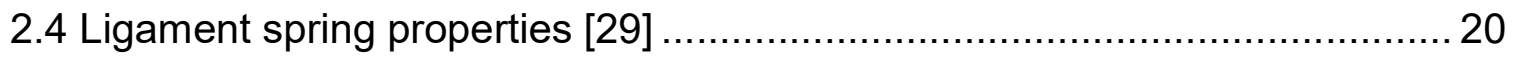

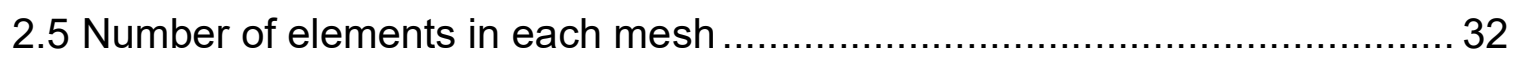

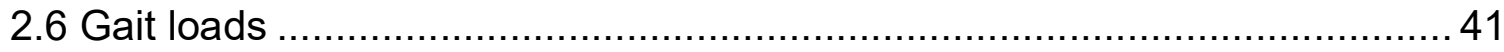

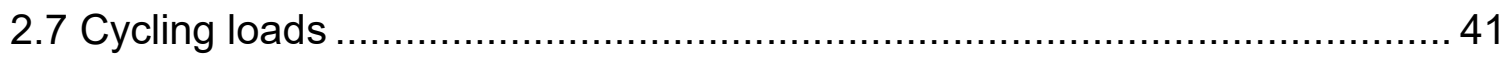

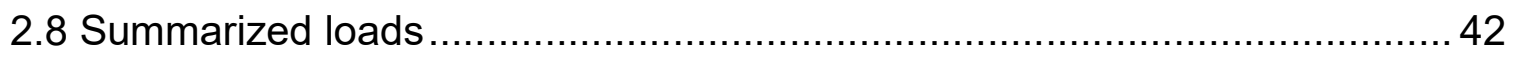

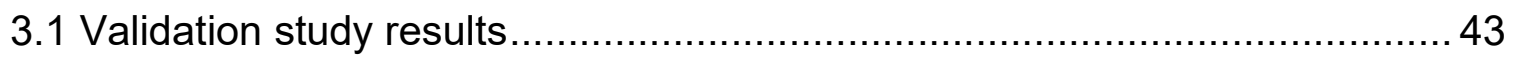

3.2 Actual and normalized gait maximum cartilage contact pressure [MPa] ...... 45

3.3 Actual and normalized gait maximum cartilage contact pressure,

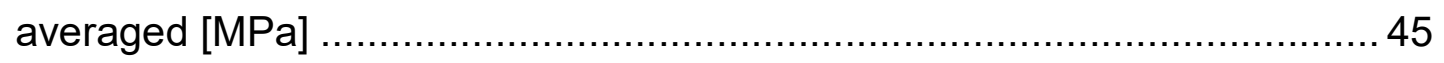

3.4 Actual and normalized cycling maximum cartilage contact pressure [MPa] . 45

3.5 Actual and normalized cycling maximum cartilage contact pressure,

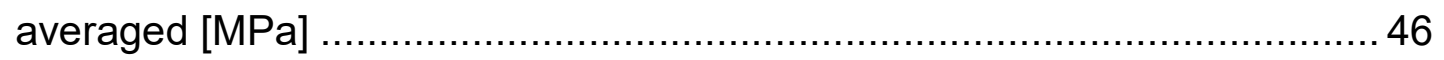

3.6 ANOVA statistical results $\left({ }^{*}\right.$ indicates a significant result, $\left.p<0.05\right) \ldots \ldots \ldots \ldots . \ldots 46$

3.7 Lateral post-hoc Tukey pairwise comparison results ( ${ }^{*}$ indicates a

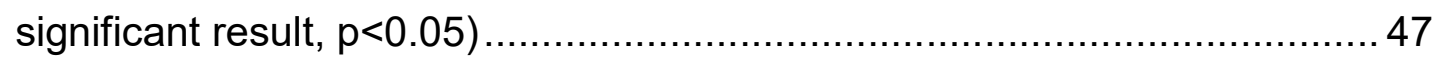




\section{LIST OF FIGURES}

Figure

Page

2.1 Fully segmented knee slice showing femur (red), femoral cartilage (purple) medial meniscus (yellow), medial tibial cartilage (orange), and

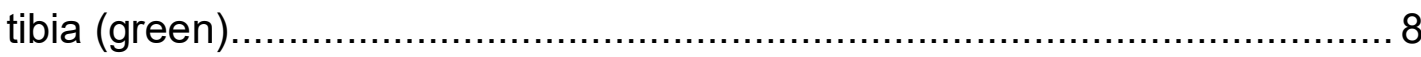

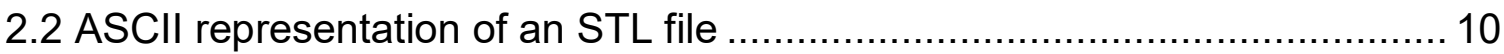

2.3 Before (left) and after (right) smoothing of the medial tibial cartilage.......... 11

2.4 Force displacement curve of the ACL [29] ...................................... 19

2.5 Location of the nodes used for contact pressure averaging $\ldots \ldots \ldots \ldots \ldots \ldots \ldots \ldots \ldots \ldots \ldots \ldots \ldots$

2.6 Boundary conditions and loading in the third analysis step .................... 26

2.7 Convergence study reference nodes for the femoral cartilage (top), medial tibial cartilage (left) and lateral tibial cartilage (right) .................. 28

2.8 Convergence study results for the femoral cartilage (top), medial tibial cartilage (middle), and lateral tibial cartilage (bottom) ........................ 30

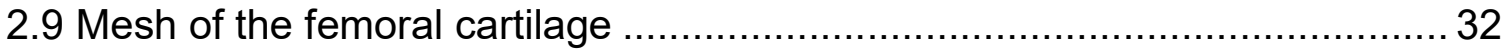

2.10 Mesh of the lateral tibial cartilage ............................................. 33

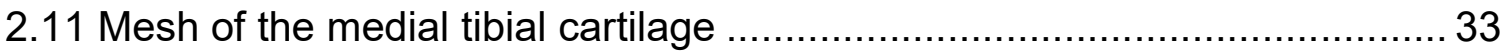

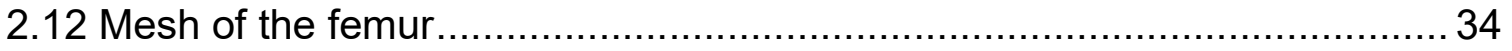

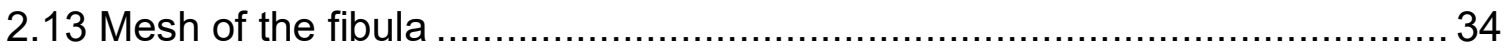

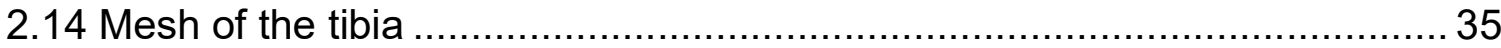

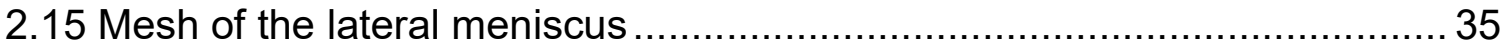

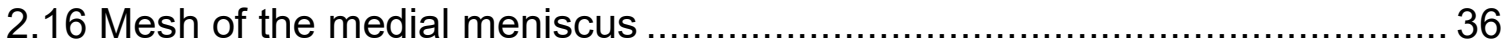


2.17 Whole knee model without femur or femoral cartilage .............................. 36

2.18 Whole knee model viewed from the anterior direction ............................. 37

2.19 Whole knee model viewed from the posterior direction .......................... 38

3.1 Summarized maximum contact pressure results without normalization

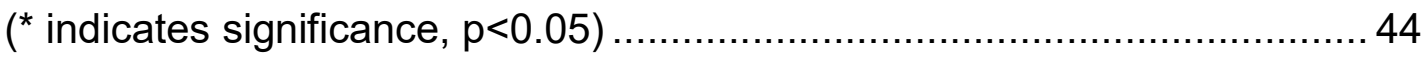

4.1 Typical gait contour plot showing poor medial contact patch......................54

A.1 Control subject contour plots in gait .................................................... 70

A. 2 Control subject contour plots in gait (cont.) ….................................... 71

A.3 Control subject contour plots in cycling ……..................................... 72

A.4 Control subject contour plots in cycling (cont.) ....................................... 73

A.5 Amputee subject contour plots in gait ..................................................... 74

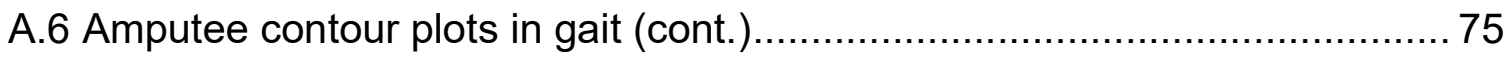

A.7 Amputee subject contour plots in cycling …….................................. 76

A.8 Amputee subject contour plots in cycling (cont.) ….............................. 77 


\section{INTRODUCTION}

\subsection{Motivation}

Osteoarthritis $(\mathrm{OA})$ is a highly debilitating disease that is characterized by the degradation of joint articular cartilage (AC), a near frictionless avascular tissue present in skeletal joints where it covers the articular surfaces of bones [1]. Without a layer of $A C$, joints may become stiff and painful to articulate. It is estimated that the economic burden of OA in America totals more than $\$ 200$ billion annually [2]. This is from both treatment costs and economic costs associated with lost productivity. OA is the most common cause of disability in adults, with approximately 30.8 million American adults suffering from OA in 2015 [3]. For military veterans specifically, roughly 1 in 3 has OA [4]. Given that there were 20.8 million veterans in the US population in 2015 [5], there are roughly 6.2 million veterans currently living with $\mathrm{OA}$, and it is one of the most common causes of pain and disability in veterans. In 2017, the United States Department of Veterans Affairs (VA) spent $\$ 68.6$ billion on healthcare [6]. Because American taxpayer funds go towards the treatment of OA for veterans, there is a large economic incentive to understand more about the disease and how it can be prevented.

From cohort studies done on veterans [4], [7], it is understood that transtibial (TT) amputees (leg amputated below the knee) have a much higher incidence of tibiofemoral (TF) OA than non-amputees, particularly in their healthy knee. The leading belief is that significant trauma to a lower limb causes the body to naturally compensate by loading the intact native limb more heavily [8]-[11]. 
This can lead to loading the AC in modes it was not designed for and at higher loads. This abnormal loading condition causes higher contact stresses inbetween bodies of the knee, which can lead to higher AC strains and therefore accelerated wear [12], [13]. Therefore, the most direct measure of OA risk due to AC loading is the contact pressure.

Further, it is not well understood how altering exercise routines for TT amputees impacts the useful life of the TF cartilage. Experimental evidence points to the trend that non-impact exercises, such as cycling, have lower peak cartilage loads than impact-intensive exercises such as normal gait or running [14], [15]. However, the loads present at the TF joint are multi-dimensional due to the complex muscle structure in the thigh and shank that transmit forces to and across the joint. In general, there are 3 forces [compressive, anterior-posterior (AP) shear, and medial-lateral (ML) shear) and 3 rotation moments (flexionextension (FE), varus-valgus (VV), and internal-external (IE)] being applied. Additionally, the contact geometry of the TF joint is a function of the flexion angle. Therefore, while the compressive force may be different in one exercise compared to another, the addition of shear forces and rotation moments compounded by the changing joint angle means that understanding the relationship between exercise type and contact pressure is not trivial. Determination of this relationship is best suited to a numerical simulation. 


\subsection{Prior Work}

Finite element models have been widely used to investigate the effects of various physiological conditions on TF biomechanics. A large body of literature is available for the modeling of different materials in the TF joint, such as cartilage [16]-[21], menisci [22]-[25], bones [26]-[28], and ligaments [29]-[32]. Other authors have proposed generalized development methods for creation of joint models [33], [34].

Whole knee FEA models have been used to simulate patellofemoral mechanics [35], [36] and how they can impact patellofemoral cartilage wear [37]. Studies have also investigated how knee trauma and surgery impacts stress distribution and biomechanics [38]-[41], carrying some clinical implication on how to better treat patients with such conditions. Probabilistic models have highlighted knee model sensitivity to soft tissue definitions and why effective subject-specific modeling is paramount to producing clinically relevant data [42]-[44]. Previous studies within the Cal Poly Human Motion Biomechanics Lab have created TF models to investigate effects of exercise choice [45], obesity and joint malalignment [46], and anterior cruciate ligament (ACL) deficiency [47] on cartilage stresses. In the literature search conducted, a study investigating exercise choices for TT amputees was not found.

\subsection{Objectives}

The objective of this study is to investigate cartilage pressure differences between exercise types as well as between TT amputees and healthy control 
subjects. Gait and cycling are two of the most common and accessible exercise types. They also constitute an important subset as they represent two different loading modalities (impact and weight bearing in gait versus non-impact and nonweight bearing in cycling). By analyzing differences between TT amputees and controls, relative risks specific to TT amputees can be highlighted.

These risk factors will be assessed by creating TF FEA models to predict the cartilage contact pressure in TT amputees and control subjects. By use of magnetic resonance imaging (MRI), the joint geometry can be recreated as a computer model. Subsequently applying material properties, boundary conditions, contact definitions, and ligament springs will yield a model that can recreate in vivo TF contact pressure. The model will be validated against experimental measures of knee contact stress and tested for sufficient numerical convergence. Once converged and validated, the model can be used to simulate loading cases from several TT amputee and control subjects for gait and cycling exercises. From these results, any statistical differences can be found.

Another objective of the study is to develop a method by which multiple models can be formed. The single model used for results will serve as a proof-ofconcept for the process that can then be used to create subject-specific models in the future. The addition of subject-specific geometry will increase the clinical relevance of the study results as a subject could then have their specific knee loads applied directly to a model of their knee for analysis of how exercise changes would impact their quality of life, instead of relying on the assumptions of a generalized model. 


\section{METHODS}

\subsection{Experimental Data}

As part of the larger scope of this research project, 8 TT amputees and 11 healthy control subjects were brought in for motion analysis. Of these, 6 amputees and 6 control subjects had their data used for this FEA study.

\subsubsection{Subject Information}

Aggregate subject demographics are presented in Table 2.1. All subjects were admissible within the exclusion criteria for the motion analysis study. Control subjects were selected to match the demographics of the amputee group. There were a limited number of readily available amputee subjects, and only six were available for the FEA study.

Table 2.1 Aggregate subject information

\begin{tabular}{|c|c|c|c|c|c|c|}
\hline Status & Subject ID & $\begin{array}{l}\text { Measured } \\
\text { Leg }\end{array}$ & Gender & $\begin{array}{r}\text { Height } \\
{[\mathrm{m}]}\end{array}$ & $\begin{array}{r}\text { Mass } \\
{[\mathrm{kg}]}\end{array}$ & $\begin{array}{r}\text { BMl } \\
{\left[\mathrm{kg} / \mathrm{m}^{\wedge} 2\right]}\end{array}$ \\
\hline \multirow[t]{6}{*}{ Control } & 2016Aug12-02 & Right & Male & 1.82 & 82.6 & 25.0 \\
\hline & 2016Aug19-01 & Left & Male & 1.75 & 88.5 & 28.9 \\
\hline & 2016Nov05-01 & Right & Male & 1.79 & 61.2 & 19.1 \\
\hline & 2016Aug15-01 & Right & Male & 1.79 & 86.6 & 27.0 \\
\hline & 2016Aug26-01 & Right & Female & 1.71 & 76.7 & 26.2 \\
\hline & 2016Sep01-01 & Left & Male & 1.85 & 82.6 & 24.1 \\
\hline \multirow[t]{6}{*}{ Amputee } & 2016 Nov10-01 & Left & Male & 1.82 & 74.8 & 22.6 \\
\hline & 2016Nov14-02 & Right & Male & 1.69 & 83.9 & 29.4 \\
\hline & 2017Mar18-01 & Right & Male & 1.81 & 80.7 & 24.6 \\
\hline & 2017Nov11-01 & Left & Female & 1.77 & 92.2 & 29.6 \\
\hline & $20170 c t 30-01$ & Right & Male & 1.80 & 72.4 & 22.3 \\
\hline & 2017Sep09-01 & Right & Male & 1.78 & 80.6 & 25.4 \\
\hline
\end{tabular}




\subsubsection{Gait and Cycling Experiments}

As part of a separate study, other researchers in the group conducted experiments according to [14] and used inverse dynamics to calculate knee joint resultant loads. All study protocols were approved by Cal Poly's Human Subjects Committee and were designed to minimize risk to human subjects. From the experiments, knee resultant loads were determined including the knee compressive load, anterior-posterior and medial-lateral shear forces, varusvalgus and internal-external rotation moments, and flexion angles.

\subsubsection{MRI Procedure}

One control subject was asked to undergo a MRI scan at San Luis Obispo's French Hospital as part of the testing procedure. The MRI was performed on a GE Signa HDxt 1.5T scanner (GE Healthcare, Little Chalfont, UK). The MRI was a proton density fast spin-echo, fat saturated sequence (4800 second relaxation time, 32.1 second echo time, 2 averages, 90-degree flip angle) in the sagittal plane with $1 \mathrm{~mm}$ slice thickness and a $512 \times 512$ matrix. The MRI covered approximated $8 \mathrm{~cm}$ of the distal femur and $8 \mathrm{~cm}$ of the proximal tibia. After investigating other sequences and on the advice of a local radiologist, this sequence was chosen to give the highest signal and make the cartilage easier to segment. The MRI was anonymized using a MATLAB (MathWorks, Natick, Massachusetts, USA) script to remove personal subject details then slightly filtered and color balanced to assist with segmentation. 


\subsection{Model Development}

Once the MRI was obtained and anonymized, it had to be turned into an accurate 3D model of the TF geometry, including any relevant soft tissue. From there, the body had to be turned into a computational mesh suitable for FEA. Because of the complicated geometry and wanting to establish a procedure to develop multiple models, an automated process for developing the computational mesh was desired. By using tetrahedral elements of sufficient complexity, a Delaunay tetrahedralization scheme produced suitable meshes.

\subsubsection{Segmentation}

The separate bodies within the TF joint were outlined and shaded, a process referred to as segmentation, using an open source program, ITK-SNAP [48] (University of Pennsylvania, Philadelphia, PA, USA). The segmentation

process involved assigning different color labels to specified areas of the MRI. To obtain sufficient geometry of the TF joint, the distal femur, femoral cartilage, lateral and medial menisci, lateral and medial tibial cartilage, proximal femur, and fibula were all segmented. The knee ligaments did not appear in the MRI in enough detail to be faithfully segmented for construction of a continuum body. Therefore, they were excluded from segmentation and modeled as non-linear springs (section 2.2.5) with origin and insertion sites determined from the MRI. An example of the completed segmentation is shown in Figure 2.1. 


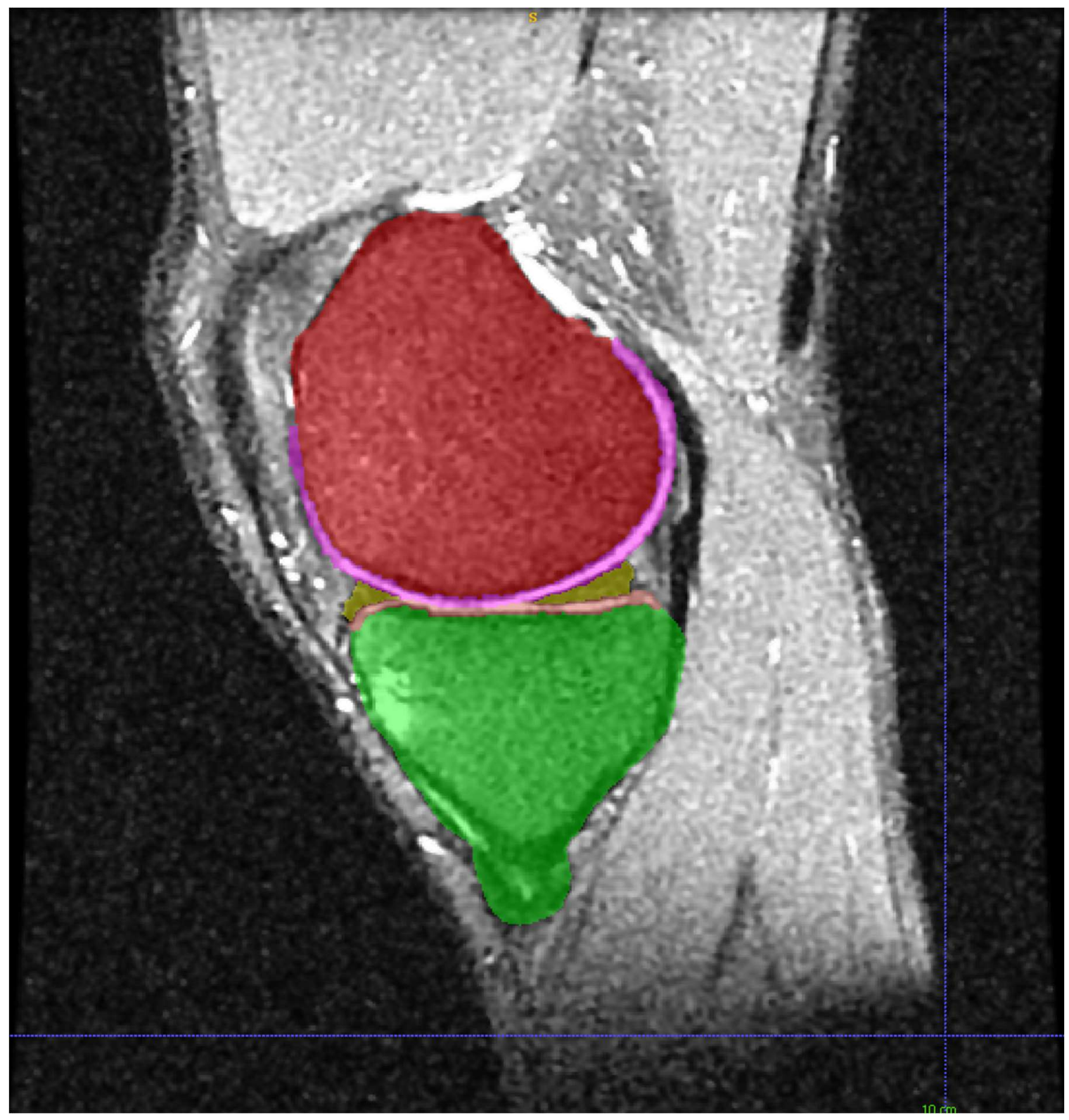

Figure 2.1 Fully segmented knee slice showing femur (red), femoral cartilage (purple) medial meniscus (yellow), medial tibial cartilage (orange), and tibia (green).

After segmentation was complete, ITK-SNAP assembled the labels from each slice into a 3D body by taking the outline of the segmented area in the slice and stitching them together to create a surface. One of the most direct ways to represent the surface mesh is with a 3D Piecewise Linear Complex (PLC) [49]. A 
PLC is a set of cells with the properties that: 1) the boundary of each cell in the PLC is a union of cells in the PLC, and 2) if two distinct cells intersect, their intersection is a union of cells in the PLC. A 0-dimensional cell of the PLC is a "node" or "vertex", a 1-dimensional cell is an "edge" or "segment", and a 2dimensional cell is a "face" or "facet". From the cloud of points representing the surface of the body, a PLC can be defined with triangular facets that closely approximates the true underlying surface.

Computationally, triangular 3D PLCs are often represented as stereolithography files (STL). When shown in plain text, STL files list the facets of the PLC with the 3D coordinates of each facet's nodes. STL files are commonly used in computer applications, notable in 3D printing applications. Exporting the knee structures in this format made them readily available to be imported into a wide variety of solid modeling software. STL files can be represented in either binary or ASCII characters. ASCII characters resulted in a larger file, but were easier to import into MATLAB. This file format is shown in Figure 2.2 


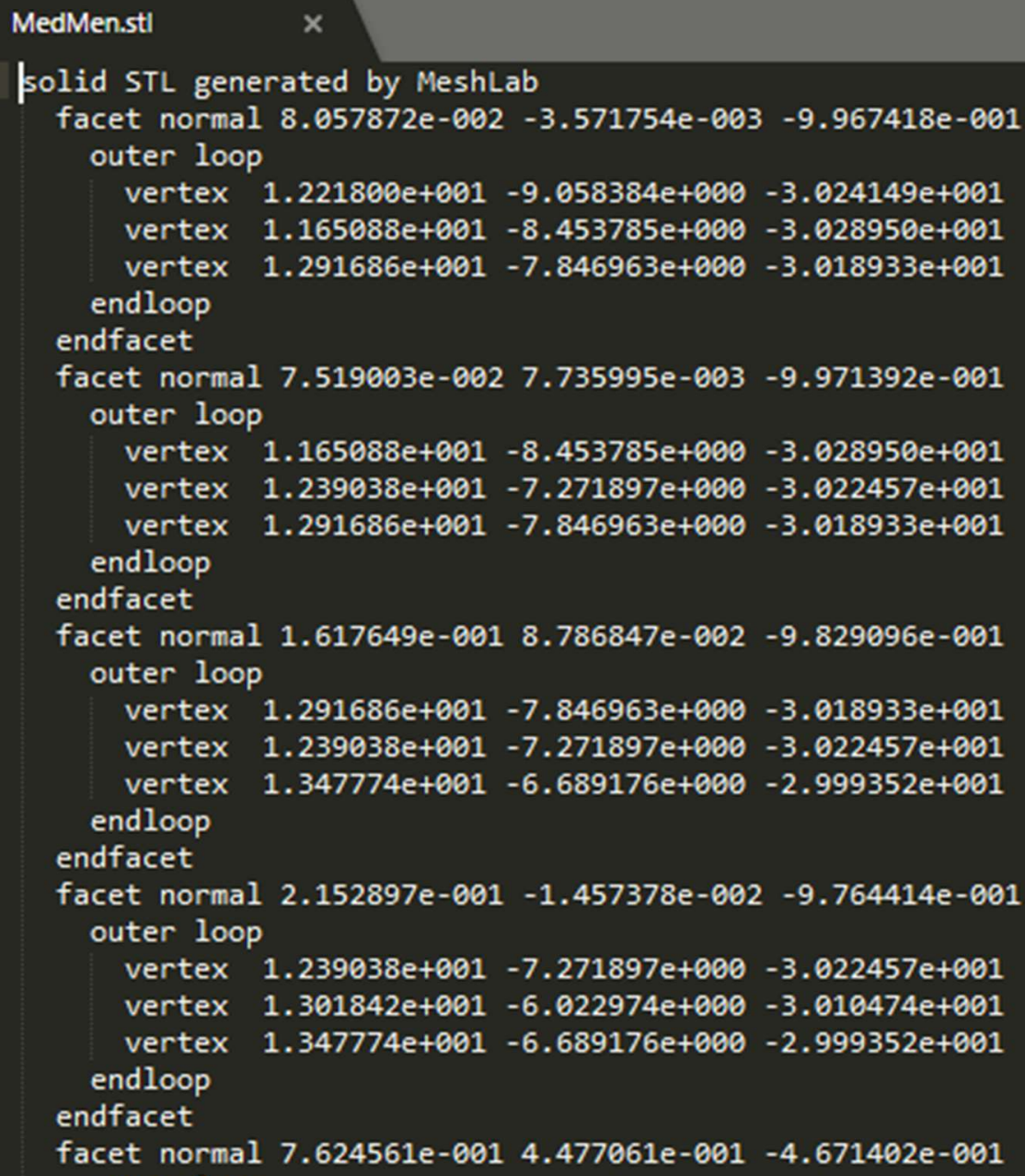

Figure 2.2 ASCll representation of an STL file.

\subsubsection{Smoothing}

The MRI only afforded limited resolution of the knee structures. Because there were only approximately 100 sagittal slices, the bodies exported from ITKSNAP were very coarse and had sharp steps. Additionally, because each segmented voxel was treated as a data point, the raw mesh density was much higher than necessary, often by an order of magnitude or more. To reflect the smooth anatomical surface more accurately, the bodies were refined in the opensource mesh processing tool MeshLab [50] (Institute for Computer Science and 
Technologies, Pisa, Italy). Within MeshLab, a series of processing filters were applied to turn the raw surfaces into ones suitable for computational work. Before and after representations of the smoothing process are shown in Figure 2.3.

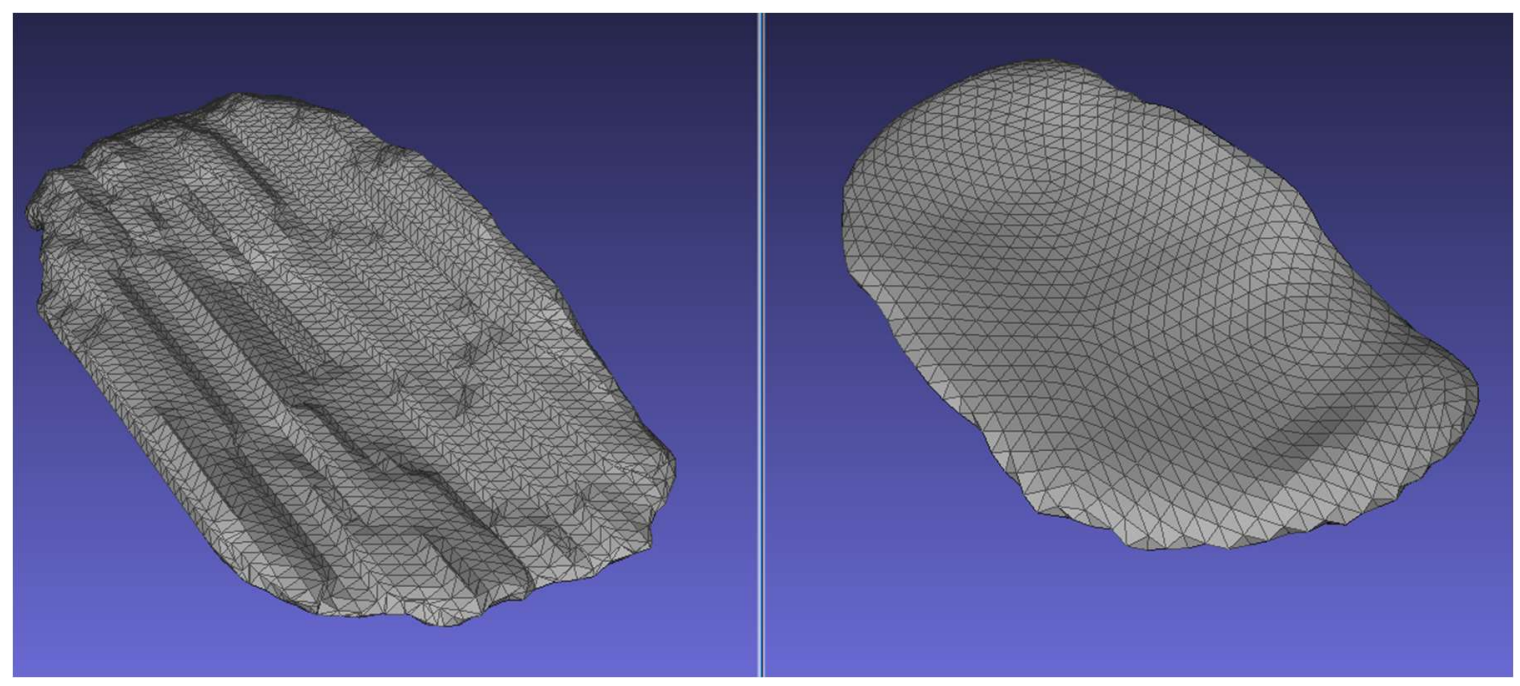

Figure 2.3 Before (left) and after (right) smoothing of the medial tibial cartilage.

The first filter applied was a Taubin two-step smooth. The Taubin method is a series of two Laplacian smoothing steps [51], [52]. Gaussian smoothing adjusts each node of the surface by a weighted average of its $1^{\text {st }}$-order neighbors using a scaling factor, $\lambda$, between 0 and 1 . This works for local smoothing, however to get global smoothing over the entire body, Gaussian smoothing needs to be applied iteratively many times. Because of the weighted average method of smoothing, this makes all nodes tend to move towards each other, leading to global shrinkage of the body. Additionally, any spikes in the raw mesh can become exacerbated with large numbers of iterations. The Taubin approach is to use two successive Gaussian steps, with the second one having a negative scale factor, $\mu$, larger in magnitude than $\lambda$. That is, $0<\lambda<-\mu$. The effect of this second step with a negative scale factor is to create a low pass filter. By varying 
the number of iterations and the $\lambda$ and $\mu$ parameters, the transfer function of the filter can be tuned. The $\lambda$ and $\mu$ parameters and the number of iterations were selected to give a qualitatively smooth surface while quantitatively minimizing volume shrinkage. Setting $\lambda=0.8$ and $\mu=-0.83$ with 1000 iterations, net volume change from the raw to smooth STL files was measured to be between 0 and $3 \%$. A slight volume decrease was desirable as it prevented the structures from having a high degree of contact over-penetration in the reference configuration.

After the body was smoothed, the number of faces needed to be reduced. Because of the high mesh density output by ITK-SNAP, it would be computationally excessive to turn the smooth surfaces directly into computational meshes. Additionally, in anticipation of the final mesh processing step, isoparameterization (IP), the number of faces needed to be reduced to minimize computational time. MeshLab's Quadric Edge Decimation (QED) feature was used to cut the number of faces down by a factor of 2 to 4 , depending on the geometry. QED collapses surface nodes together to get to the user-specified target number of faces. At the same time, the filter takes care to preserve the topology and boundary of the mesh. A quality threshold between 0 and 1 was used to specify how much MeshLab penalized poor quality faces. This parameter was set to 1 for all meshing.

After QED, the mesh was sufficiently smooth, but the size and arrangement of the surface faces were highly irregular. For defining the contact geometry, this irregular facet distribution was difficult to work with. To create a more regular surface, the body was re-meshed using an IP filter [53]. This filter 
mapped the surface mesh into an abstract mesh domain. It did this by breaking sections of the mesh up into a larger triangular matrix. Upper and lower bounds for the number of triangles in this abstract domain were provided, generally around 15 and 50, respectively. The algorithm optimized the abstract mesh to produce regular mesh boundaries. Then, within each larger triangle, the faces were restructured to yield a uniform mesh distribution while preserving the body topology. Once these larger triangles restructured the faces within their boundaries, the entire meshed was transformed back into the present domain. A second re-meshing parameter defined the mesh density within each of the abstract mesh faces. This allowed control of the final mesh density and was used for the convergence study.

The IP filter was not perfectly stable. Sometimes, unstable points would appear in the abstract mesh domain and become exacerbated in the real domain, leading to areas of high aspect ratio, irregular elements. It is not known what exactly caused these points, however they most frequently occurred on bodies that were very thin with high levels of curvature, e.g., the femoral cartilage. This problem was rectified by increasing both the min and max abstract mesh size. By allowing the abstract mesh to have more degrees of freedom, it was less likely that instability points would occur. Because of this phenomenon, the ranges of abstract mesh sizes were not consistent between bodies or subjects. However, overall, they ranged from 15 to 100 faces.

As mentioned previously, once the abstract mesh was created, the second step was to apply a re-meshing parameter that subdivided the abstract mesh into 
uniform meshes in the real domain. Again, this parameter varied between bodies and subjects, and was chosen to create a mesh with a target number of faces for the structure in question. Over all the meshes, this parameter ranged from 4 to 12. After all meshing filters were applied and the mesh was observed to be sufficiently smooth, uniform, and with the target number of faces, it was exported from MeshLab as an ASCII text STL file.

\subsubsection{Computational Mesh}

For generation of the computational mesh, the Delaunay tetrahedralization scheme TetGen (Weierstrass Institute for Applied Analysis and Stochastics, Berlin, Germany) was used [49]. TetGen uses constrained Delaunay tetrahedralizations to construct a tetrahedral mesh from a surface mesh. The output of the TetGen algorithm was the original surface topology with tetrahedral elements through the volume of the body.

For finite element simulations, results are calculated at integration points via approximation functions and interpolated to the nodes. For triangular (tetrahedral in 3D) elements, linear approximation functions often have higher degrees of error and result in a stiffness matrix that is artificially stiffer than it truly is [54]. Therefore, linear tetrahedral elements are often avoided. Hexahedral elements give more accurate approximations for similar number of degrees of freedom (DOFs). However, 10 node tetrahedral elements (4 vertices and midpoints of the 6 edges) or 15 node tetrahedral elements (centroids of 4 faces and centroid of element) can give comparable results to 8 node hexahedral 
elements with lower computational time [54]. TetGen can output either 4 node or 10 node elements, therefore it was configured to output 10 node elements. If 15 node capability is added to TetGen in the future, that refinement would be a good option for model optimization.

Because of the triangular facets used to define a given body, the mesh can be described as a simplicial complex. That is, a group of 2-simplices representing a set of points. A d-simplex is the d-dimensional notion of a triangle. E.g. 0-simplex is a single point, a 1-simplex is a line segment, a 2-simplex is a triangle, etc. Now, let $\mathrm{V}$ be a set of points in $\mathrm{R}^{\mathrm{d}}$. A triangulation of $\mathrm{V}$ is a simplicial complex whose vertex set is a subset of $\mathrm{V}$. A Delaunay triangulation is a robust method to triangulate a set of points while preserving complex geometry. If $\sigma$ is a k-simplex $(0 \leq k \leq d)$ in $V$, a circumsphere of $\sigma$ is a sphere that passes through all the vertices of $\sigma . \sigma$ is said to be Delaunay if there exists a circumsphere such that no vertex of $\mathrm{V}$ lies inside of it. A Delaunay triangulation of $\mathrm{V}$ is a simplicial complex such that all simplices are Delaunay. In $\mathrm{R}^{3}$, this is called a Delaunay tetrahedralization. For generation of the computational mesh, a Delaunay tetrahedralization scheme was used.

TetGen was implemented as part of the Geometry and Image-Based Bioengineering add-On (GIBBON) [55] for MATLAB. A MATLAB script imported all the STL files for a given subject, designated which bodies were soft tissue (i.e. needed to have a tetrahedral computational mesh), ran those bodies through TetGen, then wrote the entire assembly into an input file (INP) to be used in Abaqus (Dassault Systems, Providence, Rhode Island, USA). TetGen used 
specific parameters written in a command line switch string to control the quality of the output mesh. All bodies were meshed with the string "-pq5/15Aa10VO7". Each command and its impact are summarized in Table 2.2. Further explanation of the switch command and each available option can be found in the TetGen manual [49].

Table 2.2 TetGen parameters

\begin{tabular}{|c|c|c|}
\hline $\begin{array}{l}\text { Switch } \\
\text { command }\end{array}$ & Value & Description \\
\hline$-p$ & N/A & Input is a PLC. \\
\hline$-q$ & $5 / 15$ & $\begin{array}{l}\text { Set maximum radius-edge ratio to } 5 \text { and } \\
\text { set minimum dihedral angle to } 15 \text { degrees. }\end{array}$ \\
\hline$-A$ & $\mathrm{~N} / \mathrm{A}$ & Assigns region indices. \\
\hline$-\mathrm{a}$ & 10 & Maximum tetrahedron volume. \\
\hline$-V$ & N/A & Detailed terminal output. \\
\hline-0 & 7 & Set mesh optimization level to 7 ( 0 to 10$)$. \\
\hline
\end{tabular}

\subsubsection{Assembly Development}

Once MATLAB output the INP file, it was imported into the Abaqus graphic user interface (GUI). From here, the material properties, interactions, and boundary conditions were defined. Because the bones are several orders of magnitude stiffer than the soft tissue, they were modeled as rigid bodies [26], [56], [57]. This reduced the complexity of the model, cutting down on computational time. Rigid bodies are defined in Abaqus by specifying the elements in the body and a reference point. The reference point acts as the node which all body translations and rotations are defined about. It is also where boundary conditions are applied to. For the femur, the reference point was picked as the knee joint center (KJC). The tibia and fibula were modeled as a single rigid 
body to reduce model complexity. The tibia/fibula reference point was a node selected on the tibial eminence in the middle of the tibial plateau.

Cartilage is most closely modeled as a depth-dependent, biphasic, viscoelastic material due to its anatomical structure [19]. Similarly, the menisci are fiber-reinforced in the annular direction and viscoelastic [22]. However, because the time constant of these materials is high and the loading is applied in a quasi-static manner, both can be accurately represented with a linear elastic model, where material response can be defined by an elastic modulus, E, and a Poisson's ratio, v [57], [58]. Cartilage and menisci material properties are summarized in Table 2.3.

Table 2.3 Material properties [57], [58]

\begin{tabular}{ll|r} 
Tissue & Property & Value \\
\hline Cartilage & $\mathrm{E}[\mathrm{MPa}]$ & 15 \\
& $\mathrm{v}$ & 0.475 \\
\hline Meniscus & $\mathrm{E}[\mathrm{MPa}]$ & 59 \\
& $\mathrm{v}$ & 0.48
\end{tabular}

\subsubsection{Ligament and Meniscal Horn Definition}

In previous studies, ligaments were modeled as 3 dimensional bodies with hexahedral elements [46], [47]. This caused issues when the ligaments were put into compression. Anatomically, ligaments can support minimal compressive loads. Trying the replicate this attribute in the Abaqus model caused buckling stability issues in compression. It was solved by including an artificially viscous dampening force, however that also impacted the final contact stress result. In other finite element studies of the knee, ligaments have been modeled as 
collections of spring elements to prevent this [57], [59], [60]. The medial collateral ligament $(\mathrm{MCL})$ and lateral collateral ligament (LCL) were modeled using three spring elements (anterior, superior, and posterior bundles) while the $A C L$ and posterior cruciate ligament (PCL) were modeled using two spring elements (anteromedial $(\mathrm{AM})$ and posterolateral $(\mathrm{PL})$ bundles for the $\mathrm{ACL}$ and anterior and posterior bundles for the $\mathrm{PCL}$ ). Collateral ligament bundles are named for their location relative to the other bundles. For example, the superior bundle of the LCL inserts slightly superiorly to the insertion sites of the anterior and posterior bundles. Cruciate ligaments are named for where they insert into the tibia. For example, the AM bundle of the ACL inserts more anteriorly and medially than the PL bundle, while the ligament itself inserts on the anterior aspect of the tibia. Insertion sites were determined by cross-referencing the model with the MRI. An accurate physiological model represents these springs with non-linear force displacement curves [29]. These spring curves include zero force in compression and a linear region in tension, connected by a non-linear toe region. Also, the graphs were offset to give a level of prestress in the reference configuration.

The reference length, $I_{r}$, of each ligament was the distance between the origin and insertion sites. This reference length was multiplied by a scaling factor, $\beta$, to calculate the zero-load length, lo. Displacements, $u$, from the reference length were mapped into strain values relative to the zero-load length by

$$
\varepsilon=\frac{u+l_{r}-l_{o}}{l_{o}}
$$

Using a spring stiffness parameter, $k$, and a reference strain, $\varepsilon_{r}=0.03$, force was defined as a piecewise function of strain according to [29] 


$$
f(x)=\left\{\begin{aligned}
\frac{1}{4} k \varepsilon^{2} / \varepsilon_{r}, & 0 \leq \varepsilon \leq 2 \varepsilon_{r} \\
k\left(\varepsilon-\varepsilon_{r}\right), & \varepsilon>2 \varepsilon_{r} \\
0, & \varepsilon<0
\end{aligned}\right.
$$

The spring definition was input into Abaqus with force as a function of displacement. The force displacement curve for the $\mathrm{ACL}$ is shown in Figure 2.4

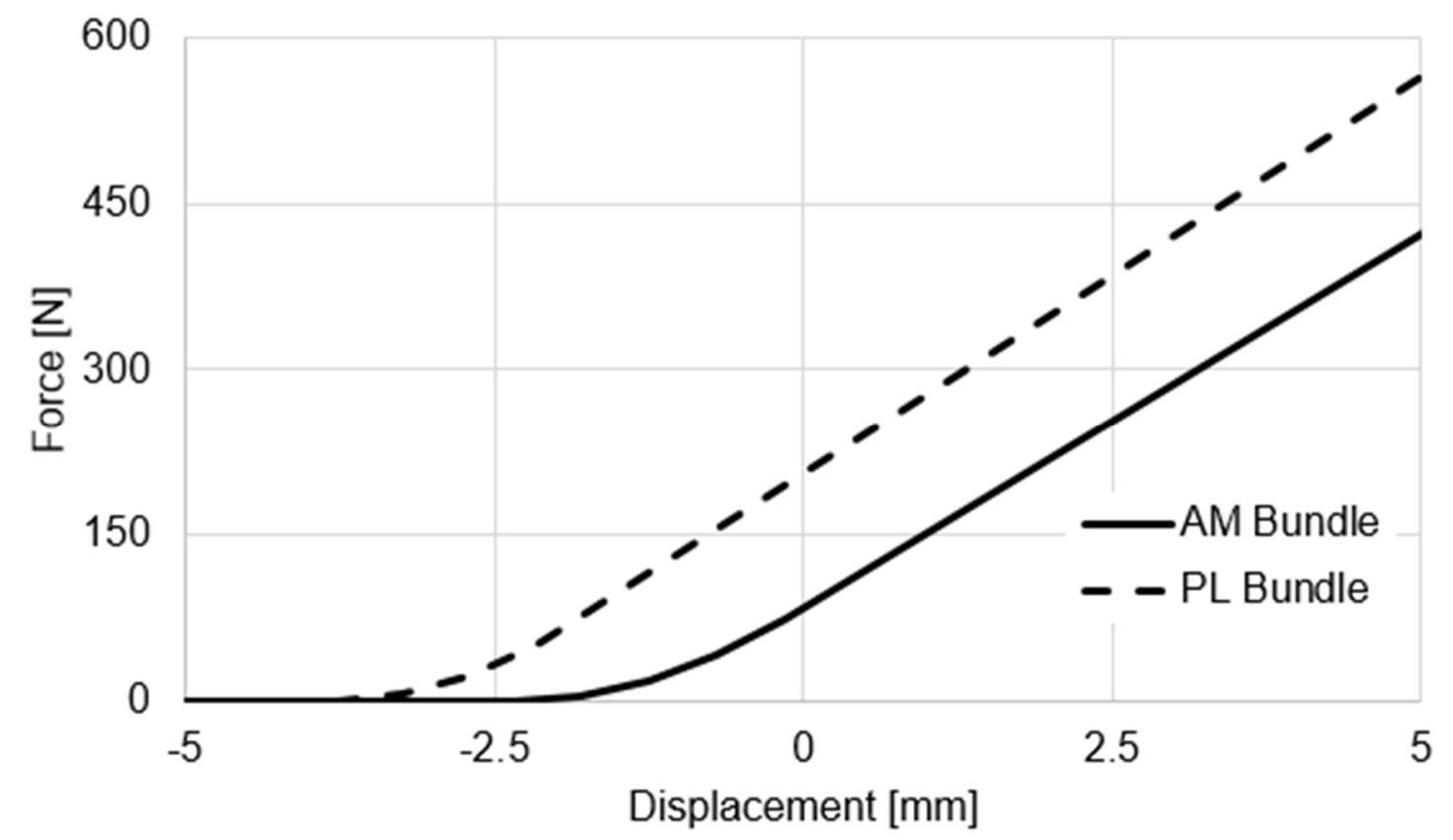

Figure 2.4 Force displacement curve of the ACL [29].

All the ligament spring properties are given in Table 2.4. Insertion and attachment sites were manually selected on the model by referencing the MRI data. The spring definitions were calculated in MATLAB and written into the proper Abaqus syntax. The MATLAB script then wrote the spring elements into the INP file. 
Table 2.4 Ligament spring properties [29]

\begin{tabular}{ll|rr} 
Ligament & Bundle & $\mathrm{k}$ & $\beta$ \\
\hline $\mathrm{ACL}$ & $\mathrm{AM}$ & 2500 & 0.94 \\
& $\mathrm{PL}$ & 2500 & 0.90 \\
\hline $\mathrm{LCL}$ & Anterior & 2000 & 1.25 \\
& Posterior & 2000 & 0.92 \\
& Superior & 2000 & 1.05 \\
\hline $\mathrm{MCL}$ & Anterior & 2750 & 0.96 \\
& Posterior & 2750 & 0.97 \\
& Inferior & 2750 & 0.96 \\
\hline $\mathrm{PCL}$ & Anterior & 5100 & 1.24 \\
& Posterior & 3000 & 1.03
\end{tabular}

The menisci were constrained by means of the meniscal horns, which were modeled as bundles of linear springs. Each horn had 16 spring elements that were attached between various points on the horn face and a single point on the tibial plateau. Selecting 16 different points spread the force of the horn out over the surface of the face, alleviating stress concentrations. The tibial attachment point for each horn was picked so that the springs would be tangential to the annular direction of the meniscus. Horn spring properties were selected similar to Haut-Donahue, et. al. [57]. These springs were written in MATLAB and included in the Abaqus INP file in the same fashion as the ligament springs.

\subsubsection{Contact Patches}

Most of the complexity in making a FEA model of the knee came from the contact between the articular surfaces. There were six contact patches in the TF joint that were included in the FEA models. They were:

- Lateral femoral cartilage/lateral meniscus 
- Lateral femoral cartilage/lateral tibial cartilage

- Lateral tibial cartilage/lateral meniscus

- Medial femoral cartilage/medial meniscus

- Medial femoral cartilage/medial tibial cartilage

- Medial tibial cartilage/medial meniscus

To reflect the fact that cartilage is a nearly frictionless material, contact was modeled as frictionless tangent to the surface and "hard" contact normal to the surface [57], [58]. To account for how the contact patches moved during flexion, one large contact patch was defined for each side of the femoral cartilage. Each superior surface of the tibial cartilage was also defined as one contact patch, as opposed to defining a contact patch for the femoral cartilage contact and a second patch for the meniscal contact.

Additionally, there were three bone/cartilage interfaces that needed to be modeled. These were:

- Femur/femoral cartilage

- Tibia/medial tibial cartilage

- Tibia/lateral tibial cartilage

These were imposed by enforcing a tie constraint between the inner surface of the cartilage and the area of the respective bone that it overlapped. Contact patches were manually selected using the Abaqus GUI. 


\subsubsection{Knee Joint Center and Flexion-Extension Axis}

To properly apply the subject loading case, both the KJC and flexionextension (FE) axis had to be defined. The $\mathrm{KJC}$ is the midpoint of the femoral epicondyles and is where knee resultant loads are calculated. The FE axis is the axis defined by the femoral epicondyles and is the axis the femur rotates about.

The FE axis was calculated by selecting nodes at the epicondyles of the femur. The epicondyles were identified by referencing the MRI and locating the extreme medial and lateral aspects of the femur. In Abaqus, a reference coordinate system centered on the medial epicondyle was defined. The positive $x$-axis was specified using the lateral epicondyle. A point directly inferior to the medial epicondyle was used to define the $x-y$ plane. This gave a coordinate system that matched the experimental coordinate system. The positive $\mathrm{x}$-axis was defined in the lateral direction, the positive $y$-axis was defined inferiorly, and the positive z-axis was defined in the anterior direction. The KJC was calculated by averaging the coordinates of the epicondyles then defining a reference point in Abaqus.

\subsubsection{Output Variable}

The primary output variable of interest is the cartilage contact stress, CPRESS. As mentioned previously, this is a primary indicator of cartilage damage. Other studies have also reported the contact area, CAREA, as part of their results [46], [47], [56], [61]. However, as contact stress is the value predominately responsible for cartilage degradation, contact area was judged to 
be a less significant output variable. Reported stress is the maximum value of CPRESS, averaged at the maximal node and the 18 surround nodes in the 6 neighboring elements. This averaging reduced the prominence of stress concentrations and gave a more accurate picture of the cartilage stress. In the validation study, reported stress is also averaged over a similar area to the physical sensors used experimentally. The arrangement of the 19 nodes is shown in Figure 2.5. Contact stress is reported separately for the medial and lateral sides. Each loading case will have two output results: the maximum contact pressure for each tibial compartment.

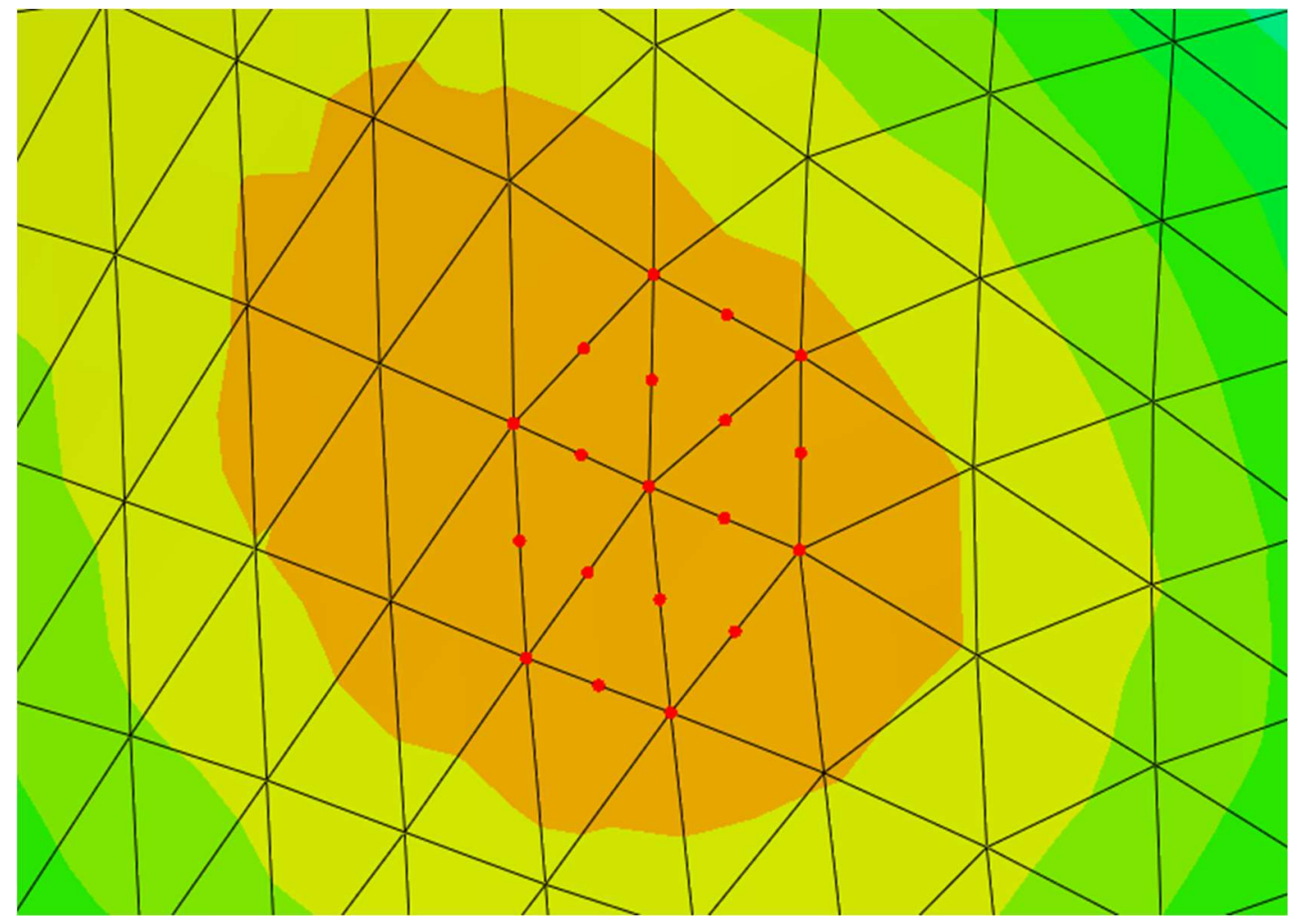

Figure 2.5 Location of the nodes used for contact pressure averaging. 


\subsubsection{Solution Steps and Boundary Conditions}

To solve the model, several discrete steps were defined. This broke the entire solution up into different regimes for various purposes. Additionally, separate boundary conditions could be applied at each step that were specific to the load being applied. Boundary conditions are critical to limiting the rigid body degrees of freedom in a FEA model. Insufficient boundary conditions will lead to rigid body translations and rotations while overly constrained models will display artificially high stiffness. Each step had Abaqus' NLGEOM flag activated to accurately capture high flexion angles and the nonlinearity introduced with contact analysis.

The first step had no applied loads or rotations and was intended to let the springs and contact definitions come to equilibrium. Because of the combination of ligament pre-stress and contact over closure, the bodies were not in a stressfree reference configuration. In the first step, sufficient freedom was given to the model to allow it to resolve the contact overclosure while the femur moved to come to equilibrium under the ligament forces. For this step, the femur had all translations and the varus-valgus rotation constrained while the flexion-extension and internal-external rotations were left free. The tibia had all rotations constrained and translations free. The varus-valgus rotation constraint on the femur was necessary to ensure model convergence in this step. Models run with the DOF left free were unable to converge. It was not immediately clear why this was the case, however this restriction was not present in subsequent steps, therefore there is no impact on the results. 
The second step was for application of a prescribed flexion angle. A prescribed rotation was specified at the KJC. This step used the same boundary conditions as the previous one, where the tibia had all rotations fixed and translations free while the femur had flexion-extension and internal-external rotations free and varus-valgus rotation and translations fixed. This allowed the femur to rotate as necessary while being stable and the tibia would translate to accommodate the contact constraints. The femur boundary conditions were modified to constrain the flexion-extension rotation to the prescribed angle.

The third step was when the load was applied. Loads and forces were applied to the KJC. To allow the model to displace adequately while remaining properly constrained, the tibia had all degrees of freedom fixed while the femur had all, except the flexion/extension angle, free. This kept the angle fixed to the prescribed value. Load step boundary conditions are shown in Figure 2.6. 


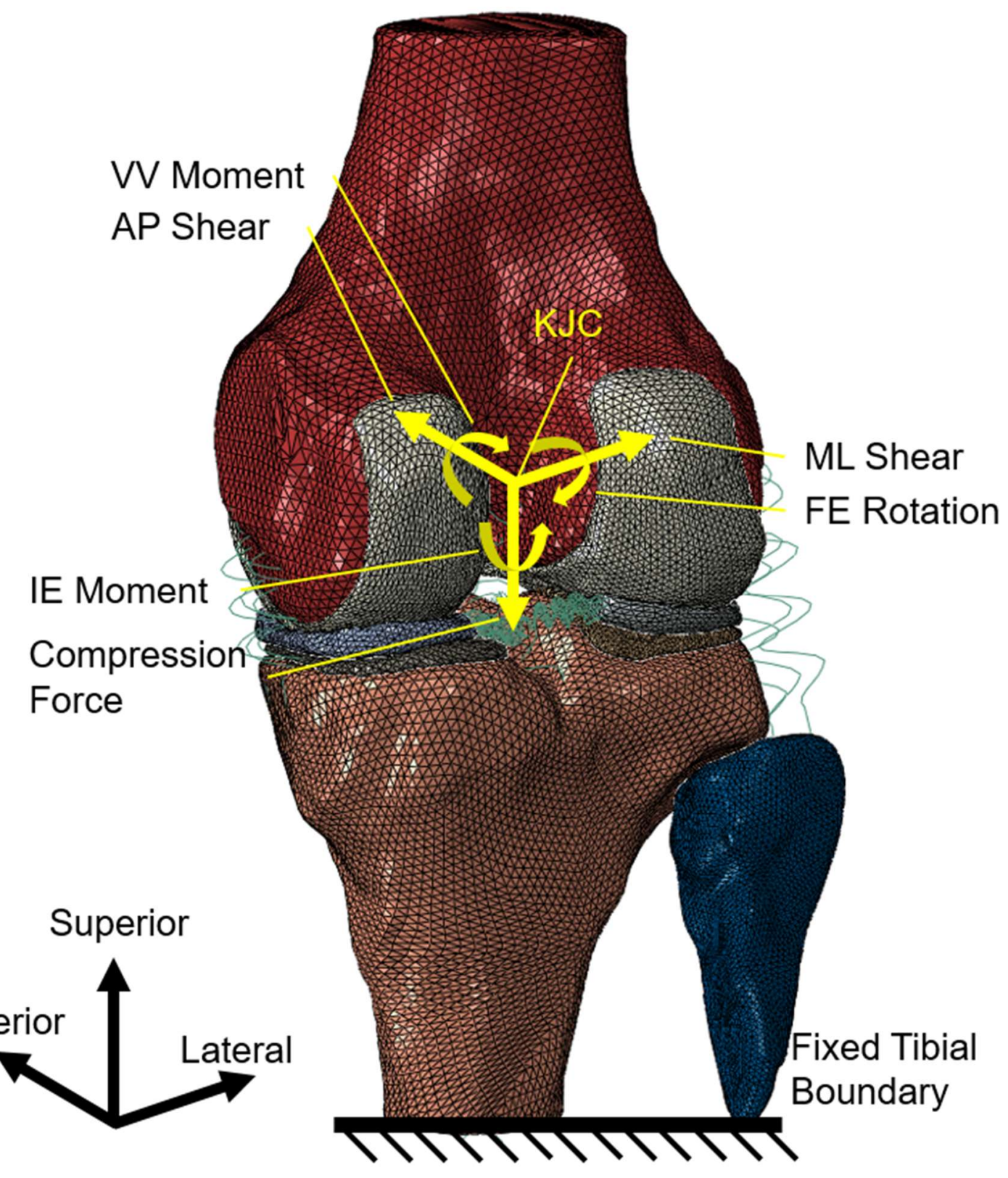

Figure 2.6 Boundary conditions and loading in the third analysis step.

\subsection{Model Convergence and Validation}

A convergence study of the model was conducted by refining the cartilaginous bodies to different levels in MeshLab by use of the IP re-meshing parameter. The menisci were not individually tested for convergence as no 
results were sampled from them. Since convergence could be obtained through the cartilage alone, increasing the degrees of freedom (DOFs) in the menisci would unnecessarily increase computational time over the entire model. Five different models ranging from 295,000 to 665,000 global DOFs were created. A $500 \mathrm{~N}$ compressive load was applied at the femur joint center and contact pressure was recorded. Because the IP filter created the surface mesh by refining larger triangles in the abstract mesh, the corner nodes of those abstract mesh faces remained in a constant location in the real domain. Since all convergence study meshes were created from the same underlying IP, this yielded a handful of nodes that were in the same location across the convergence meshes. These nodes were used to compare stress results across the models to judge convergence. In total, 15 nodes were selected, and are highlighted in Figure 2.7. 

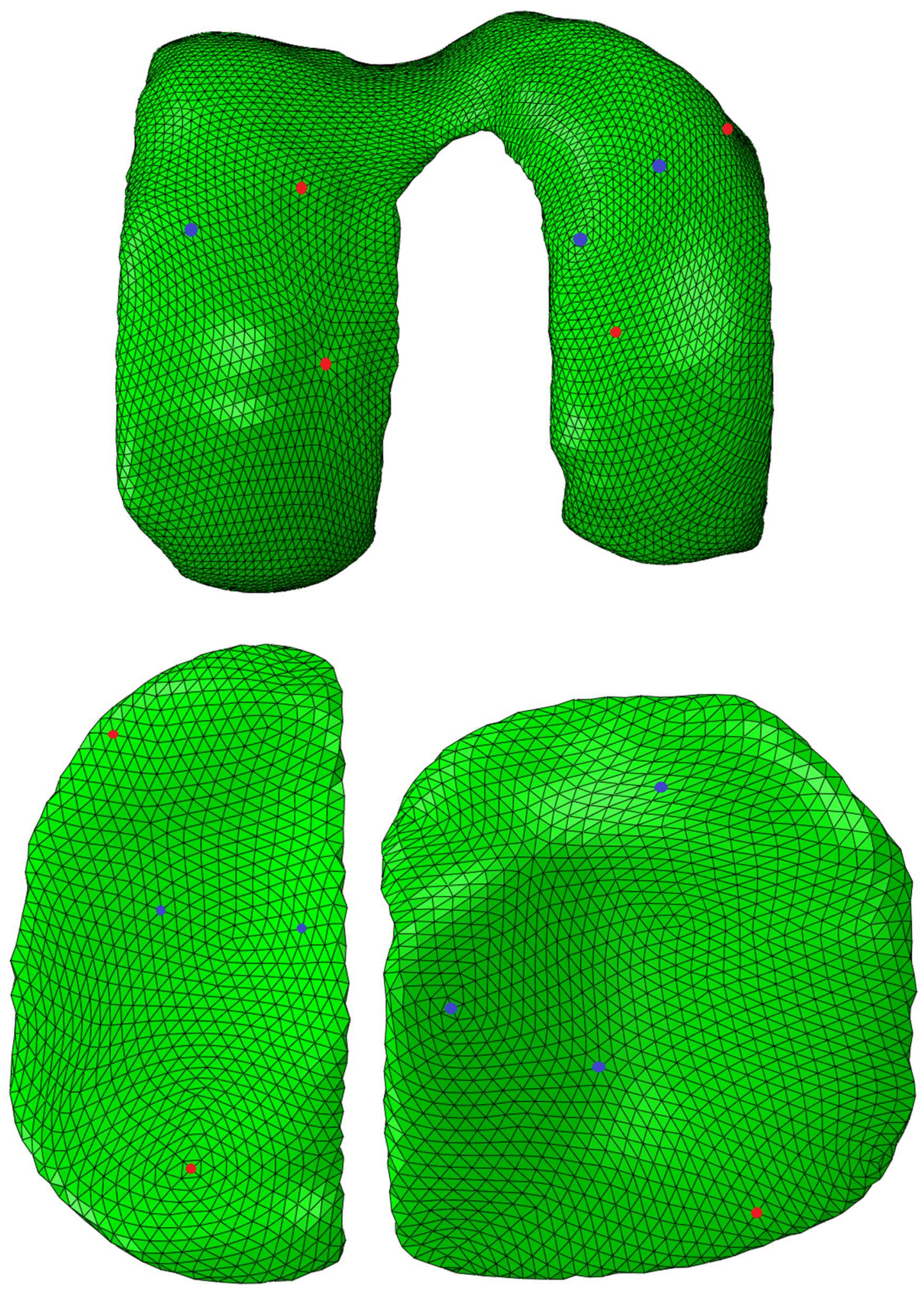

Figure 2.7 Convergence study reference nodes for the femoral cartilage (top), medial tibial cartilage (left) and lateral tibial cartilage (right). 
As expected, the models showed poor results for a low number of DOFs. Figure 2.8 shows contact stress as a function of DOFs. Some of the highlighted points in Figure 2.7 did not report any contact stress for all 5 convergence study models. The nodes that were in contact for each model are highlighted in blue in Figure 2.7. They are named in Figure 2.8 according to what body they contact (cartilage or meniscus) and where they are located relative to the other nodes. From the convergence graphs, 450,000 DOFs was selected as a target for future models to be converged. The gray vertical line shows the completed model used in this study. 

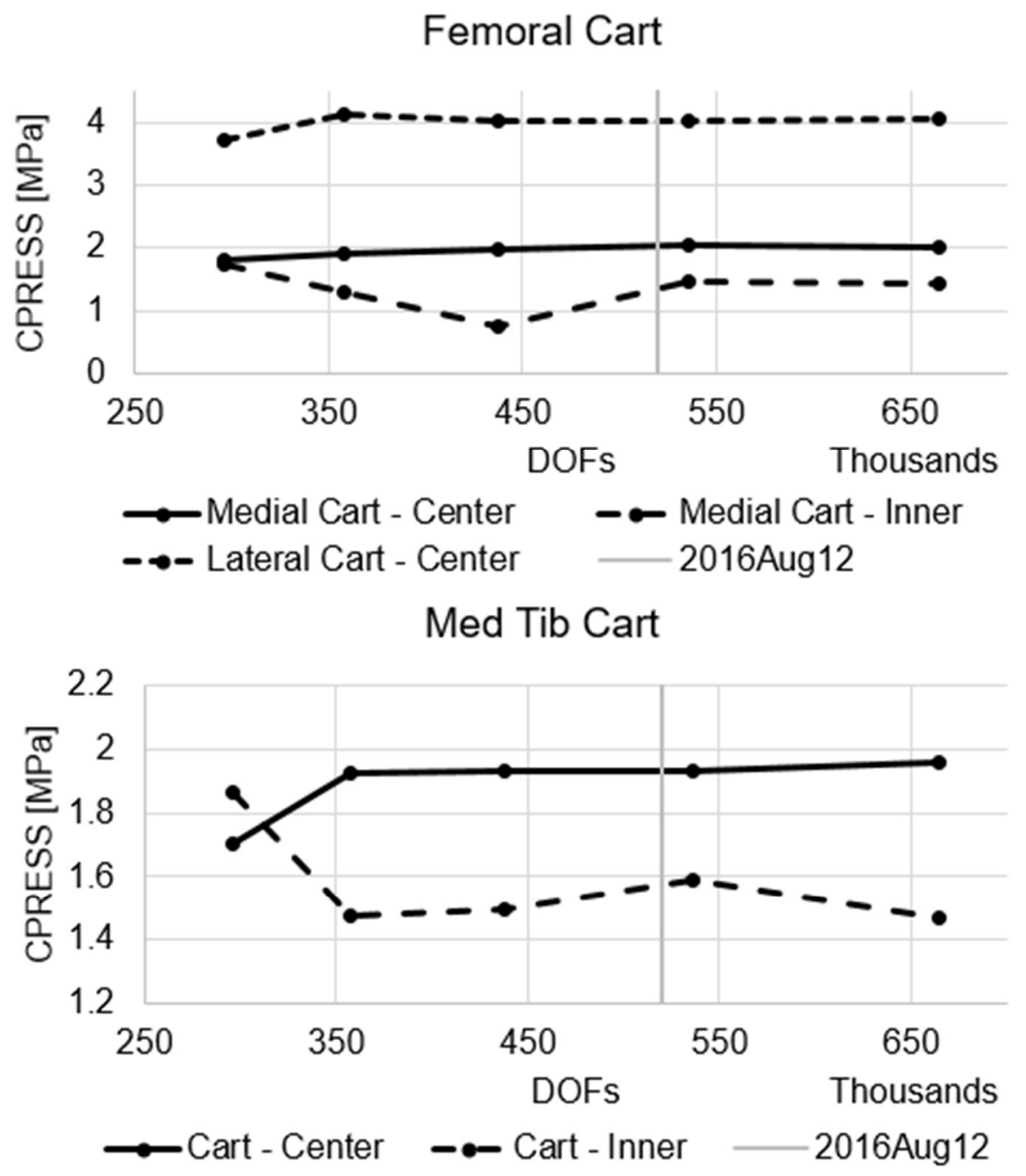

Lat Tib Cart

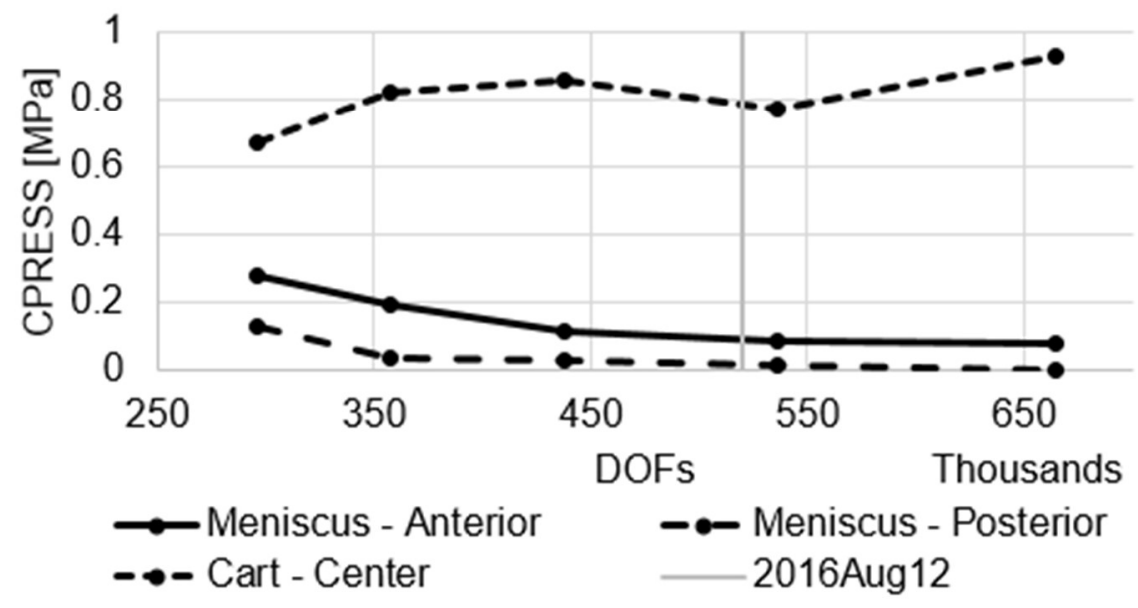

Figure 2.8 Convergence study results for the femoral cartilage (top), medial tibial cartilage (middle), and lateral tibial cartilage (bottom). 
The final model had 98,980 elements, with 517,566 DOFs. The elements per body are broken down according to Table 2.5. The meshes for the femoral cartilage, lateral tibial cartilage, and medial tibial cartilage are shown in Figure 2.9, Figure 2.10, and Figure 2.11, respectively. All three of the AC bodies are relatively thin compared to their contact area. This required a high mesh density to give a sufficient number of elements through the body thickness while preserving reasonable element aspect ratios. The meshes for the femur, fibula, and tibia are given in Figure 2.12, Figure 2.13, and Figure 2.14, respectively. The primary modeling concern was that the cartilage contact surfaces were sufficiently defined. Their overall mesh densities were a result of the density required to accurately define the contact regions. The lateral and medial menisci are shown in Figure 2.15 and Figure 2.16, respectively. These bodies become very thin in the inner annular region. Their mesh densities had to be fine enough to preserve aspect ratios in this thin section. Figure 2.17 shows the entire knee model with the femur and femoral cartilage removed to show the ligament and meniscal horn springs. The whole knee model is shown anteriorly in Figure 2.18 and posteriorly in Figure 2.19. 
Table 2.5 Number of elements in each mesh

\begin{tabular}{l|r} 
& $\begin{array}{r}\text { Number of } \\
\text { Elements }\end{array}$ \\
\hline Femoral Cartilage & 37,632 \\
Femur & 13,794 \\
Fibula & 6,912 \\
Lateral Meniscus & 5,097 \\
Lateral Tibial Cartilage & 11,897 \\
Medial Meniscus & 5,436 \\
Medial Tibial Cartialge & 5,252 \\
Tibia & 12,960 \\
\hline Total & 98,980
\end{tabular}

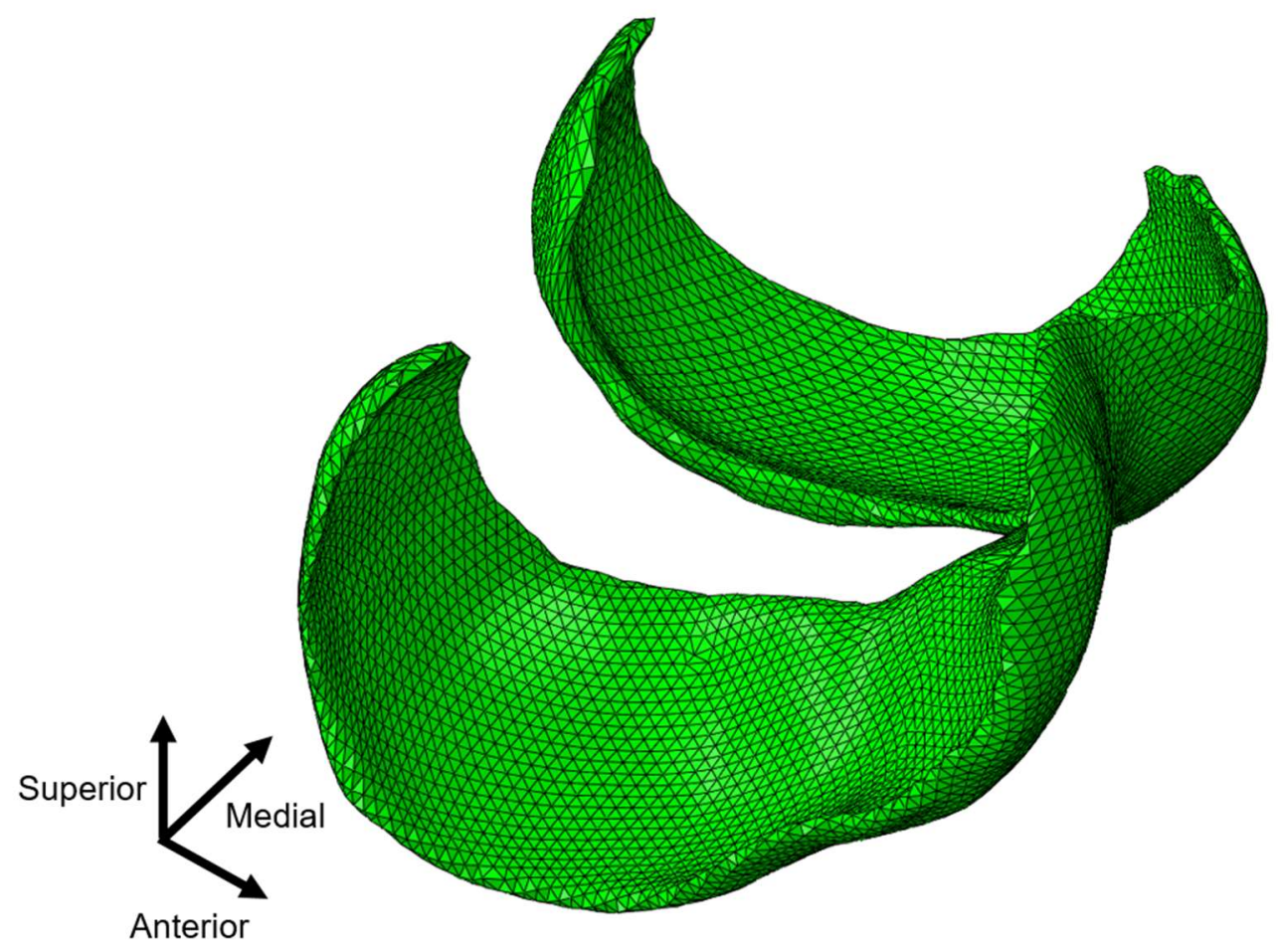

Figure 2.9 Mesh of the femoral cartilage. 


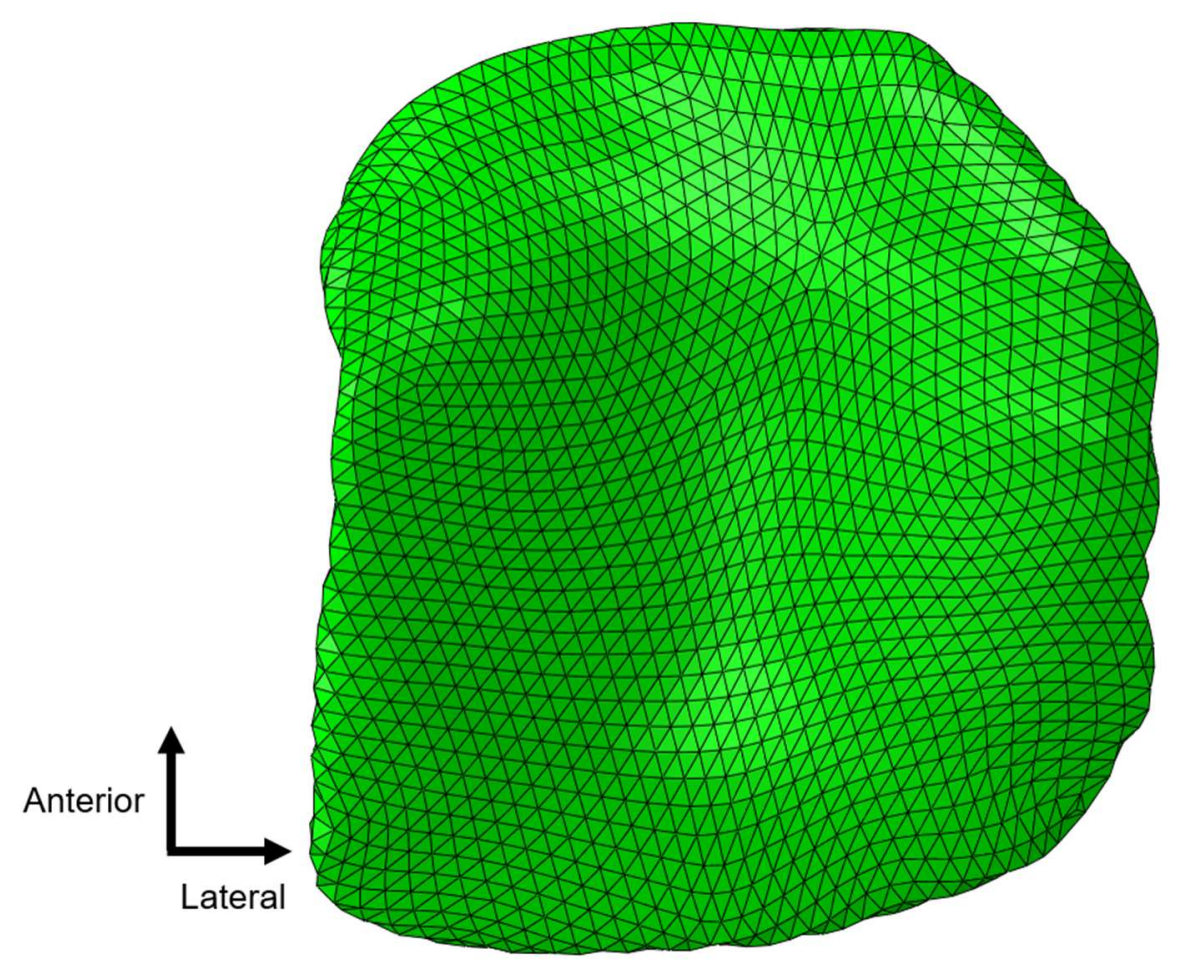

Figure 2.10 Mesh of the lateral tibial cartilage.

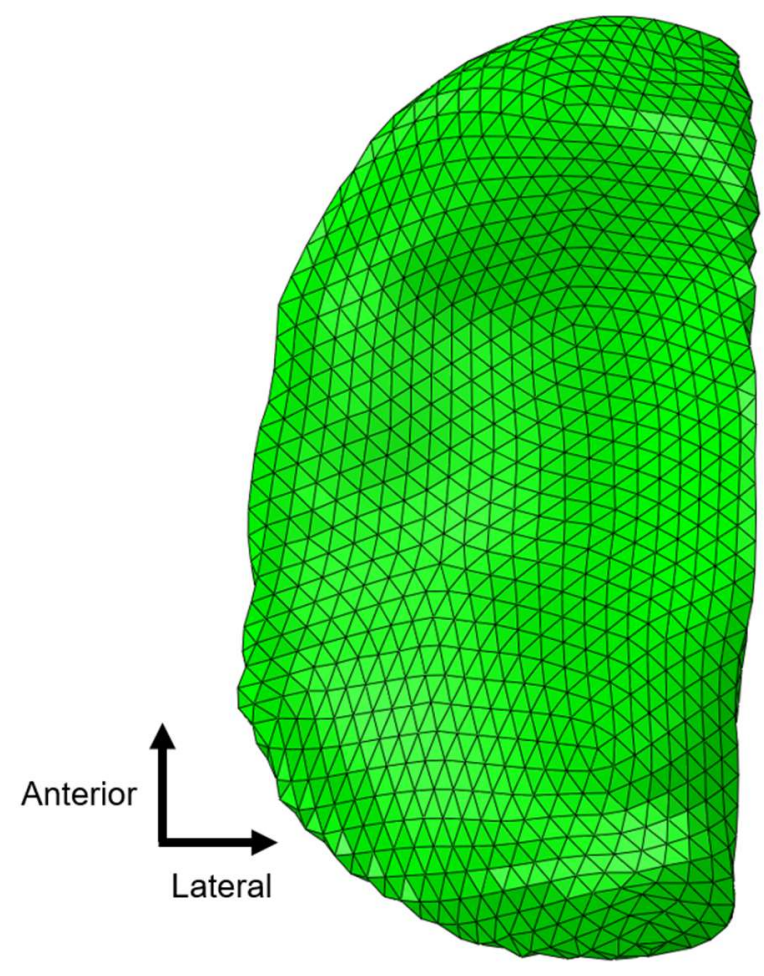

Figure 2.11 Mesh of the medial tibial cartilage. 


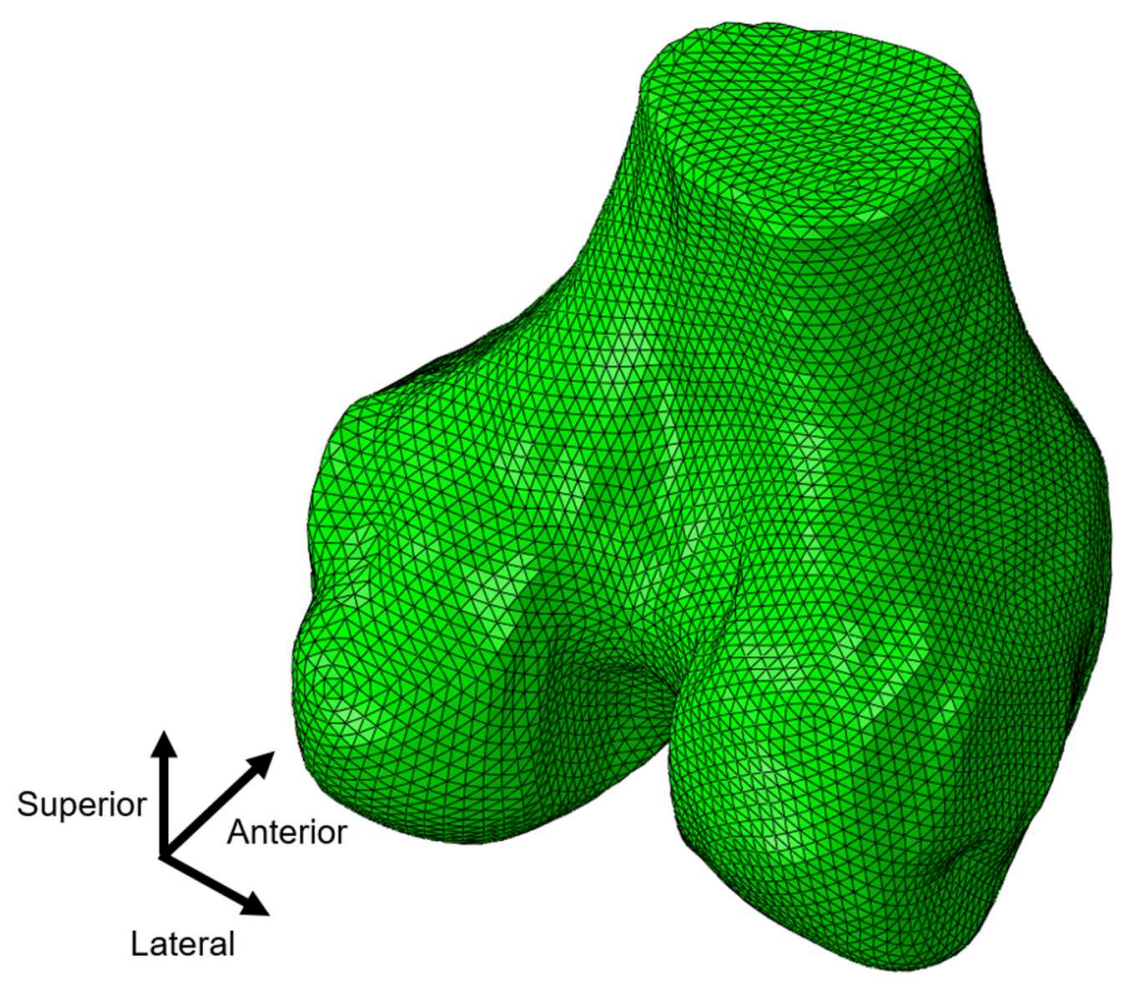

Figure 2.12 Mesh of the femur.

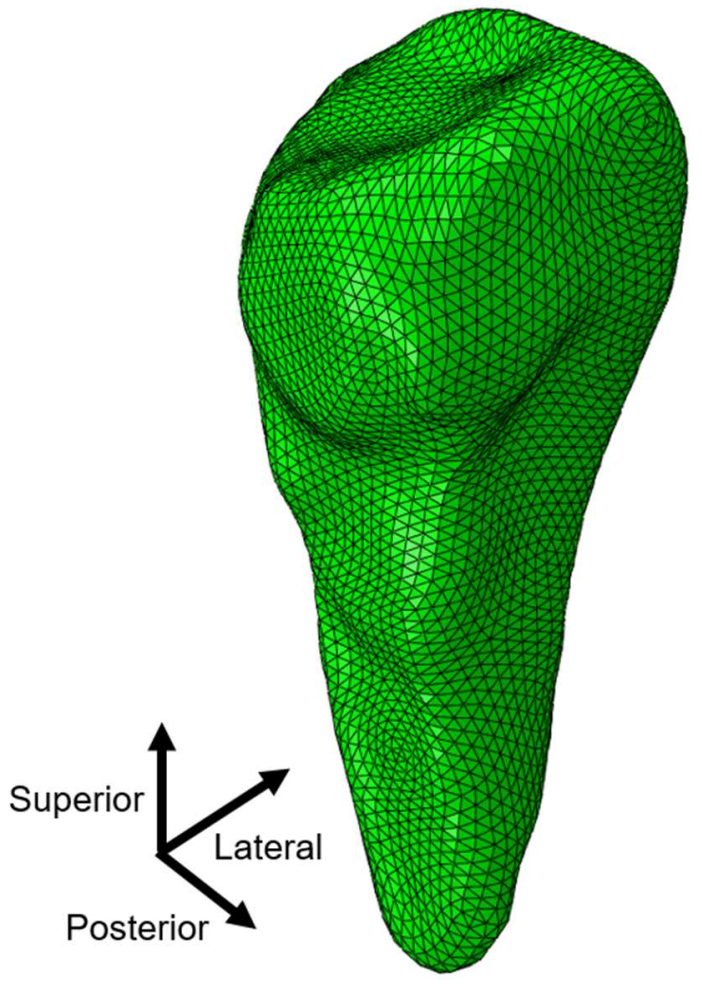

Figure 2.13 Mesh of the fibula. 


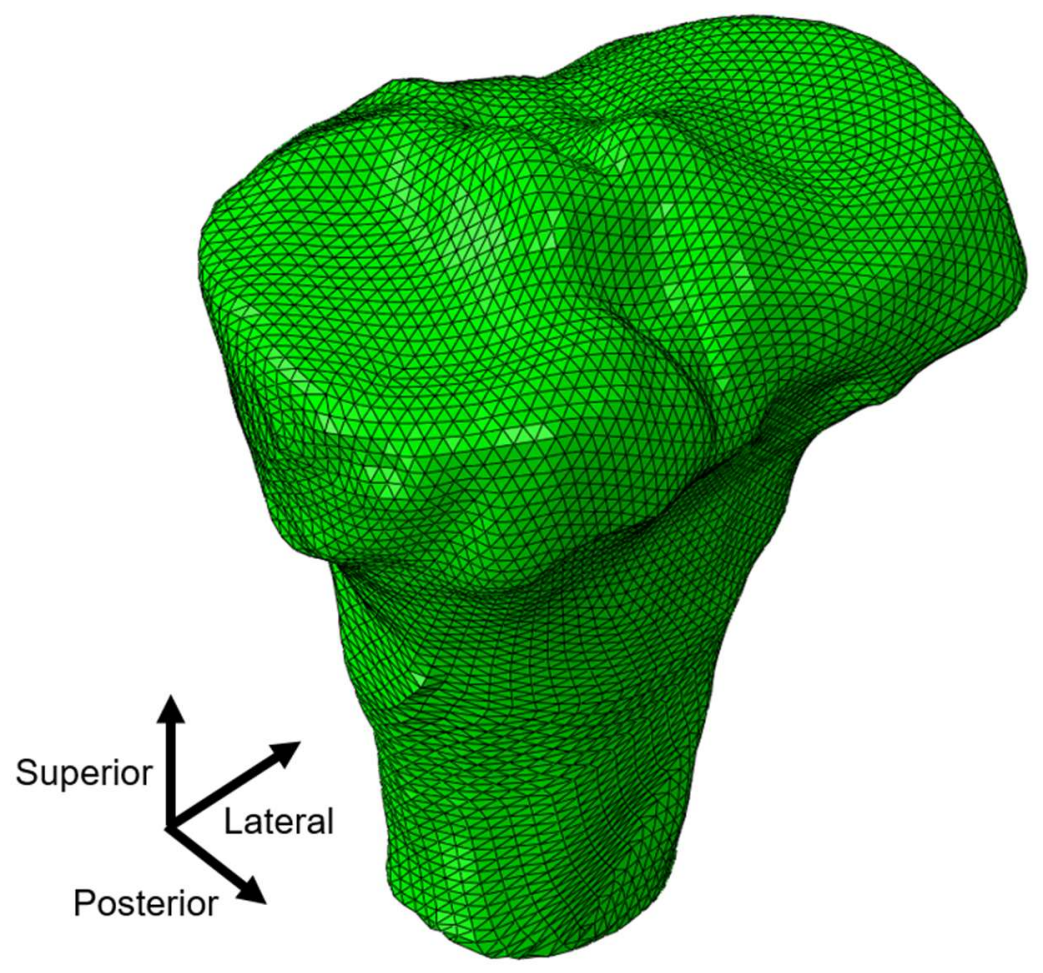

Figure 2.14 Mesh of the tibia.

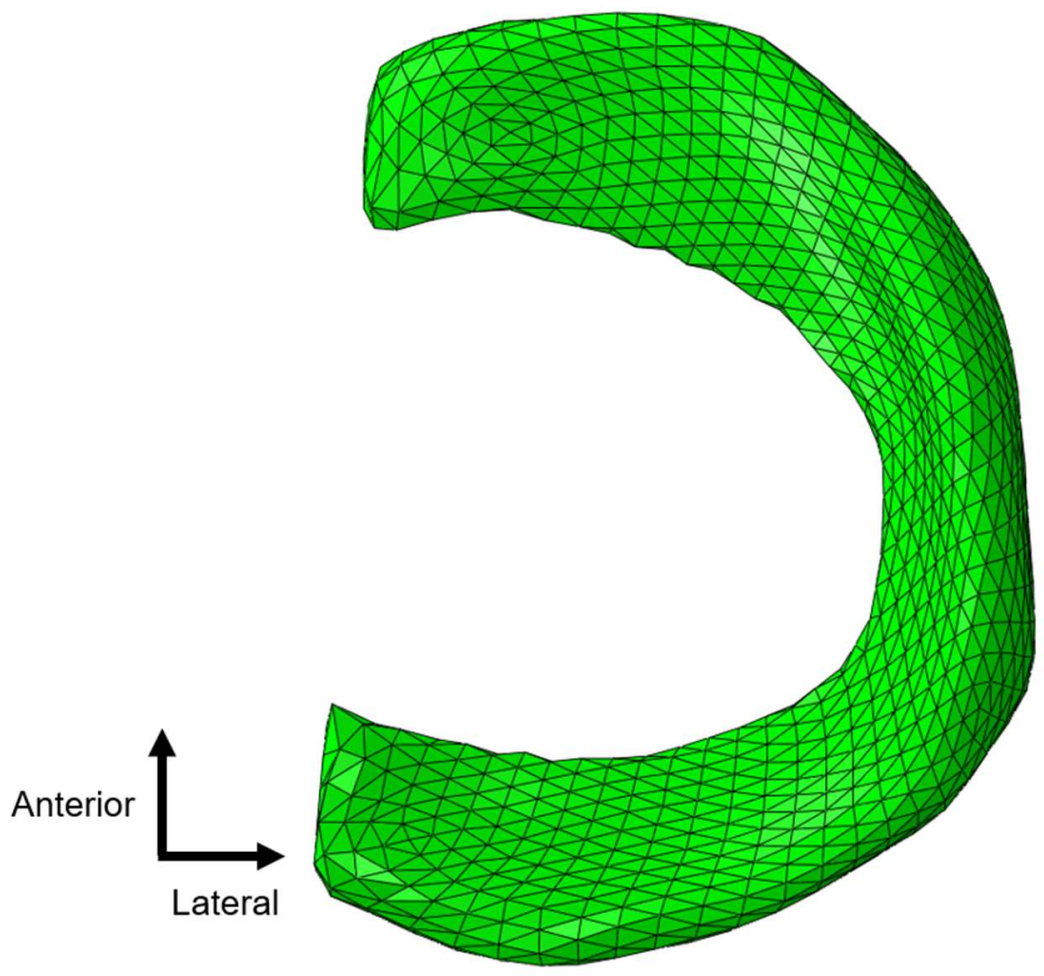

Figure 2.15 Mesh of the lateral meniscus. 


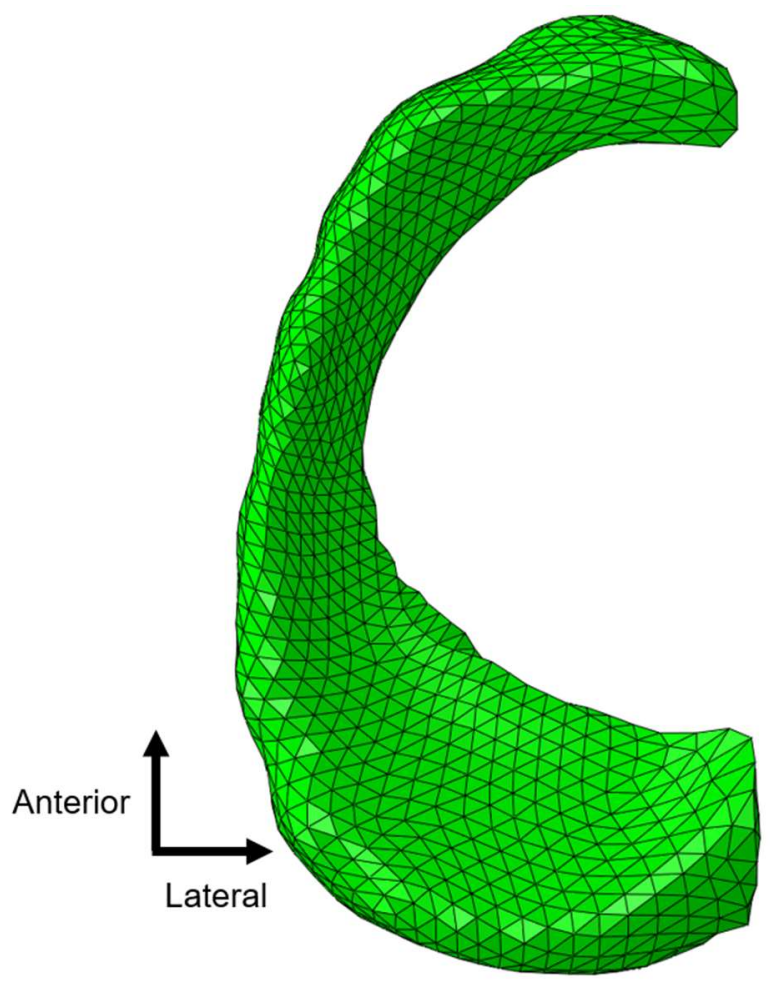

Figure 2.16 Mesh of the medial meniscus.

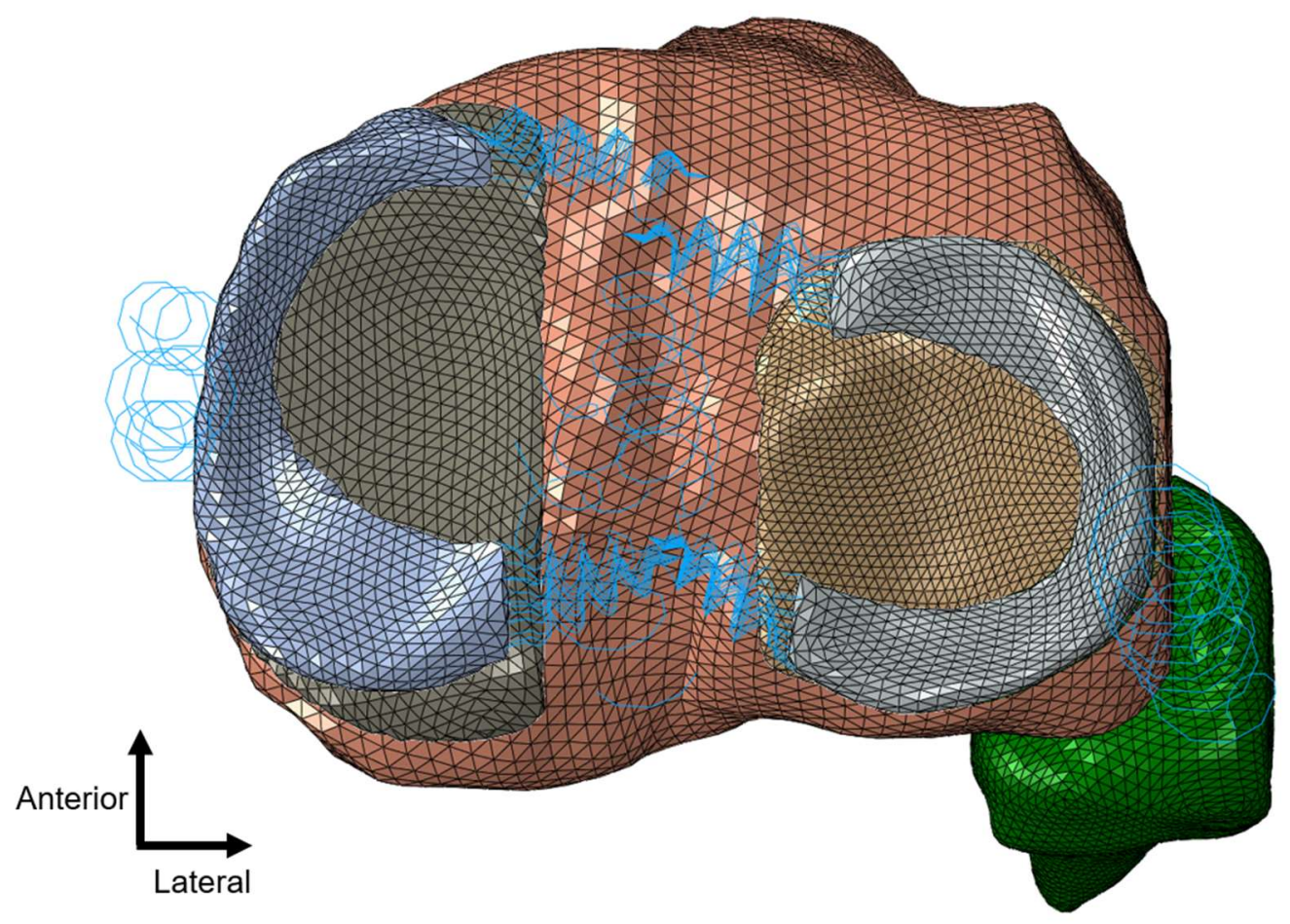

Figure 2.17 Whole knee model without femur or femoral cartilage. 


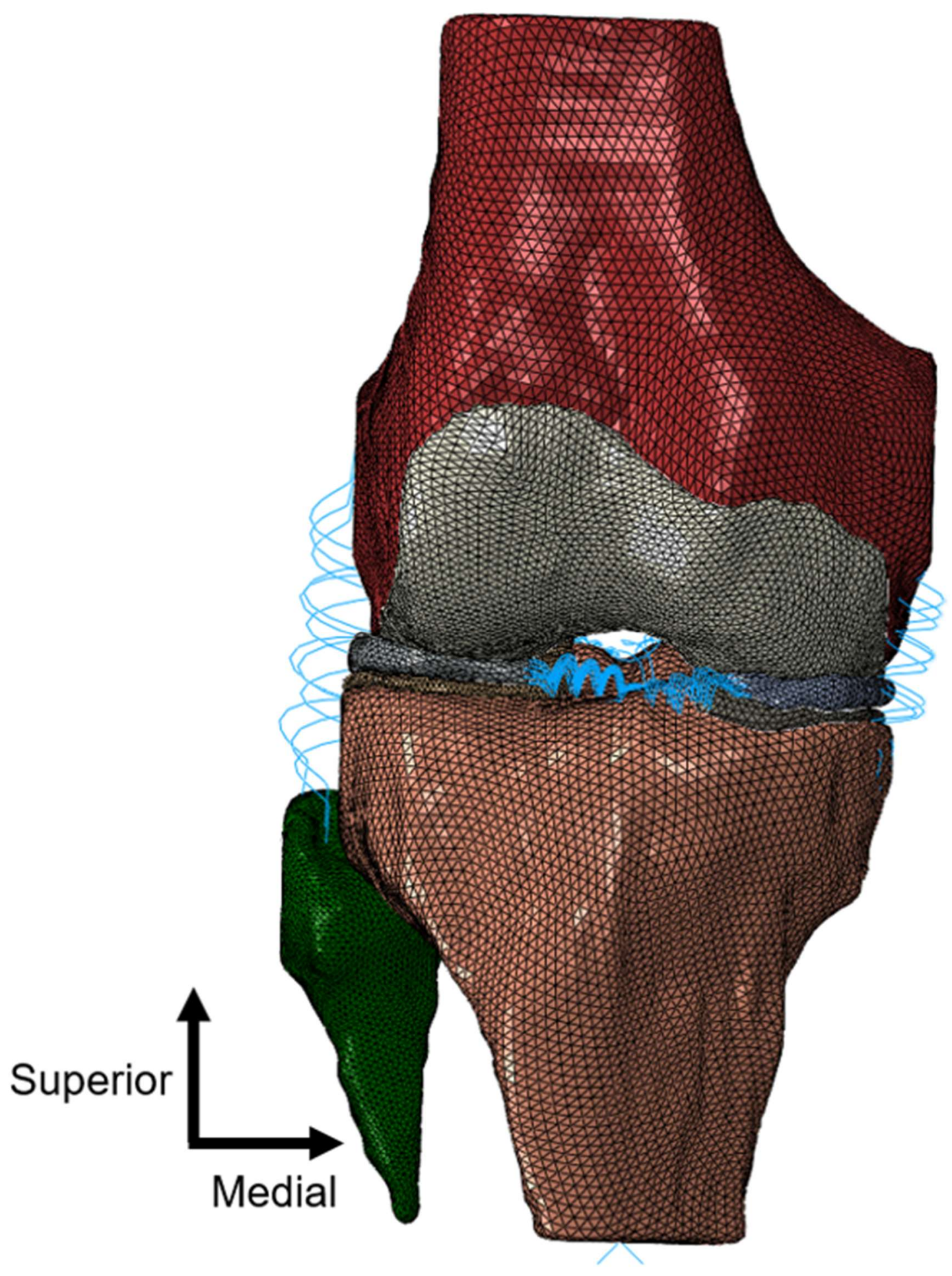

Figure 2.18 Whole knee model viewed from the anterior direction. 


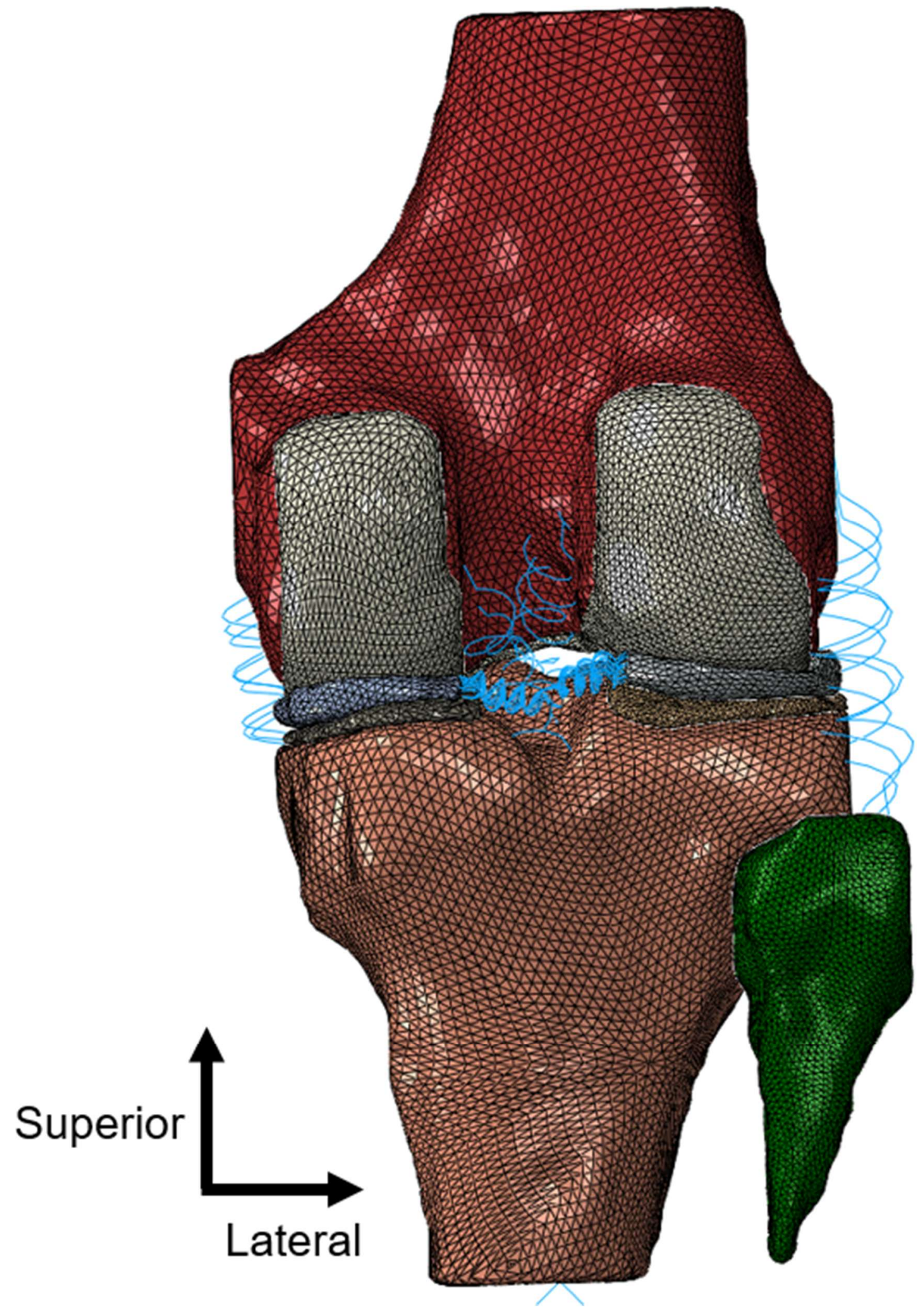

Figure 2.19 Whole knee model viewed from the posterior direction. 
The converged model was validated against published cadaveric experimental data. Seitz et. al. [62] conducted cadaver experiments investigating the changes in contact pressure in response to partial meniscectomies. The study involved inserting digital sensors between the menisci and tibial cartilage that had a spatial resolution of $1.4 \mathrm{~mm}^{2}$. The averaging routine mentioned for the cartilage contact pressure (CPRESS) averaged the FE results over a similar area. The published results for the intact meniscus were used to validate the FEA model at $500 \mathrm{~N}$ and $1000 \mathrm{~N}$ compressive loads. Boundary conditions were selected that most closely reflected those mentioned in the study. During loading, the tibia was fully constrained and the femur had flexion-extension and varusvalgus rotation fixed. Contact pressure results were measured for both the medial and lateral knee compartments on the tibial cartilage surface. Because the experimental study involved separating then reconstructing the knee joint, it is unlikely the ligaments were fully intact and providing their full levels of in situ prestress. Therefore, prestress in the ligament springs was turned off for validation studies. The models were considered validated if the CPRESS averages fell within one standard deviation of the reported mean.

\subsection{Subject Loading}

Each subject had a unique loading case specific to their average gait and cycling trials. For each gait trial, the maximum compressive load was identified and extracted, along with the anterior-posterior and medial-lateral shear, varusvalgus and internal-external rotation moments, and the flexion angle at that point 
in time. These numbers were then averaged across all trials to create an average gait load for the subject. A similar procedure was followed for cycling. Gait loads are shown in Table 2.6, cycling loads are shown in Table 2.7, while averages and standard deviations are shown in Table 2.8. Compressive, lateral, and anterior forces, as well as valgus and internal rotation moments, were defined as positive. Note that the internal moment does not strictly follow a right-handed coordinate system. This was due to a difference between the model coordinate system and the coordinate system the loads were reported in and rectified by switching the sign of the applied internal-external moment. Due to kinematic constraints of the model, flexion angles were limited to a maximum of 65 degrees. This limited some of the cycling models. Experimentally, there was not a strong interdependence between flexion angle and maximum compressive load at that point in the cycle, therefore limiting the flexion angle does not significantly alter the rest of the loading case. Additionally, one gait case, 2016Aug15-01 (marked with an * in Table 2.6 ), only completed $96.33 \%$ of the loading step before the simulation stopped. Because Abaqus was still able to converge to a solution at $96.33 \%$ of the load, that result was used along with the rest. Linearly extrapolating the data to $100 \%$ of the load does not change any of the statistical conclusions reached in the study, therefore the impact of this limitation is minimal.

A custom MATLAB script combined the loading case with the model geometry, wrote the INP file, and sent it to Abaqus. This methodology was used 
to quickly iterate various model parameters without requiring direct modification

of the INP.

\section{Table 2.6 Gait loads}

\begin{tabular}{|c|c|c|c|c|c|c|c|c|}
\hline Status & Subject ID & Leg & $\begin{array}{r}\text { Flexion } \\
\text { Angle [deg] }\end{array}$ & $\begin{array}{r}\text { Compression } \\
\text { Force }[\mathrm{N}]\end{array}$ & $\begin{array}{r}\text { A/P Shear } \\
{[\mathrm{N}]}\end{array}$ & $\begin{array}{r}\mathrm{M} / \mathrm{L} \text { Shear } \\
{[\mathrm{N}]}\end{array}$ & $\begin{array}{r}\text { VN Moment } \\
{[\mathrm{N}-\mathrm{mm}]}\end{array}$ & $\begin{array}{r}\text { le Moment } \\
{[\mathrm{N}-\mathrm{mm}]}\end{array}$ \\
\hline \multirow[t]{6}{*}{ Control } & 2016Aug12-02 & Right & 18.50 & 884.34 & 139.74 & 107.10 & 31488.74 & -26.56 \\
\hline & 2016Aug19-01 & Left & 16.96 & 968.58 & 67.26 & 42.69 & 12698.74 & 911.33 \\
\hline & 2016Nov05-01 & Right & 16.44 & 599.51 & 102.73 & 27.13 & 11236.60 & 4750.14 \\
\hline & 2016Aug15-01* & Right & 27.90 & 1018.59 & 62.27 & 52075.34 & 40153.95 & 2141.85 \\
\hline & 2016Aug26-01 & Right & 8.40 & 822.52 & 51.86 & 280.49 & 21888.62 & 11092.81 \\
\hline & 2016Sep01-01 & Left & 21.79 & 869.09 & 32.66 & 190.28 & 10894.58 & 769.41 \\
\hline \multirow[t]{6}{*}{ Amputee } & 2016Nov10-01 & Left & 23.14 & 842.30 & 138.90 & 90.10 & 29210.44 & 3218.48 \\
\hline & 2016 Nov14-02 & Right & 22.35 & 833.24 & 209.51 & -0.44 & -3284.85 & -1602.99 \\
\hline & 2017Mar18-01 & Right & 22.05 & 897.69 & 160.97 & 55.98 & 7260.81 & -578.23 \\
\hline & 2017Nov11-01 & Left & 25.32 & 954.12 & 100.82 & 214.49 & 45942.29 & -720.24 \\
\hline & 20170ct30-01 & Right & 23.77 & 763.11 & -2.20 & 174.87 & -858.52 & -4019.67 \\
\hline & 2017Sep09-01 & Right & 11.36 & 824.65 & 92.36 & 312.16 & 26109.82 & 7402.04 \\
\hline
\end{tabular}

\section{Table 2.7 Cycling loads}

\begin{tabular}{|c|c|c|c|c|c|c|c|c|}
\hline Status & Subject ID & Leg & $\begin{array}{r}\text { Flexion } \\
\text { Angle [deg] }\end{array}$ & $\begin{array}{r}\text { Compression } \\
\text { Force }[\mathrm{N}] \\
\end{array}$ & $\begin{array}{r}\text { A/P Shear } \\
{[\mathrm{N}]}\end{array}$ & $\begin{array}{r}\text { M/L Shear } \\
{[\mathrm{N}]}\end{array}$ & $\begin{array}{r}\text { VN Moment } \\
{[\mathrm{N}-\mathrm{mm}]}\end{array}$ & $\begin{array}{r}\text { l/E Moment } \\
{[\mathrm{N}-\mathrm{mm}]}\end{array}$ \\
\hline \multirow[t]{6}{*}{ Control } & 2016Aug12-02 & Right & 63.98 & 102.43 & 26.87 & 13.43 & -3.63 & -564.79 \\
\hline & 2016Aug19-01 & Left & 65.00 & 78.54 & 12.63 & 13.23 & 3665.08 & -1665.46 \\
\hline & 2016Nov05-01 & Right & 49.03 & 53.56 & 8.23 & 0.11 & -1093.59 & 693.99 \\
\hline & 2016Aug15-01 & Right & 64.14 & 70.76 & 3.12 & 659.73 & -1488.65 & 124.18 \\
\hline & 2016Aug26-01 & Right & 65.00 & 75.07 & -2.85 & 22.40 & -3694.08 & -502.79 \\
\hline & 2016Sep01-01 & Left & 39.13 & 64.35 & 25.64 & 11.96 & 11160.25 & 1778.26 \\
\hline \multirow[t]{6}{*}{ Amputee } & 2016Nov10-01 & Left & 65.00 & 39.86 & 14.69 & 3.85 & 988.48 & -534.79 \\
\hline & 2016Nov14-02 & Right & 65.00 & 62.91 & 25.80 & 4.66 & -1284.27 & 407.83 \\
\hline & 2017Mar18-01 & Right & 65.00 & 76.23 & -57.58 & -2.28 & 2614.85 & -1114.02 \\
\hline & 2017Nov11-01 & Left & 65.00 & 77.98 & 2.36 & 15.66 & -1285.05 & -543.00 \\
\hline & 2017 Oct $30-01$ & Right & 65.00 & 57.77 & 0.14 & 11.92 & -2028.84 & 224.55 \\
\hline & 2017Sep09-01 & Right & 38.62 & 61.92 & 11.94 & 14.00 & 3244.35 & 1930.85 \\
\hline
\end{tabular}


Table 2.8 Summarized loads

\begin{tabular}{|c|c|c|c|c|c|c|c|c|}
\hline Exercise & Status & & $\begin{array}{r}\text { Flexion } \\
\text { Angle [deg] }\end{array}$ & $\begin{array}{r}\text { Compression } \\
\text { Force }[\mathrm{N}] \\
\end{array}$ & $\begin{array}{r}\text { A/P Shear } \\
{[\mathrm{N}]}\end{array}$ & $\begin{array}{r}M / L \text { Shear } \\
{[N]}\end{array}$ & $\begin{array}{r}\text { VN Moment } \\
{[\mathrm{N}-\mathrm{mm}]}\end{array}$ & $\begin{array}{r}\text { I/E Moment } \\
\text { [N-mm] }\end{array}$ \\
\hline \multirow[t]{4}{*}{ Gait } & \multirow[t]{2}{*}{ Control } & Mean & 17.32 & 836.43 & 166.81 & 66.23 & 23252.73 & 3817.35 \\
\hline & & Std. Dev. & 4.94 & 125.38 & 78.86 & 44.60 & 15908.18 & 4215.20 \\
\hline & \multirow[t]{2}{*}{ Amputee } & Mean & 21.33 & 852.52 & 201.82 & 56.10 & 17396.67 & 616.57 \\
\hline & & Std. Dev. & 5.02 & 65.74 & 61.23 & 47.05 & 19463.04 & 4060.75 \\
\hline \multirow[t]{4}{*}{ Cycling } & \multirow[t]{2}{*}{ Control } & Mean & 57.71 & 74.12 & 123.64 & 8.78 & 1424.23 & -22.77 \\
\hline & & Std. Dev. & 11.02 & 16.44 & 262.72 & 10.66 & 5345.50 & 1183.36 \\
\hline & \multirow[t]{2}{*}{ Amputee } & Mean & 60.60 & 62.78 & 4.08 & 3.44 & 374.92 & 61.90 \\
\hline & & Std. Dev. & 10.77 & 13.88 & 30.60 & 4.87 & 2232.21 & 1071.80 \\
\hline
\end{tabular}

\subsection{Statistics}

Once all 24 jobs were run, maximum contact pressures were extracted and averaged. There were 4 averages to compare for each of the 2 knee compartments (medial, lateral): 2 subject conditions (control, amputee) with 2 exercises (gait, cycling). Because the study was not interested in pressure differences across the knee, the medial and lateral values were considered independently. For each compartment of the knee, 2-way ANOVA $(\alpha=0.05)$ determined the presence of any significant interactions. For all interactions deemed significant, a post-hoc Tukey pairwise comparison $(p<0.05$ significant $)$ found if the difference was statistically significant.

Additionally, to correct for any effects of body mass, height, or BMI, the contact pressure results were normalized by body weight (BW), BW divided by height $(\mathrm{BWH})$, and $\mathrm{BW}$ divided by height squared $(\mathrm{BWH} 2)$. This yielded four sets of results to be analyzed. The statistical results in each data set were assessed for trends between the normalization schemes. 


\section{RESULTS}

\subsection{Validation Results}

The results from both the $500 \mathrm{~N}$ and $1000 \mathrm{~N}$ compressive load validation studies are shown in Table 3.1. The FE model validated well against the experimental study, with each compartment being within one standard deviation of the experimental mean. This indicates that the geometry and material modeling reflect realistic physiology to a reasonable degree. However, due to how the experiment was conducted and the fact that ligaments were likely not included, this does not validate our ligament methodology. That said, because the ligaments are supported with literature, it is reasonable to accept the FEA results with them included.

Table 3.1 Validation study results

\begin{tabular}{|c|c|c|c|c|c|}
\hline & \multicolumn{4}{|c|}{ Seitz $-500 \mathrm{~N}$} & \multirow{2}{*}{ FEA } \\
\hline [MPa] & Mean-SD & Mean & Std. Dev & Mean+SD & \\
\hline Medial & 1.18 & 1.78 & 0.60 & 2.38 & 1.273 \\
\hline Lateral & 0.97 & 1.79 & 0.82 & 2.61 & 1.826 \\
\hline
\end{tabular}

\begin{tabular}{|c|c|c|c|c|c|}
\hline & \multicolumn{4}{|c|}{ Seitz $-1000 \mathrm{~N}$} & \multirow{2}{*}{ FEA } \\
\hline MPa] & Mean-SD & Mean & Std. Dev. & Mean+SD & \\
\hline Medial & 1.95 & 2.82 & 0.87 & 3.69 & 2.147 \\
\hline Lateral & 1.72 & 3.04 & 1.32 & 4.36 & 3.213 \\
\hline
\end{tabular}

\subsection{Subject Results}

The results from the 24 loading cases are shown in Figure 3.1. Individual contour plots for each model can be found in Appendix A. Actual and normalized 
values are listed in Table 3.2 and summarized in Table 3.3 for gait as well as Table 3.4 and Table 3.5 for cycling. For each subject, the medial results were higher than the lateral in both exercises and for both subject types. Additionally, the standard deviation for each group was largest in medial gait for both amputees (5.957 MPa) and controls (5.789 MPa). For each normalization scheme, group standard deviations relative to their respective average did not significantly change.

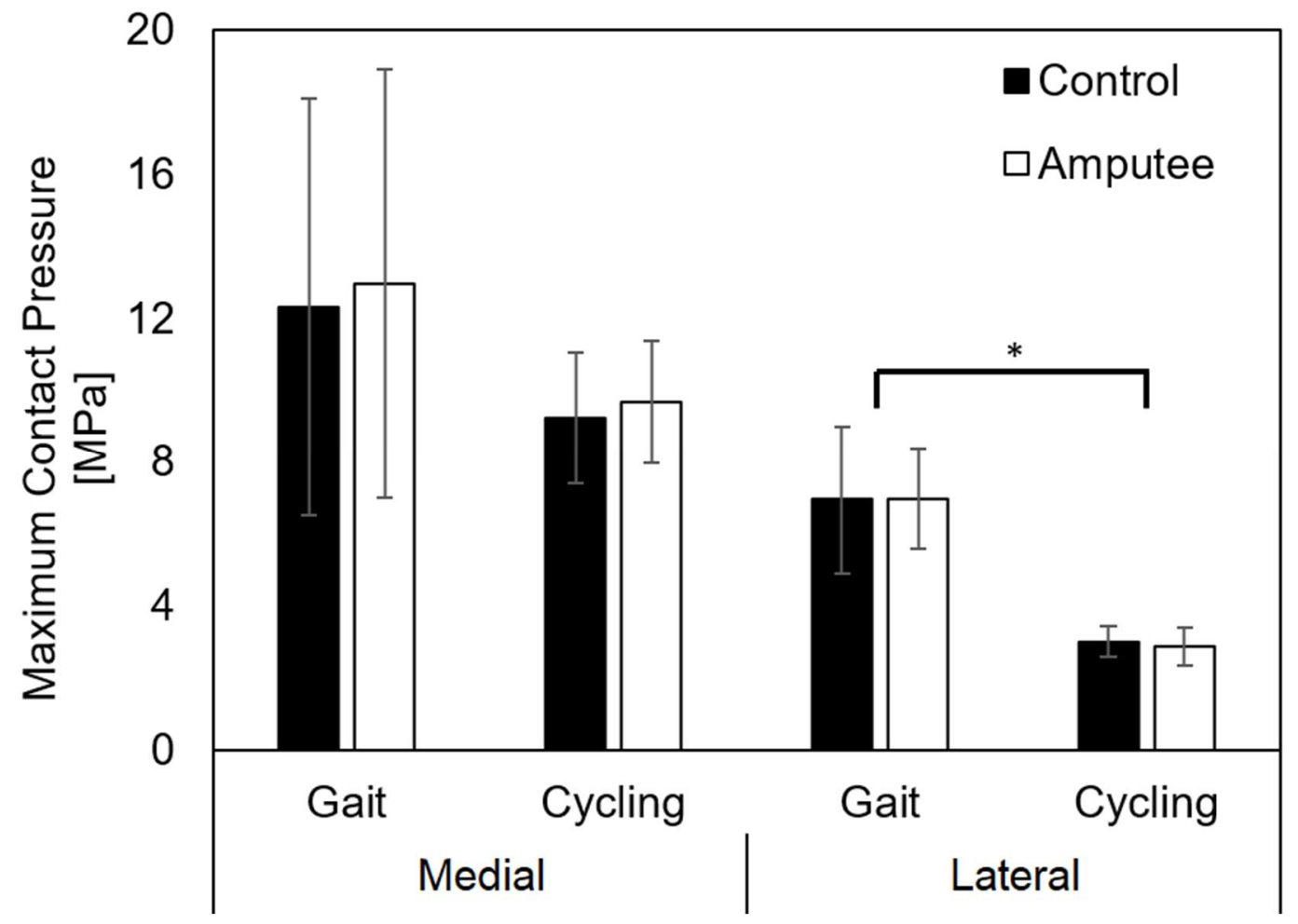

Figure 3.1 Summarized maximum contact pressure results without normalization ( ${ }^{*}$ indicates significance, $p<0.05$ ) 
Table 3.2 Actual and normalized gait maximum cartilage contact pressure [MPa]

\begin{tabular}{l|rr|rr|rr|rr} 
Normalization & \multicolumn{2}{|c|}{ None } & \multicolumn{2}{c|}{ BW } & \multicolumn{2}{c|}{ BWH } & \multicolumn{2}{c}{ BWH2 } \\
\hline Status & Medial & Lateral & Medial & Lateral & Medial & Lateral & Medial & Lateral \\
\hline Control & 9.899 & 7.981 & 0.0122 & 0.00986 & 0.0222 & 0.0179 & 0.0403 & 0.0325 \\
& 8.672 & 4.919 & 0.0100 & 0.00567 & 0.0175 & 0.00992 & 0.0306 & 0.0174 \\
& 6.832 & 5.565 & 0.0114 & 0.00926 & 0.0204 & 0.0166 & 0.0364 & 0.0297 \\
& 19.513 & 9.423 & 0.0230 & 0.0111 & 0.0411 & 0.0198 & 0.0736 & 0.0355 \\
& 19.814 & 8.885 & 0.0263 & 0.0118 & 0.0451 & 0.0202 & 0.0770 & 0.0345 \\
& 9.099 & 4.908 & 0.0112 & 0.00606 & 0.0208 & 0.0112 & 0.0385 & 0.0207 \\
\hline Amputee & 10.135 & 7.011 & 0.0138 & 0.00955 & 0.0251 & 0.0174 & 0.0457 & 0.0316 \\
& 8.048 & 6.020 & 0.00978 & 0.00731 & 0.0165 & 0.0124 & 0.0279 & 0.0209 \\
& 9.463 & 5.039 & 0.0119 & 0.00636 & 0.0216 & 0.0115 & 0.0391 & 0.0208 \\
& 15.323 & 8.192 & 0.0169 & 0.00906 & 0.0299 & 0.0160 & 0.0528 & 0.0282 \\
& 10.683 & 6.767 & 0.0151 & 0.00953 & 0.0271 & 0.0172 & 0.0488 & 0.0309 \\
& 24.020 & 8.837 & 0.0304 & 0.0112 & 0.0541 & 0.0199 & 0.0963 & 0.0354
\end{tabular}

Table 3.3 Actual and normalized gait maximum cartilage contact pressure, averaged [MPa]

\begin{tabular}{|c|c|c|c|c|c|c|c|c|c|}
\hline \multicolumn{2}{|c|}{ Normalization } & \multicolumn{2}{|c|}{ None } & \multicolumn{2}{|c|}{ BW } & \multicolumn{2}{|c|}{ BWH } & \multicolumn{2}{|c|}{$\mathrm{BWH} 2$} \\
\hline Status & Side & Mean & $\mathrm{SD}$ & Mean & $\mathrm{SD}$ & Mean & SD & Mean & SD \\
\hline \multirow[t]{2}{*}{ Control } & Medial & 12.305 & 5.789 & 0.0157 & 0.00706 & 0.0278 & 0.0120 & 0.0494 & 0.0204 \\
\hline & Lateral & 6.947 & 2.056 & 0.00896 & 0.00256 & 0.0159 & 0.00439 & 0.0284 & 0.00758 \\
\hline \multirow[t]{2}{*}{ Amputee } & Medial & 12.945 & 5.957 & 0.0163 & 0.00732 & 0.0291 & 0.0131 & 0.0518 & 0.0235 \\
\hline & Lateral & 6.977 & 1.390 & 0.00883 & 0.00173 & 0.0157 & 0.00320 & 0.0280 & 0.00597 \\
\hline
\end{tabular}

Table 3.4 Actual and normalized cycling maximum cartilage contact pressure [MPa]

\begin{tabular}{l|rr|rr|rr|rr} 
Normalization & \multicolumn{2}{|c|}{ None } & \multicolumn{2}{c|}{ BW } & \multicolumn{2}{c|}{ BWH } & \multicolumn{2}{c}{ BWH2 } \\
\hline Status & Medial & Lateral & Medial & Lateral & Medial & Lateral & Medial & Lateral \\
\hline Control & 10.222 & 3.248 & 0.0126 & 0.00401 & 0.0229 & 0.0132 & 0.0403 & 0.0416 \\
& 10.085 & 3.526 & 0.0116 & 0.00406 & 0.0203 & 0.0124 & 0.0306 & 0.0356 \\
& 7.844 & 3.270 & 0.0131 & 0.00544 & 0.0234 & 0.0174 & 0.0364 & 0.0418 \\
& 10.451 & 3.062 & 0.0123 & 0.00360 & 0.0220 & 0.0115 & 0.0736 & 0.0394 \\
& 10.624 & 2.510 & 0.0141 & 0.00334 & 0.0242 & 0.00976 & 0.0770 & 0.0413 \\
& 6.127 & 2.440 & 0.0076 & 0.00301 & 0.0140 & 0.0103 & 0.0385 & 0.0259 \\
\hline Amputee & 10.199 & 3.301 & 0.0139 & 0.00450 & 0.0253 & 0.0149 & 0.0457 & 0.0460 \\
& 10.569 & 2.700 & 0.0128 & 0.00328 & 0.0217 & 0.00937 & 0.0279 & 0.0367 \\
& 10.172 & 3.596 & 0.0128 & 0.00454 & 0.0232 & 0.0149 & 0.0391 & 0.0421 \\
& 10.407 & 2.879 & 0.0115 & 0.00318 & 0.0203 & 0.00991 & 0.0528 & 0.0358 \\
& 10.518 & 2.527 & 0.0148 & 0.00356 & 0.0267 & 0.0115 & 0.0488 & 0.0480 \\
& 6.235 & 2.173 & 0.0079 & 0.00275 & 0.0140 & 0.00871 & 0.0963 & 0.0250
\end{tabular}


Table 3.5 Actual and normalized cycling maximum cartilage contact pressure, averaged $[\mathrm{MPa}]$

\begin{tabular}{ll|rr|rr|rr|rr}
\multicolumn{2}{l|}{ Normalization } & \multicolumn{2}{|c|}{ None } & \multicolumn{2}{|c|}{ BW } & \multicolumn{2}{|c|}{ BWH } & \multicolumn{2}{|c}{ BWH2 } \\
\hline Status & Side & Mean & SD & Mean & SD & Mean & SD & Mean & SD \\
\hline \multirow{2}{*}{ Control } & Medial & 9.226 & 1.827 & 0.0119 & 0.00227 & 0.0211 & 0.00373 & 0.0376 & 0.00620 \\
& Lateral & 3.009 & 0.440 & 0.00391 & 0.000850 & 0.00698 & 0.00152 & 0.0125 & 0.00276 \\
\hline Amputee & Medial & 9.684 & 1.697 & 0.0123 & 0.00243 & 0.0219 & 0.00448 & 0.0389 & 0.00839 \\
& Lateral & 2.863 & 0.519 & 0.00363 & 0.00073 & 0.0065 & 0.00142 & 0.0115 & 0.00275
\end{tabular}

\subsection{Statistics}

ANOVA statistical results for each normalization scheme and interaction

are shown in Table 3.6. While there were slight variations in $p$-values due to normalization scheme, there were no changes in significance. Because normalization had no impact on significance, only the non-normalized values will be discussed. Conclusions drawn from the non-normalized values can be similarly drawn from the normalized groups.

Table 3.6 ANOVA statistical results ( ${ }^{*}$ indicates a significant result, $p<0.05$ )

\begin{tabular}{ll|rc} 
& & \multicolumn{2}{|c}{ P-Value } \\
Normalization & Interaction & Medial & Lateral \\
\hline None & Exercise & 0.088 & $<0.0001^{*}$ \\
& Status & 0.760 & 0.913 \\
& Exercise $^{*}$ Status & 0.959 & 0.868 \\
\hline BW & Exercise & 0.088 & $<0.0001^{*}$ \\
& Status & 0.814 & 0.766 \\
& Exercise Status & 0.962 & $0.912^{*}$ \\
\hline BWH & Exercise & 0.084 & $<0.0001^{*}$ \\
& Status & 0.799 & 0.762 \\
& Exercise*Status & 0.950 & 0.910 \\
\hline BWH2 & Exercise & 0.081 & $<0.0001{ }^{*}$ \\
& Status & 0.786 & 0.759 \\
& Exercise & \\
& & 0.939 & 0.909
\end{tabular}


In the medial compartment, there was no significant dependence on exercise $(p=0.088)$, amputation status $(p=0.760)$, or the interaction of the two $(p=0.959)$. The lateral compartment indicated that there were significant differences in exercise type among the groups present, so those values were analyzed with a post-hoc Tukey pairwise comparison. The results of this comparison are shown in Table 3.7.

Table 3.7 Lateral post-hoc Tukey pairwise comparison results ( ${ }^{*}$ indicates a significant result, $p<0.05$ )

\begin{tabular}{c|c} 
Interaction & P-Value \\
\hline Amputee gait - Amputee cycling & $0.00012^{*}$ \\
Control cycling - Amputee cycling & 0.997 \\
Control gait - Amputee cycling & $0.00013^{*}$ \\
Control cycling - Amputee gait & $0.00018^{*}$ \\
Control gait - Amputee gait & 1.000 \\
Control gait - Control cycling & 0.00020 *
\end{tabular}

The Tukey results support the conclusions of the ANOVA test. There is a significant difference in tibial contact pressure due to exercise type when looking at similar subjects ( $p=0.00012$ for amputees and $p=0.00020$ for controls) as well as between subject types $(p=0.00013$ for control gait - amputee cycling and $p=0.00018$ for control cycling - amputee gait). There is no difference based on amputation status in either gait $(p=1.000)$ or cycling $(p=0.997)$. 


\section{DISCUSSION}

\subsection{Subject Contact Stress}

\subsubsection{FEA Results}

As stated previously, there were no significant trends in the medial side while only exercise type was significant in the lateral side. This suggests that an individual, regardless of amputation status, would be at less risk for lateral OA with cycling as compared to gait. Additionally, there would be no change in their medial OA risk. One of the larger differences between the gait and cycling cases was that the cycling peak load occurred at a much higher flexion angle, on average 19.83 degrees in gait versus 66.05 degrees in cycling. As the femur rotated through its range of motion, the femur shifted posteriorly and the ligaments extended slightly, causing them to apply a larger load, particularly in the MCL. This larger ligament load caused a higher medial stress. While the applied compressive load was $91.9 \%$ lower in cycling than gait, this higher stress due to ligament tension caused the net contact stress to only be reduced by $25.1 \%$ between gait and cycling (due to the standard deviations of $44.4 \%$ in gait and $18.0 \%$ in cycling, this $25.1 \%$ difference in means was not significant). The opposite trend happened in the LCL. The LCL inserts into the superior aspect of the fibula, which is located slightly posterior to the tibial plateau. Therefore, as the femur rotated, the strain in the LCL decreased. This combined with the lower applied loads to cause a lower lateral contact stress compared to gait. 


\subsubsection{Experimental Comparison}

Other kinematic studies have found that cycling produces significantly lower resultant forces and rotation moments than gait [14], [15] which should cause lower contact pressures. This supports the finding of this study that cycling reduces the risk for lateral OA. However, the applied force and moment resultant are only a component of the overall cartilage loading, which is highly dependent on the ligament forces. The action of the collateral ligaments at high flexion angles could explain why there was no significant medial contact pressure difference between gait and cycling. This also leaves the model open to errors if the ligaments are incorrectly defined. Additionally, cohort studies have shown that amputees are at higher risk for OA, particularly in the medial side [4], [7], [63], [64]. If contact pressure is a highly correlated risk factor for OA, then it would be expected that amputees would exhibit higher contact stresses than the controls. However that is not what the results show, which is that contact stress is largely independent of amputation status. This is one of the shortcomings of using a generic FEA model because it is unable to reflect any underlying physiological changes that may have occurred in the amputees that leads to the higher OA incidence. Subject-specific modeling or producing a generic amputee model would yield greater insight into this phenomenon.

Kinematic studies have also shown that while TT amputees exhibit significantly different gait kinematics compared to control subjects, they develop similar resultant forces and rotation moments [15], [65]. This is consistent with the result of this study that there was no contact stress difference between TT 
amputees or controls. However, if this is the case, it may not be sufficient to predict OA risk factors solely through varying loads. If the loads are not different due to amputation status, there must be some other underlying biomechanical change that TT amputees face that results in higher OA risk. Cadaver studies have shown that ACL forces vary quite heavily on both flexion angle and applied loading [66]. This was similarly seen in the FEA models. The ligament spring elements would apply force in accordance with their relative displacement, which could change significantly as the knee articulated. Subsequently, a significant portion of the contact stress developed as a response to the ligament forces. It is highly likely that the ligaments have a significant impact on TT amputee OA risk factors. Subject-specific modeling of the ligaments would help distinguish differences in the amputee population relative to the controls.

Looking at the contact area could also give further insight into the effects of altered knee loading. Specifically, seeing how the location of maximum contact stress changes in controls versus amputees and gait versus cycling. It is not immediately clear in what way the effect could be quantified. The underlying relation to $\mathrm{OA}$ is that even if the magnitudes of maximum contact stress are not significantly different, applying the stress to a region of cartilage that does not normally see such high stress can cause accelerated degradation. Understanding how the region of maximum stress moves could be equally important to understanding OA risk as predicting the stress magnitude itself. As the loading cases in this thesis were all conducted on the same geometry, a 
single contour plot showing how the location of maximum stress moved could easily be developed.

\subsubsection{Development Procedure}

The modeling environment set up for this study is conducive to development of additional models. While the segmentation process is largely manual and tedious, it does not require a large labor investment before MRIs can be accurately segmented. Additionally, the number of persons segmenting should be minimized to reduce inter-observer error. MeshLab does not require a large amount of computational time and can efficiently process the mesh files to yield smooth surfaces. Once a target number of surface faces is developed for a given body, MeshLab can quickly produce surfaces with the target. There is that capability to automate MeshLab to run a set routine of filters to a large number of bodies, but that may not be the best approach for this situation. The highly irregular regions that randomly occurred during IP could not be anticipated and required manual adjustment of the filter parameters. The IP remeshing parameter also had to be manually adjusted to reach the target number of faces. Luckily, the filters used to create the models did not require a large amount of user time to execute. Utilizing TetGen saves large amounts of time in the development of computational meshes. Once the surface meshes are created, the same MATLAB routine can be called to create any number of models. Utilizing the ABAQUS GUI for definition of contact surfaces was again a highly manual process, however an individual familiar with the geometry and physiology can 
efficiently define all the requisite surfaces. After the geometry and contact surfaces have been separated into the correct text files, the MATLAB script can again be invoked to rapidly create and submit ABAQUS jobs with different models and loading conditions. While there are still areas of the process that can be improved or further automated (MeshLab, text file writing, output processing), the procedure outlined is better suited towards subject-specific models than previous methods.

\subsection{Model Limitations}

While the model was sufficiently converged and validated to some experimental data, it still is only a model and faces some limitations due to the assumptions made.

\subsubsection{Material Model}

One of the larger assumptions made was in how the cartilage and menisci were modeled. While there is literature supporting use of linear elastic isotropic materials, material choice still impacts the stress distribution in the cartilage. For example, a cartilage material that was too stiff or too compliant would misrepresent the contact area and skew the reported contact pressure. Including a material model that is biphasic and/or viscoelastic would increase the fidelity of reported pressures. Future work for this study could include such a material model to increase the clinical relevance of the results. 
Physiologically, the meniscus serves to distribute the load across the tibial plateau [67]. Accurate modeling of the menisci would change how they distribute stress between the femoral and tibial cartilage. The menisci exhibit transverse isotropy, with the annular direction being much stiffer than the others [68]. Increasing transverse compliance of the menisci would improve coaptation at the contact surfaces, resulting in more accurate stress distribution.

\subsubsection{Medial Contact Patch}

While the lateral contact patch generally produced smooth and plausible contour plots, the medial contact patch, particularly in gait, was often entirely concentrated in a small region around the lateral aspect. This is shown in the contour plot in Figure 4.1. The standard deviations for medial results also tended to be higher than lateral results, indicating the model was not as stable in that region. This suggests the medial kinematics of the model may not be accurate. As the medial compartment has a higher incidence rate of OA than the lateral side [64], accurate medial contact kinematics are critical for having a clinically relevant model. Additionally, experimental stress contour plots of Seitz, et. al. [62] show that the contact stress is more even distributed under the menisci. In the medial compartment, exercise choice was close to being statistically significant $(p=0.088)$. A more accurate contact patch or more subjects could change the stress distribution such that one or more interactions are, in fact, significant. A power analysis indicates that for $\beta=0.20, n=21$ subjects could indicate statistical significance. It is likely that the current contact patch is an 
artifact of the segmentation procedure. Only finite resolution can be extracted from a grayscale MRI image. Distinguishing, by hand, the boundaries of anatomical bodies based on varying shades of gray is inherently an inaccurate process. Future work could be aimed at segmenting with higher fidelity to reduce the degree of inaccurate body geometry. The final segmentation should be reviewed by an orthopedic surgeon to ensure accuracy.

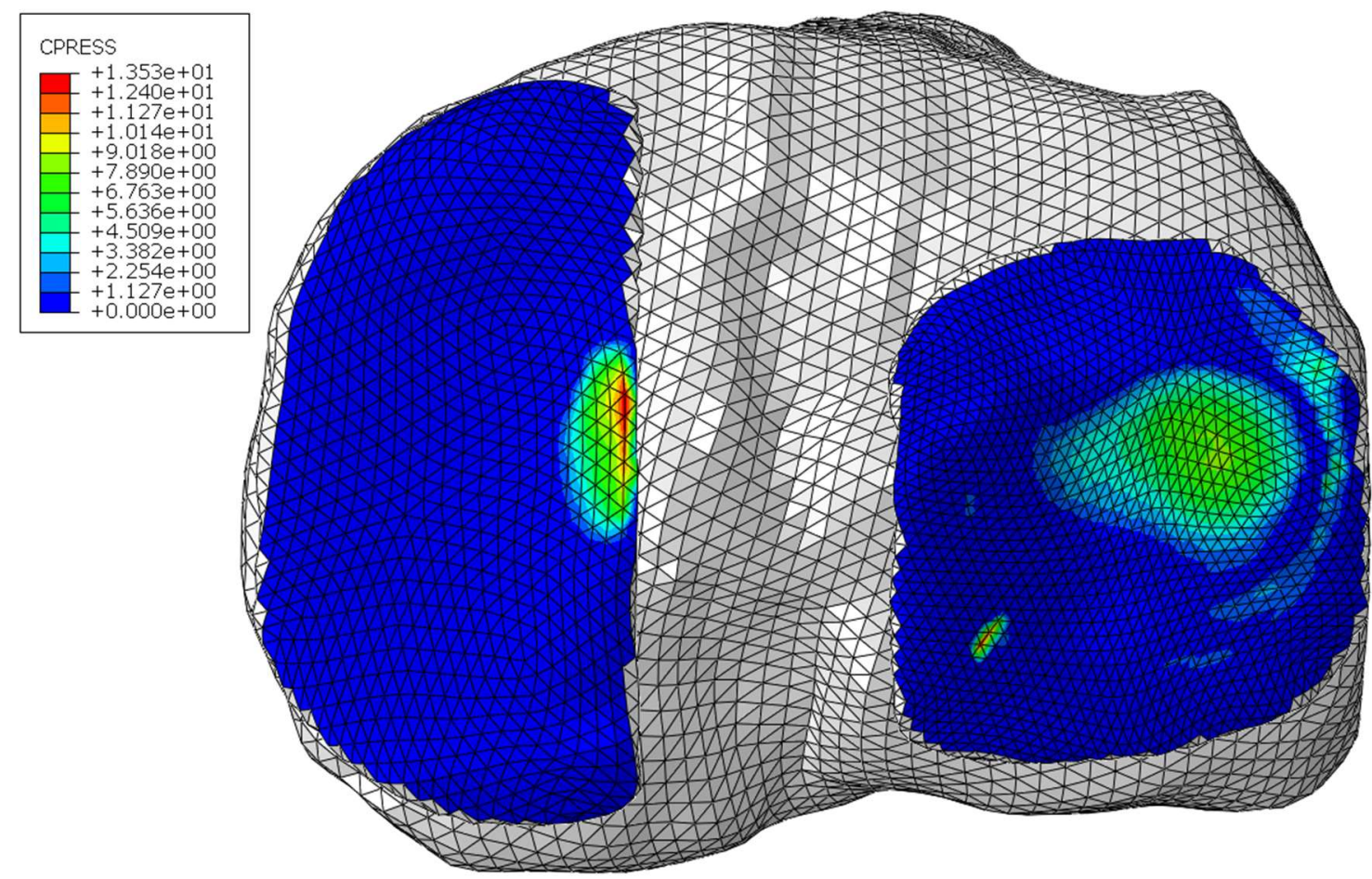

Figure 4.1 Typical gait contour plot showing poor medial contact patch.

\subsubsection{Ligament Definition}

While the ligament modeling approach used in this study is supported in literature, the model exhibited high sensitivity to the locations of the origin and insertion sites determined from MRI. Changing these locations would affect the direction of the ligaments' lines of action. Changing the direction of loads applied 
to the bones would also impact how cartilage stress developed to resist the forces. This would compound with the poor contact patch geometry and cartilage material compliance to substantially change contact pressures or cause stress concentrations. A more precise validation of the ligament attachment sites as well as their prestress definitions would create a more stable and accurate model. One such method to refine the cruciate ligament definition would be to replace the single-spring ligament bundles with multiple springs. With a singlespring definition, the line of action for the ligament is strictly directed between the two nodes chosen. This makes the ligament definition sensitive to the nodes selected, which is a subjective choice. Averaging the ligament attachments over multiple nodes reduces this direction vector sensitivity. Additionally, ligament definitions should be reviewed by an orthopedic surgeon for physiological accuracy.

\subsubsection{Joint Resultant Force}

The forces that were determined experimentally and applied to the FEA model were joint resultant forces. These consist of a force-couple system applied at the $\mathrm{KJC}$ and replicate the net force and moment developed from the joint contact force and muscle forces acting across the knee, namely the knee flexor (e.g. hamstring) and extensor (e.g. quadriceps) muscle groups. Anatomically, the TF joint can only support a compressive load normal to the tibial plateau because of the near frictionless nature of the AC. The joint shear loads and rotation moments develop because of the muscle forces that act across the joint. By only 
modeling the TF joint and neglecting interactions of the patellofemoral (PF) joint, the quadriceps force must be included in the applied load to account for its interaction. This necessitates use of the joint resultant force. To include the PF joint in the FEA model, the quadriceps component of the resultant forces would have to be removed and applied across the patella and patellar tendon to the tibia. It is valid to create a TF FEA model only if the joint resultant force is correctly determined. Including the PF joint and applying the joint resultant force would incorrectly account for the interaction of the quadriceps force. Similarly, applying just the joint contact force without the PF joint modeled would not fully describe the forces present at the knee. In this study, it was appropriate to apply the joint resultant to a model that did not include the patella. 


\section{CONCLUSIONS}

The purpose of this thesis was primarily to determine relative risk factors for TT amputees in differing exercise regimes be means of an FEA model and secondarily to develop a process by which subject-specific models could be developed. One subject's MRI was fully segmented and turned into a TF joint FEA model. The model was validated against experimental cadaveric data using linear elastic material properties for both the cartilage and menisci and non-linear springs to represent the ligaments. Six control and six TT amputee loading cases were applied to the model for each exercise mode (gait, cycling). The results indicated that all subjects, regardless of amputation status are at lower risk for lateral tibial OA with cycling than gait $(p<0.0001)$, while medial OA risk remains

unaffected. Amputees did not show higher levels of cartilage stress than controls in either exercise mode. The hypothesis of this thesis was that TT amputees would see higher cartilage contact stress because of their known higher incidence rates for OA. Additionally, cycling was expected to reduce cartilage contact stress because of the lower loads. While cycling did reduce the contact stress in the lateral compartment, it did not do so for the medial compartment which is known to be more prone to OA. Also, TT amputees did not show any differences from the control subjects. Taken together, these two results suggest that the load distribution mechanisms in the knee may be more complicated than was anticipated. Further modeling is needed to accurately characterize how the knee absorbs and distributes loads. 
This thesis also set out to establish a methodology that can be applied to create subject-specific FEA models. While there are currently some limitations to be improved upon, the underlying process can be used to develop FEA models in an expedient manner. Several aspects of mesh generation were automated using MATLAB scripts. This constituted a bulk of the work in this thesis. Along the way, several modeling steps were made with the specific aim of helping facilitate a more automated model development process. In the future, these can be expanded to provide greater functionality. With continued improvement, the procedure outlined in this thesis can be used to conduct extensive subjectspecific FEA studies for novel research aims. 


\section{REFERENCES}

[1] R. Altman, E. Asch, D. Bloch, G. Bole, D. Borenstein, K. Brandt, W.

Christy, T. D. Cooke, R. Greenwald, M. Hochberg, D. Howell, D.

Kaplan, W. Koopman, S. Longley, H. Mankin, D. J. McShane, T.

Medsger, R. Meenan, W. Mikkelsen, R. Moskowitz, W. Murphy, B.

Rothschild, M. Segal, L. Sokoloff, and F. Wolfe, "Development of

criteria for the classification and reporting of osteoarthritis:

Classification of osteoarthritis of the knee," Arthritis Rheum., vol.

29, no. 8, pp. 1039-1049, 1986.

[2] D. J. Hunter, D. Schofield, and E. Callander, "The individual and socioeconomic impact of osteoarthritis," Nat. Rev. Rheumatol., vol. 10, no. 7, pp. 437-441, Jul. 2014.

[3] M. G. Cisternas, L. Murphy, J. J. Sacks, D. H. Solomon, D. J. Pasta, and C. G. Helmick, "Alternative Methods for Defining Osteoarthritis and the Impact on Estimating Prevalence in a US Population-Based Survey," Arthritis Care Res., vol. 68, no. 5, pp. 574-580, 2016.

[4] D. C. Norvell, J. M. Czerniecki, G. E. Reiber, C. Maynard, J. A. Pecoraro, and N. S. Weiss, "The prevalence of knee pain and symptomatic knee osteoarthritis among verteran traumatic amputees and nonamputees," Arch Phy Med Rehabil, vol. 86, no. 3, pp. 487-493, 2005.

[5] K. Bialik, "The changing face of America's veteran population," Pew Research Center, 2017. [Online]. Available: 
http://www.pewresearch.org/fact-tank/2017/11/10/the-changingface-of-americas-veteran-population/. [Accessed: 27-May-2018].

[6] Department of Veterans Affairs "VA 2017 Budget Request: Fast Facts," 2017.

[7] M. H. Ebrahimzadeh and S. Hariri, "Long-term outcomes of unilateral transtibial amputations," Mil. Med., vol. 174, no. 6, pp. 593-597, 2009.

[8] R. Castanharo, B. S. Da Luz, A. C. Bitar, C. O. D’Elia, W. Castropil, and M. Duarte, "Males still have limb asymmetries in multijoint movement tasks more than 2 years following anterior cruciate ligament reconstruction," J. Orthop. Sci., vol. 16, no. 5, pp. 531535, 2011.

[9] T. P. Andriacchi and C. O. Dyrby, "Interactions between kinematics and loading during walking for the normal and ACL deficient knee," $J$. Biomech., 2005.

[10] A. K. Silverman, N. P. Fey, A. Portillo, J. G. Walden, G. Bosker, and R. R. Neptune, "Compensatory mechanisms in below-knee amputee gait in response to increasing steady-state walking speeds," Gait Posture, vol. 28, no. 4, pp. 602-609, 2008.

[11] T. D. Royer and C. A. Wasilewski, "Hip and knee frontal plane moments in persons with unilateral, trans-tibial amputation," Gait Posture, vol. 23, no. 3, pp. 303-306, 2006.

[12] J. A. Buckwalter, D. D. Anderson, T. D. Brown, Y. Tochigi, and J. A. 
Martin, "The Roles of Mechanical Stresses in the Pathogenesis of Osteoarthritis-Implications for Treatment of Joint Injuries," Cartilage, pp. 1-9, 2013.

[13] T. P. Andriacchi, S. Koo, and S. F. Scanlan, "Gait mechanics influence healthy cartilage morphology and osteoarthritis of the knee," $\mathrm{J}$. Bone Jt. Surg. - Ser. A, vol. 91, no. SUPPL. 1, pp. 95-101, 2009.

[14] M. V. Pottinger, K. Mavrommati, S. J. Hazelwood, and S. M. Klisch, "EMGDriven Inverse Dynamic Analysis of Knee Contact Forces During Gait and Cycling Using OpenSim," in Proceedings of the 2017 Summer Biomechanics, Bioengineering, and Biotransport Conference, 2017.

[15] E. A. Heyde, G. Orekhov, A. M. Robinson, S. J. Hazelwood, and S. M. Klisch, "Hip and knee forces for transtibial amputees in gait and cycling," in 42nd Annual Meeting of the American Society of Biomechanics, 2018.

[16] K. M. Moerman, C. A. Holt, S. L. Evans, C. K. Simms, and K. M. Moerman, "Digital image correlation and finite element modelling as a method to determine mechanical properties of human soft tissue in vivo."

[17] L. Li, S. Patil, N. Steklov, W. Bae, D. DD, R. Sah, and B. J. Freg, "Predicting In Vitro Articular Cartilage Wear in the Patellofemoral Joint using Finite Element Modeling," in 36th Annual Meeting of the American Society of Biomechanics, 2012. 
[18] B. Jones, C. T. Hung, and G. Ateshian, "Biphasic Analysis of Cartilage Stresses in the Patellofemoral Joint," J. Knee Surg., pp. 92-98, 2015.

[19] M. Taffetani, M. Griebel, D. Gastaldi, S. M. Klisch, and P. Vena, "Poroviscoelastic finite element model including continuous fiber distribution for the simulation of nanoindentation tests on articular cartilage," J. Mech. Behav. Biomed. Mater., vol. 32, pp. 17-30, 2014.

[20] K. S. Halonen, M. E. Mononen, J. S. Jurvelin, J. T??yr??s, and R. K. Korhonen, "Importance of depth-wise distribution of collagen and proteoglycans in articular cartilage-A 3D finite element study of stresses and strains in human knee joint," J. Biomech., 2013.

[21] M. E. Mononen, M. T. Mikkola, P. Julkunen, R. Ojala, M. T. Nieminen, J. S. Jurvelin, and R. K. Korhonen, "Effect of superficial collagen patterns and fibrillation of femoral articular cartilage on knee joint mechanics-A 3D finite element analysis," J. Biomech., 2012.

[22] D. C. Fithian, M. a Kelly, and V. C. Mow, "Material properties and structure-function relationships in the menisci.," Clin. Orthop. Relat. Res., pp. 19-31, 1990.

[23] E. Peña, B. Calvo, M. A. Martínez, D. Palanca, and M. Doblaré, "Finite element analysis of the effect of meniscal tears and meniscectomies on human knee biomechanics," Clin. Biomech., vol. 20, no. 5, pp. 498-507, Jun. 2005. 
[24] P. Łuczkiewicz, K. Daszkiewicz, W. Witkowski, J. Chróścielewski, and W. Zarzycki, "Influence of meniscus shape in the cross sectional plane on the knee contact mechanics," J. Biomech., 2015.

[25] J. R. Meakin, N. G. Shrive, C. B. Frank, and D. A. Hart, "Finite element analysis of the meniscus: The influence of geometry and material properties on its behaviour," Knee, vol. 10, no. 1, pp. 33-41, 2003.

[26] M. Adouni, A. Shirazi-Adl, and R. Shirazi, "Computational biodynamics of human knee joint in gait: From muscle forces to cartilage stresses," J. Biomech., 2012.

[27] C. D. Untaroiu, N. Yue, and J. Shin, "A finite element model of the lower limb for simulating automotive impacts," Ann. Biomed. Eng., vol. 41, no. 3, pp. 513-526, 2013.

[28] E. F. Morgan, H. H. Bayraktar, and T. M. Keaveny, "Trabecular bone modulus-density relationships depend on anatomic site," J. Biomech., 2003.

[29] L. Blankevoort, J. H. Kuiper, R. Huiskes, and H. J. Grootenboer, "Articular contact in a three-dimensional model of the knee," J. Biomech., vol. 24, no. 11, pp. 1019-1031, 1991.

[30] G. A. Johnson, B. A. Smith, and S. Woo, "Mathematical Modeling of Ligaments and Tendons," J. Biomech. Eng., vol. 115, pp. 468-473, 1993.

[31] J. A. Weiss, J. C. Gardiner, B. J. Ellis, T. J. Lujan, and N. S. Phatak, "Three-dimensional finite element modeling of ligaments: Technical 
aspects," Med. Eng. Phys., vol. 27, pp. 845-861, 2005.

[32] F. Galbusera, M. Freutel, L. Dürselen, M. D'Aiuto, D. Croce, T. Villa, V.

Sansone, and B. Innocenti, "Material models and properties in the finite element analysis of knee ligaments: a literature review.,"

Front. Bioeng. Biotechnol., vol. 2, no. November, p. 54, 2014.

[33] A. Erdemir, "A Finite Element Analysis Workflow in Biomechanics,"

Cleaveland, OH, 2015.

[34] T. F. Besier, G. E. Gold, G. S. Beaupré, and S. L. Delp, "A modeling framework to estimate patellofemoral joint cartilage stress in vivo," in Medicine and Science in Sports and Exercise, 2005.

[35] J. J. Elias and A. J. Cosgarea, "Computational Modeling: An Alternative Approach for Investigating Patellofemoral Mechanics," Sports Med. Arthrosc., vol. 15, no. 2, pp. 89-94, 2007.

[36] A. A. Ali, S. S. Shalhoub, A. J. Cyr, C. K. Fitzpatrick, L. P. Maletsky, P. J. Rullkoetter, and K. B. Shelburne, "Validation of predicted patellofemoral mechanics in a finite element model of the healthy and cruciate-deficient knee," J. Biomech., 2016.

[37] L. Li, S. Patil, N. Steklov, W. Bae, D. D. D’Lima, R. L. Sah, and B. J. Fregly, "Predicting In Vitro Articular Cartilage Wear In The Patellofemoral Joint Using Finite Element Modeling," in 36th Annual Meeting of the American Society of Biomechanics, 2012, vol. 186.

[38] M. E. Mononen, J. S. Jurvelin, and R. K. Korhonen, "Effects of radial tears and partial meniscectomy of lateral meniscus on the knee joint 
mechanics during the stance phase of the gait cycle - A 3D finite element study," J. Orthop. Res., 2013.

[39] T. P. Andriacchi, P. L. Briant, S. L. Bevill, and S. Koo, "Rotational Changes at the Knee after ACL Injury Cause Cartilage Thinning," Clin. Orthop. Relat. Res., vol. 442, pp. 39-44, 2006.

[40] J. Suggs, C. Wang, and G. Li, "The effect of graft stiffness on knee joint biomechanics after ACL reconstruction - A 3D computational simulation," Clin. Biomech., 2003.

[41] G. Papaioannou, C. K. Demetropoulos, and Y. H. King, "Predicting the effects of knee focal articular surface injury with a patient-specific finite element model," Knee, 2010.

[42] S. Pal, J. E. Langenderfer, J. Q. Stowe, P. J. Laz, A. J. Petrella, and P. J. Rullkoetter, "Probabilistic modeling of knee muscle moment arms: Effects of methods, origin-insertion, and kinematic variability," Ann. Biomed. Eng., 2007.

[43] M. A. Baldwin, P. J. Laz, J. Q. Stowe, and P. J. Rullkoetter, "Efficient probabilistic representation of tibiofemoral soft tissue constraint," Comput. Methods Biomech. Biomed. Engin., 2009.

[44] C. K. Fitzpatrick, M. A. Baldwin, and P. J. Rullkoetter, "Computationally Efficient Finite Element Evaluation of Natural Patellofemoral Mechanics."

[45] S. Wangerin, "Development and Validation of a Human Knee Joint Finite Element Model for Tissue Stress and Strian Predictions During 
Exercise," no. December, p. 85, 2013.

[46] M. K. Sylvia, "Development of a Human Tibiofemoral Joint Finite Element Model to Investigate the Effects of Obesity and Malalignment on Joint Contact Pressure," California Polytechnic State University, 2015.

[47] N. A. Czapla, "Human Knee Joint Finite Element Model Using a Two Bundle Anterior Cruciate Ligament : Validation and Gait Analysis," California Polytechnic State University, 2015.

[48] P. A. Yushkevich, J. Piven, H. C. Hazlett, R. G. Smith, S. Ho, J. C. Gee, and G. Gerig, "User-guided 3D active contour segmentation of anatomical structures: Significantly improved efficiency and reliability," Neuroimage, vol. 31, no. 3, pp. 1116-1128, 2006.

[49] H. Si, "TetGen, a Quality Tetrahedral Mesh Generator," AMC Trans. Math. Softw., vol. 41, no. 2, p. 11, 2015.

[50] P. Cignoni, M. Callieri, M. Corsini, M. Dellepiane, F. Ganovelli, and G. Ranzuglia, "MeshLab: An Open-Source Mesh Processing Tool," in Sixth Eurographics Italian Chapter Conference, 2008, pp. 129-136.

[51] G. Taubin, "Curve and surface smoothing without shrinkage," Proceedings of IEEE International Conference on Computer Vision. pp. 852857, 1995.

[52] G. Taubin, T. Zhang, and G. Golub, "Optimal Surface Smoothing as Filter Design," Comput. Vision-ECCV'96, vol. 20404, no. August, pp. 283-292, 1996. 
[53] N. Pietroni, M. Tarini, and P. Cignoni, "Almost isometric mesh parameterization through abstract domains," IEEE Trans. Vis. Comput. Graph., 2010.

[54] S. A. Maas, B. J. Ellis, D. S. Rawlins, and J. A. Weiss, "Finite element simulation of articular contact mechanics with quadratic tetrahedral elements," J. Biomech., vol. 49, no. 5, pp. 659-667, 2016.

[55] K. M. Moerman and T. G. Badger, "Gibboncode/Gibbon: Gibbon: The Geometry And Image-Based Bioengineering Add-On (Release: Hylobates Albibarbis)," doi.org, p., Sep. 2017.

[56] M. Kazemi, L. P. Li, M. D. Buschmann, and P. Savard, "Partial Meniscectomy Changes Fluid Pressurization in Articular Cartilage in Human Knees," J. Biomech. Eng., vol. 134, 2012.

[57] T. L. Haut Donahue, M. L. Hull, M. M. Rashid, C. R. Jacobs, T. L. H. Donahue, M. L. Hull, M. M. Rashid, and C. R. Jacobs, "A Finite Element Model of the Human Knee Joint for the Study of TibioFemoral Contact," J. Biomech. Eng., vol. 124, no. 3, p. 273, 2002.

[58] E. Pena, B. Calvo, M. A. Martinez, and M. Doblare, "A three-dimensional finite element analysis of the combined behavior of ligaments and menisci in the healthy human knee joint," J. Biomech., vol. 39, no. 9, pp. 1686-1701, 2006.

[59] L. Blankevoort and R. Huiskes, "Validation of a three-dimensional model of the knee," J. Biomech., vol. 29, no. 7, pp. 955-961, 1996.

[60] G. Li, J. Gil, A. Kanamori, and S.-Y. S. L. Y. Woo, "A validated three- 
dimensional computational model of a human knee joint," $J$.

Biomech. Eng., vol. 121, no. 6, pp. 657-662, 1999.

[61] R. Shirazi and A. Shirazi-Adl, "Analysis of partial meniscectomy and ACL reconstruction in knee joint biomechanics under a combined loading," Clin. Biomech., 2009.

[62] A. M. Seitz, A. Lubomierski, B. Friemert, A. Ignatius, and L. Dürselen, "Effect of partial meniscectomy at the medial posterior horn on tibiofemoral contact mechanics and meniscal hoop strains in human knees," J. Orthop. Res., 2012.

[63] C. B. Robbins, D. J. Vreeman, M. S. Sothmann, S. L. Wilson, and N. B. Oldridge, "A review of the long-term health outcomes associated with war-related amputation," Mil. Med., vol. 174, no. 6, pp. 588592, 2009.

[64] A. J. Baliunas, D. E. Hurwitz, A. B. Ryals, A. Karrar, J. P. Case, J. A. Block, and T. P. Andriacchi, "Increased knee joint loads during walking are present in subjects with knee osteoarthritis,"

Osteoarthr. Cartil., vol. 10, no. 7, pp. 573-579, 2002.

[65] C. M. Powers, S. Rao, and J. Perry, "Knee kinetics in trans-tibial amputee gait," Gait Posture, vol. 8, no. 1, pp. 1-7, 1998.

[66] K. L. Markolf, D. M. Burchfield, M. M. Shapiro, M. F. Shepard, G. A. M. Finerman, and J. L. Slauterbeck, "Combined knee loading states that generate high anterior cruciate ligament forces," J. Orthop. Res., 1995. 
[67] I. L. Paul, E. L. Radin, and R. M. Rose, "Role of Mechanical Factors In Pathogenesis of Primary Osteoarthritis," Lancet, pp. 519-522, 1972.

[68] M. Freutel, H. Schmidt, L. Dürselen, A. Ignatius, and F. Galbusera, "Finite element modeling of soft tissues: Material models, tissue interaction and challenges," Clinical Biomechanics. 2014. 


\section{APPENDIX A: GAIT AND CYCLING CONTOUR PLOTS}

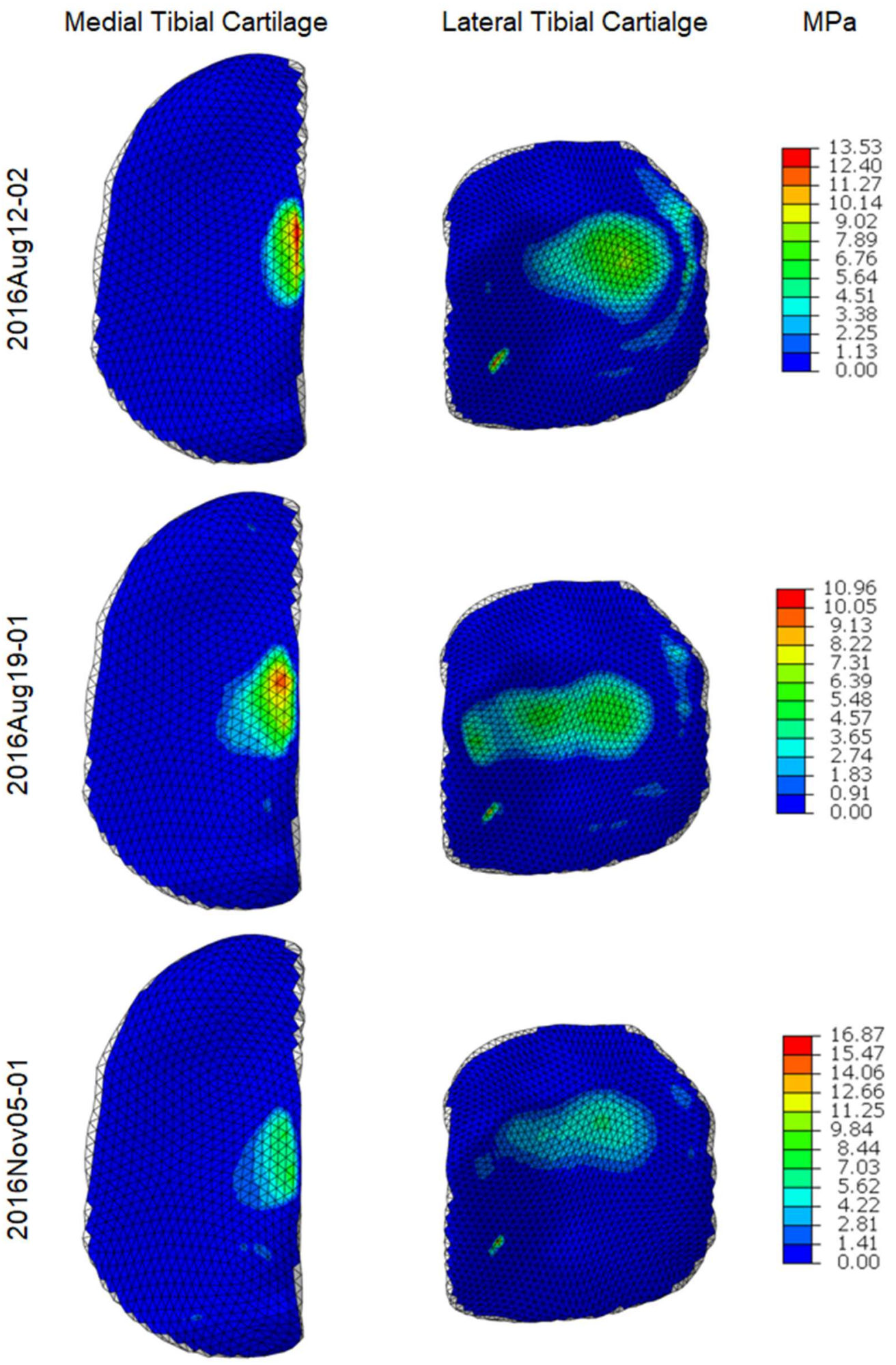

Figure A.1 Control subject contour plots in gait. 

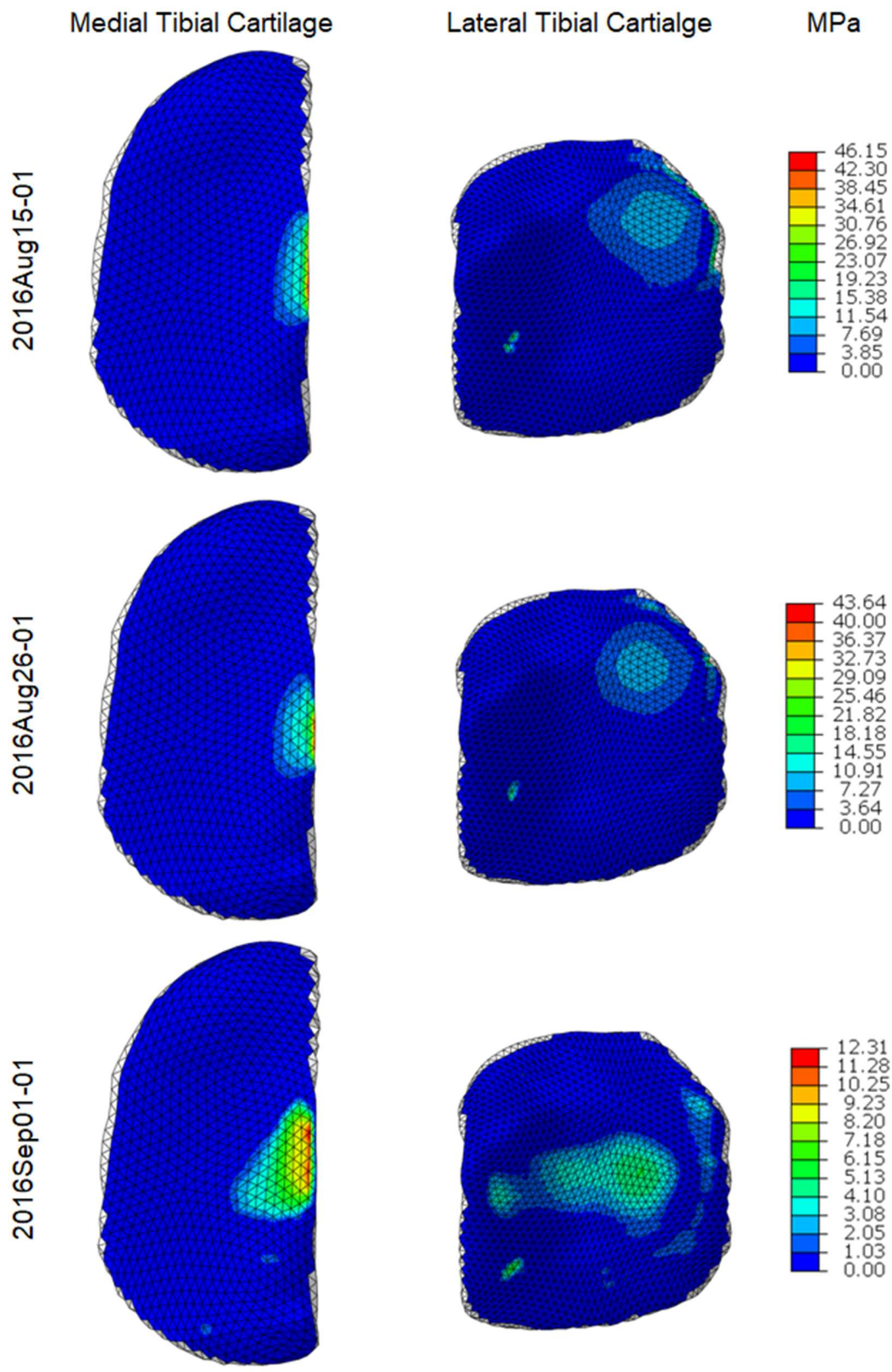

Figure A.2 Control subject contour plots in gait (cont.). 


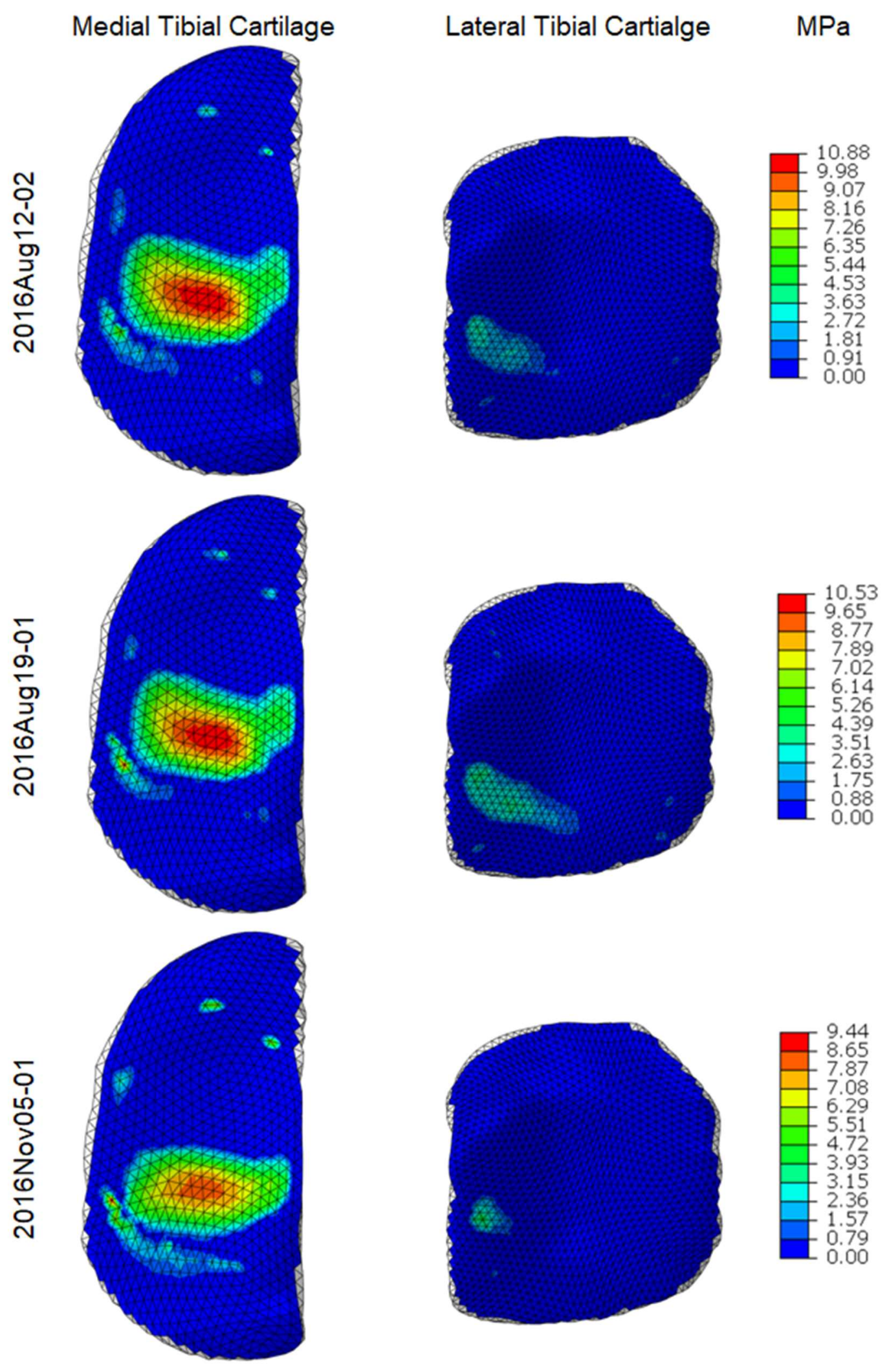

Figure A.3 Control subject contour plots in cycling. 


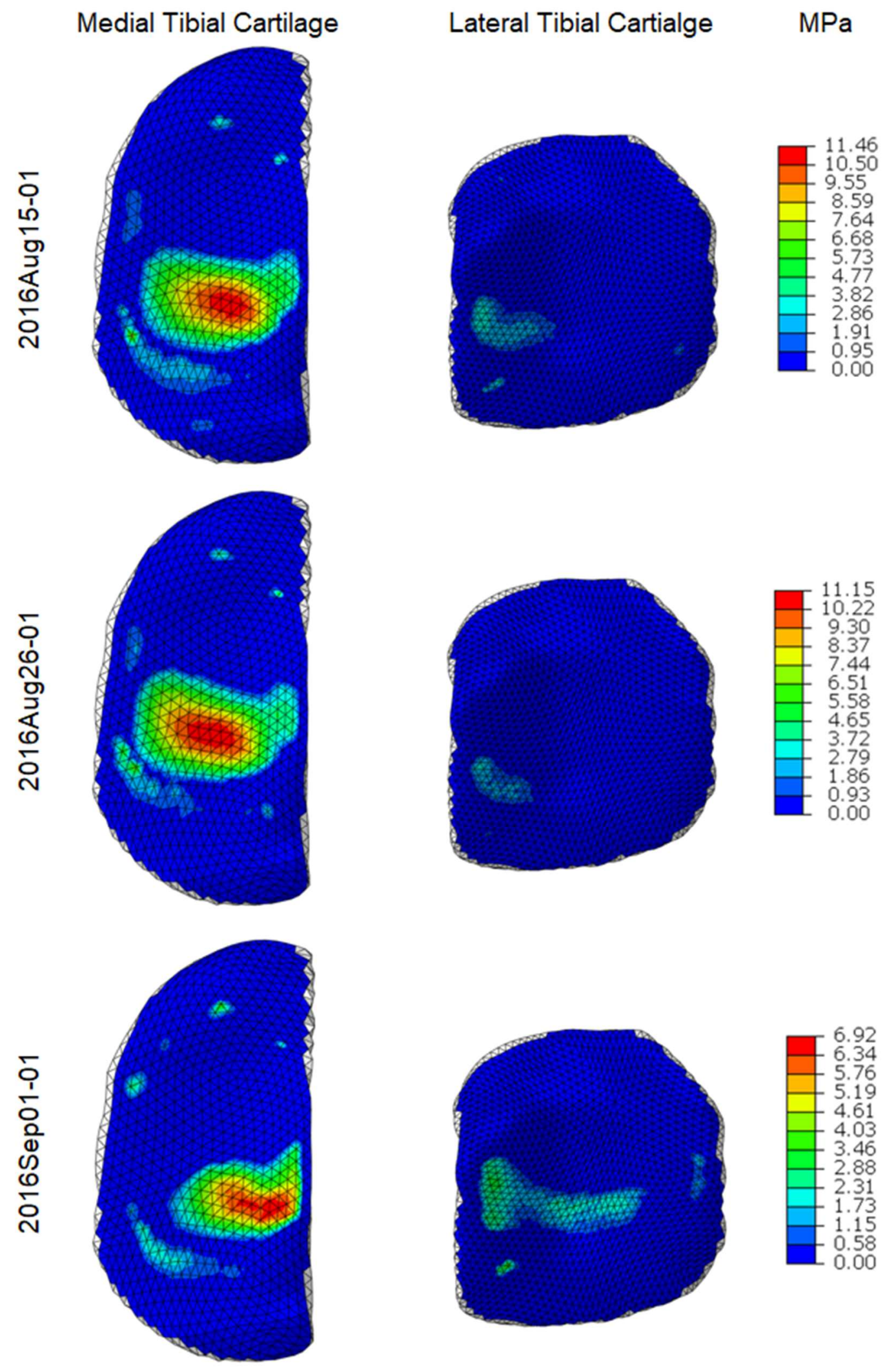

Figure A.4 Control subject contour plots in cycling (cont.). 


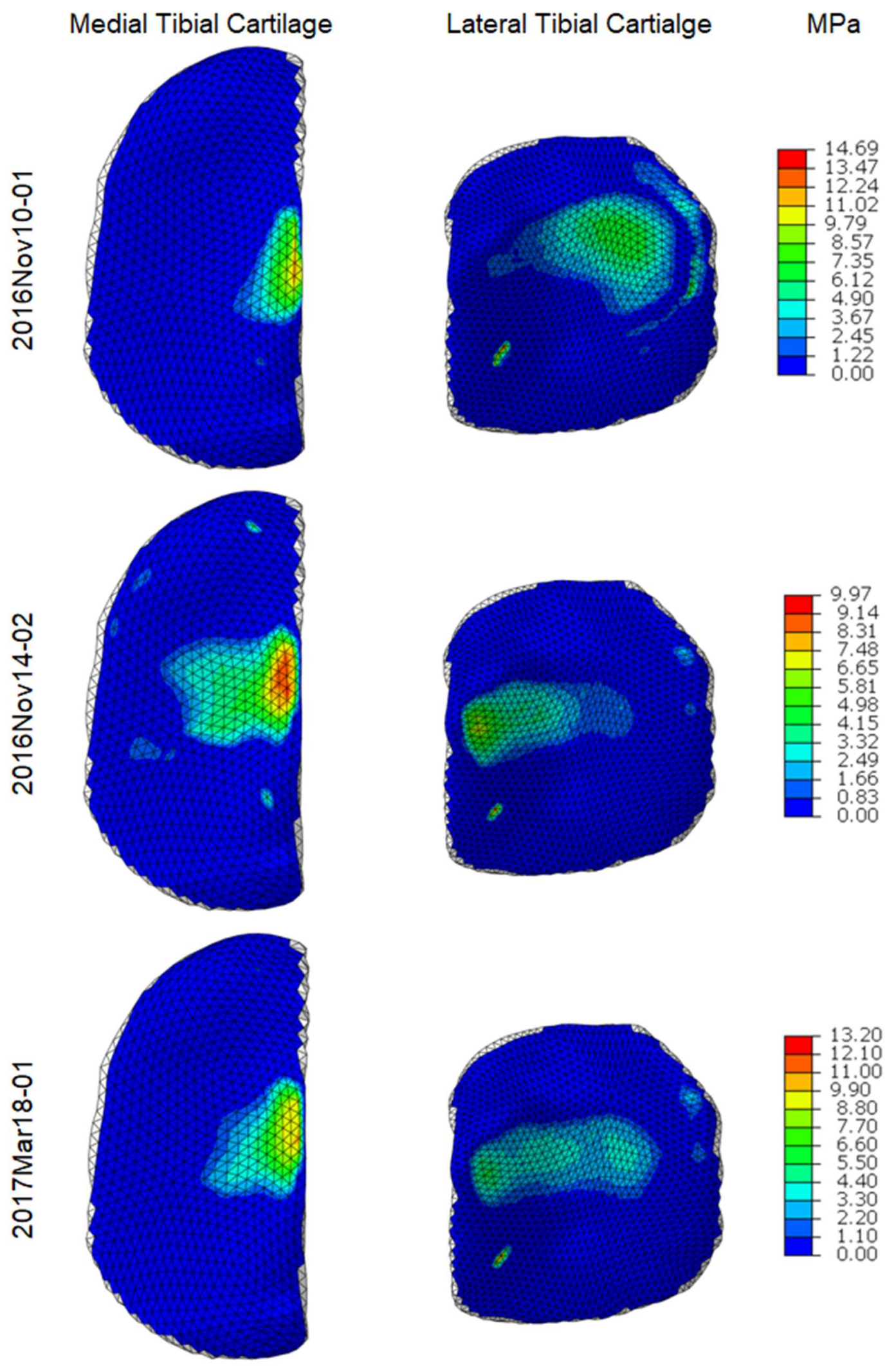

Figure A.5 Amputee subject contour plots in gait. 

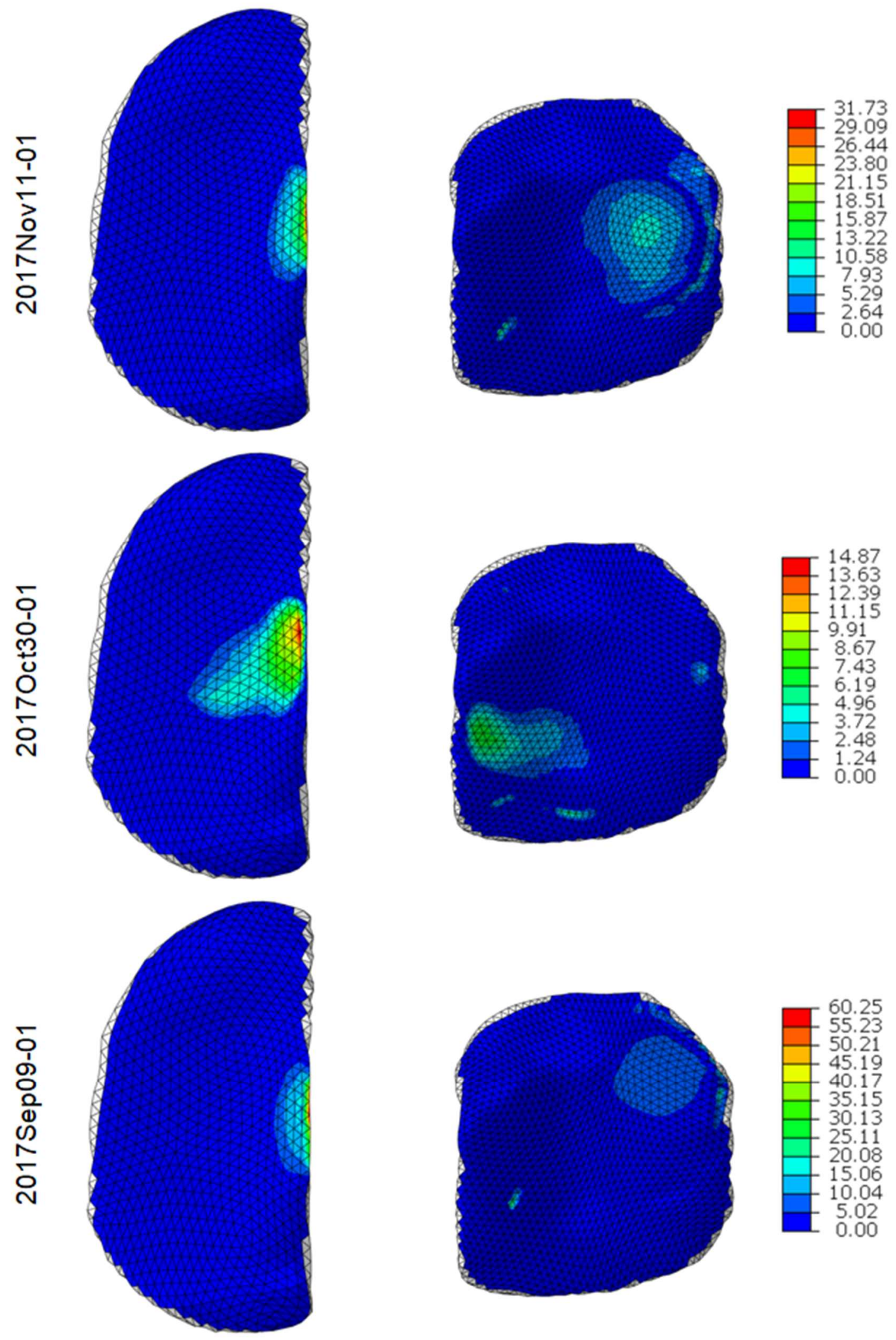

Figure A.6 Amputee contour plots in gait (cont.). 


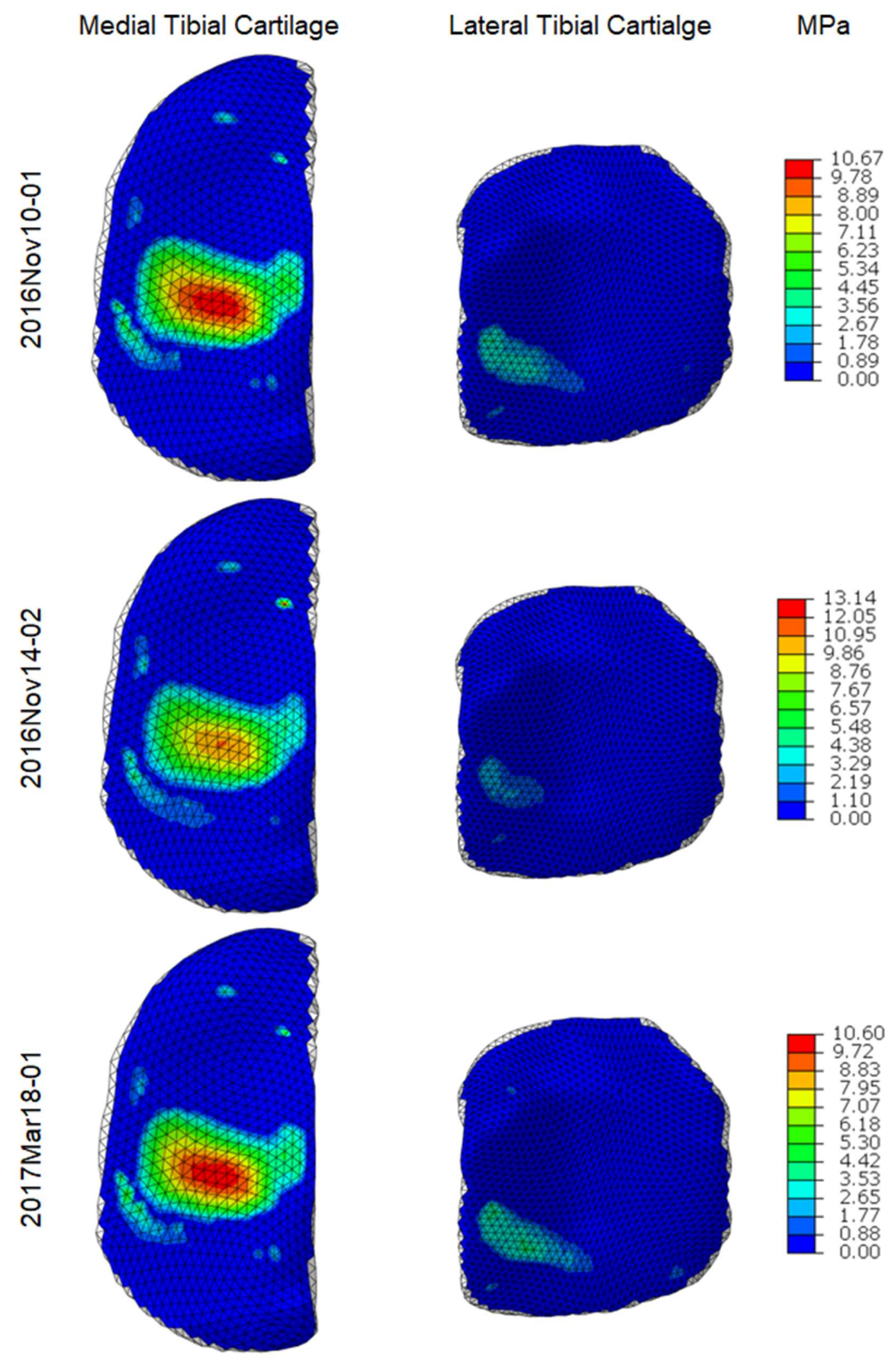

Figure A.7 Amputee subject contour plots in cycling. 

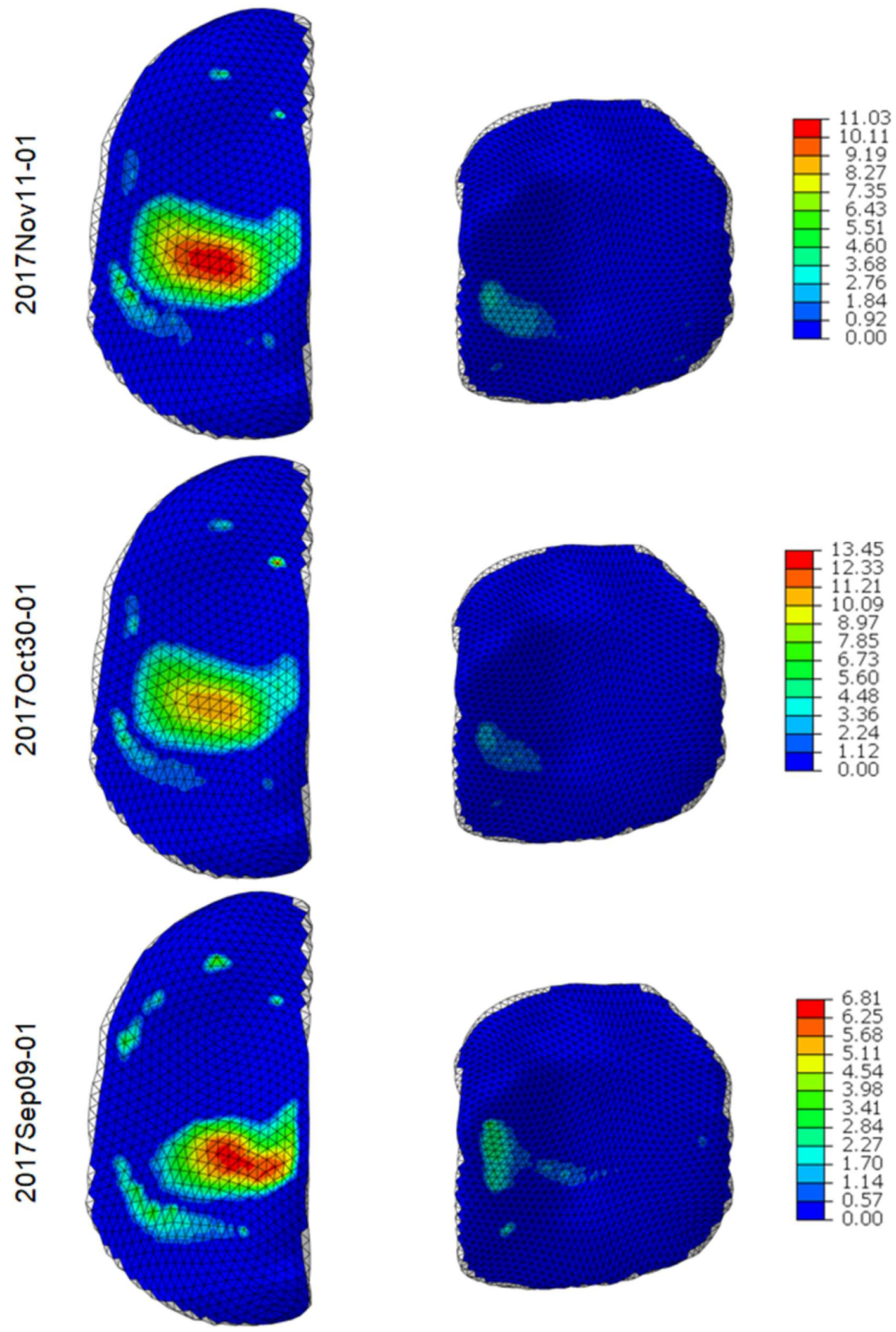

Figure A.8 Amputee subject contour plots in cycling (cont.). 